\title{
Moving Target Search with Environment Abstraction
}

\author{
By \\ Alper Yörükçü \\ A thesis submitted to \\ the Faculty of Graduate Studies and Research \\ in partial fulfilment of \\ the requirements for the degree of \\ Master of Computer Science \\ Ottawa-Carleton Institute for Computer Science \\ School of Computer Science \\ Carleton University \\ Ottawa, Ontario
}

April 17, 2015

(C) Copyright

2015, Alper Yörükçü 
The undersigned hereby recommend to the Faculty of Graduate Studies and Research acceptance of the thesis,

Moving Target Search with Environment Abstraction

Submitted by

Alper Yörükçü

\begin{tabular}{c} 
Doug Howe \\
(Director, School of Computer Science) \\
\hline Doron Nussbaum \\
(Thesis Supervisor)
\end{tabular}

Carleton University

April 17, 2015 


\section{Abstract}

Moving Target Search (MTS) is a dynamic path planning problem, where an agent is trying to reach a moving entity with a minimum path cost. Problems of this nature can be found in video games and dynamic robotics, which require fast processing time (real time). In this thesis, we introduce a new algorithm for this problem - the Moving Target Search with Subgoal Graphs (MTSub). MTSub is based on environment abstraction and uses Subgoal Graphs to speed up searches without giving up cost minimal paths. The algorithm is optimal with respect to the knowledge that the agent has during the search. Experimental results show that MTSub meets the requirement of real time performance (e.g., 5 microseconds per step). Compared to G-FRA*, which is the best known dynamic algorithm so far, MTSub is up to 29 times faster in average time per step, and 186 times faster in maximum time per step. MTSub also compares fairly well against MtsCopa, which is an algorithm that stores all possible paths in preprocessing and return them in run time. Although in this case MTSub is up to 3.89 times slower in average response time and up to 6.81 times slower in maximum response time, it performed much better than MtsCopa in the processing phase up to 220,000 times faster and requiring up to 44 times less space. According to our experiments in four different map domains with two target movement strategies, MTSub establishes itself as the new state-of-the-art in optimal Moving Target Search without heavy weight preprocessing. 


\section{Acknowledgements}

First, I would like to thank my advisor Doron Nussbaum for his guidance and support. I would also like to thank my thesis committee Tony White, Mark Lanthier and Amiya Nayak for their constructive comments. I thank Sven Koenig, Tansel Uras and Xiaoxun Sun for providing their source code. I would also like to thank Joshua Beltramin, Fraser MacQuarrie and Seda Gunduz for proofreading. Last, I would like to thank my family. 


\section{Contents}

Abstract

Acknowledgements $\quad$ iv

1 Introduction $\quad 1$

1.1 Contribution Summary . . . . . . . . . . . . . . . 3

1.2 Thesis organization $\ldots \ldots \ldots \ldots \ldots \ldots \ldots \ldots$

2 Background $\quad 5$

2.1 Search Space Representation . . . . . . . . . . . . . . . 6

2.1 .1 Grids . . . . . . . . . . . . . . . . . 6

2.1 .2 Navigation Meshes . . . . . . . . . . . . . . . 7

2.2 Problem Definition . . . . . . . . . . . . . . . . . . 9

2.2 .1 Optimal Solution . . . . . . . . . . . . . . . . . 9 9

2.3 Previous Work . . . . . . . . . . . . . . . . . . . 11

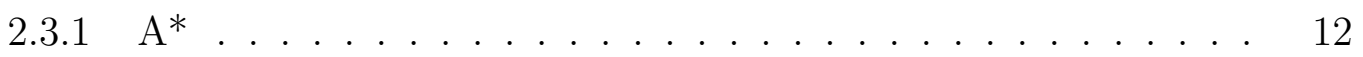

2.3.2 Generalized Fringe Retrieving $A^{*} \ldots \ldots \ldots \ldots$

2.3.3 MTS with Compressed Path Databases . . . . . . . . . . 21

2.3.4 A Generic Framework for MTS . . . . . . . . . . . . 23

2.3 .5 Subgoal Graphs . . . . . . . . . . . . . . . . . . 24

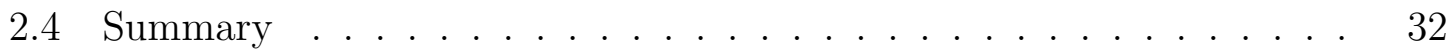

3 Moving Target Search with Subgoal Graphs 33

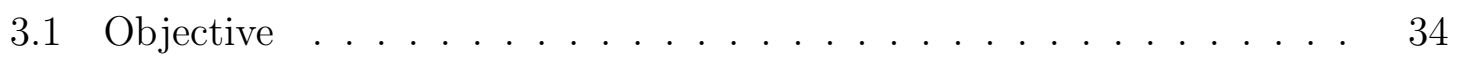


3.2 Key Idea . . . . . . . . . . . . . . . . . . . . . . . . . . . 35

3.3 Description . . . . . . . . . . . . . . . . 36

3.4 Example . . . . . . . . . . . . . . . . . . . 45

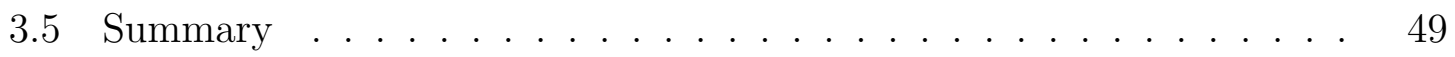

4 Experimental Results and Analysis $\quad 50$

4.1 Objective and Testing Methodology . . . . . . . . . . . . 50

4.2 The Experimental Setups . . . . . . . . . . . . . . . . . . 51

4.2 .1 Algorithm Implementations . . . . . . . . . . . . . 52

$4.2 .2 \quad$ Map Domains . . . . . . . . . . . . . . . . . . . . 52

4.2 .3 Behaviour of Targets . . . . . . . . . . . . . 56

4.2 .4 Measures . . . . . . . . . . . . . . . . . 57

4.3 Results . . . . . . . . . . . . . . . . . . . . . . 58

4.3.1 Comparison with Dynamic Algorithms for MTS . . . . . . 58

4.3.2 Comparison with MTSCopa . . . . . . . . . . . . . . 65

4.3 .3 Comparison with Extensions . . . . . . . . . . . . . . 70

4.4 Summary . . . . . . . . . . . . . . . . . . . 75

$\begin{array}{lll}5 & \text { Conclusion } & 77\end{array}$

$\begin{array}{ll}\text { Bibliography } & 80\end{array}$

$\begin{array}{ll}\text { Appendices } & 85\end{array}$

$\begin{array}{ll}\text { A Glossary } & 86\end{array}$ 


\section{List of Tables}

$2.1 \quad h$ values for the unblocked cells . . . . . . . . . . . . . 15

$2.2 \mathrm{~g}$ and $f$ values for the unblocked cells . . . . . . . . . 15

4.1 Map Class Characteristics . . . . . . . . . . . . . . . . 54

4.2 Experiments in 512x512 maps(Random Moving Target) . . . . . . . 60

4.3 Experiments in 512x512 maps(Way-point Following Target) . . . . . 62

4.4 Comparison between MtsCopa and MTSub . . . . . . . . . 65

4.5 Preprocessing Requirements of MTSub . . . . . . . . . . 68

4.6 MTSub vs. MTSub with Moving Window . . . . . . . . . 73 


\section{List of Figures}

2.1 Grid Representation . . . . . . . . . . . . . . . . . 7

2.28 Connected Grid . . . . . . . . . . . . . . . . . . . . . 8

2.3 Navigation Mesh . . . . . . . . . . . . . . . . . . . . . . . 9

2.4 Two possible paths with the same cost $\ldots \ldots \ldots \ldots$

$2.5 \quad$ A* Search . . . . . . . . . . . . . . . . . . . . 16

2.6 An Example for G-FRA* . . . . . . . . . . . . . . . . . . . . . 19

2.7 first-move table of a Node . . . . . . . . . . . . . . . . . 22

2.8 Basic and Two-Level Subgoals . . . . . . . . . . . . . . . . . . . 24

2.9 Construction of Simple Subgoal Graphs . . . . . . . . . . . . . 25

2.10 Construction of a Two-Level Subgoal Graph . . . . . . . . . . 30

2.11 Search with a Two-Level Subgoal . . . . . . . . . . . . . . . 31

3.1 Execution of MTSub - Incremental Search _ . . . . . . . . . 46

3.2 Early Termination Methods . . . . . . . . . . . . . . . . . 47

3.3 Execution of MTSub - Rebuilding a Search Tree . . . . . . . . . 48

$4.1 \quad$ Map Classes . . . . . . . . . . . . . . . . . . . . . 53

4.2 Experiments in $512 \times 512$ maps . . . . . . . . . . . . . . . . 59

4.3 Effects of Target Movement Strategy on MTSub . . . . . . . . . . 62

4.4 Run Times per Search for MTSub and R-Sub in Map AR0700SR . . 64

4.5 Run Time of MTSub and MTSCopa . . . . . . . . . . . . 66

4.6 Run Times per Search for MTSub and MTSCopa in Map AR0700SR 69

4.7 Moving Window Example . . . . . . . . . . . . . . . . 72 


\section{Chapter 1}

\section{Introduction}

A large number of applications, in video games, robotics, and virtual simulations, require agents to plan a complete path from their location to a target. For instance, a cop has to determine how to travel to a location when a robbery is taking place. Since the environment would most likely contain obstacles, the cop would need to account for these when planning his or her path. Following the shortest path would be ideal for the cop since it will take shorter time to catch the robber. This is the stationary path planning problem because the target location does not change. Solutions to this family of problem use Dijkstra's algorithm [1], which is an early optimal algorithm for the stationary path planning problem. Another optimal algorithm for the problem, A* [2], finds paths with fewer computations than Dijkstra's algorithm. This is because A* makes an informed search by using user provided heuristic values. These two algorithms are the backbone of the more recent algorithms such as Field $\mathrm{D}^{*}[3]$ which is used for the Mars Rover. 
In most applications, agents have to plan their path not only to a stationary target but also with respect to a moving target. In the previous example the robber may have fled the crime scene and the cop has to catch the robber. Even if the cop knows the location of the target at all times (with the help of a police helicopter for example) the cop needs to update his or her path to the robber according to the robbers known position. This type of problem is known as the Moving Target Search (MTS) problem. The MTS problem was first defined in 1991 [4], and has attracted a lot of attention $[5,6,7,8,9]$. In MTS problems, the agent and the target move at discrete time steps. The objective of the agent is to reach the target while minimizing the cost of the path taken. The information available to the agent (varying from full knowledge of properties such as the target location, obstacle locations), cost objective of an agent, and other requirements (e.g. response time, memory, preprocessing time) depend on partial or infrequent knowledge. Another example is an Unmanned Aerial Vehicle (UAV) operating in a dynamic (changing) environment that has imperfect information of the environment. However, it knows the location of the moving target (e.g. an aircraft carrier) at all times with the help of reports from a third party. The UAV searches for the safest path.

In this work, we focus on the moving target search problem where the agent has full knowledge of the search environment, which is static. This variant of MTS often arises in robotic applications that run in known environments and in video games. In this problem, when a change in a target's location is observed, agents need to examine their plan during execution. Namely, the original planned path has to be updated in order to obtain a better path. The speed with which these operations are executed is important because the agent must decide where to move next in real time. For 
example, in video games developed by Bioware Corp, the search time is restricted to $3 \mathrm{~ms}$ [10]. The quality of the updated solutions is also crucial because it directly affects the cost of reaching the target.

We introduce a new algorithm for this version of the problem - the Moving Target Search with Subgoal Graphs (MTSub). MTSub is the first algorithm for MTS that combines the advantages of incremental search and environment abstraction to decrease response times and preprocessing requirements without sacrificing optimality of the solutions. MTSub provides optimal solutions for MTS with low requirements for preprocessing and runtime.

\subsection{Contribution Summary}

We introduce an innovative, incremental search algorithm that uses environment abstraction to shorten response time of the agent without giving up cost minimal paths, Moving Target Search with Subgoal Graphs (MTSub).

Subgoal Graphs are an environment abstraction technique that select a subset of the original search environment by determining intersections that may contribute to cost minimal paths (subgoals). A cost minimal path can be found between any two subgoals with an A* search. Subgoals are similar to visibility graphs [11] in 2D planes. Searching in a Subgoal Graph requires fewer operations since it has fewer nodes and edges than the original graph. MTSub uses Subgoal Graphs to meet the real-time requirements. 
Since the paths between consequent discrete time steps are similar, MTSub attempts to update the path based on knowledge from the previous step. Incremental search plays a key role in MTSub and decreases its runtimes.

We proved that MTSub is an optimal algorithm for MTS. Our experiments that we conducted in four map domains with two target movement strategies showed that MTSub meets real-time requirements and it does not require heavy-weight preprocessing time or space. These results prove that MTSub is applicable to not only small environments but also to the larger ones.

We conducted experiments in four different map domains with two different target movement strategies. For each map, we used a hundred different initial locations for the agent and the target. Our experiments show that MTSub meets real time requirements of the applications and has low requirements for the preprocessing.

\subsection{Thesis organization}

Chapter 2 presents required concepts and background. Chapter 3 introduces MTSub, which is an innovative incremental algorithm for MTS with its variations. Chapter 4 presents the experiments and experiment methodology and results of analysis

demonstrating the effectiveness of MTSub in real life applications. Chapter 5 provides conclusions and future work. 


\section{Chapter 2}

\section{Background}

This chapter gives an overview of existing work and the Moving Target Search (MTS) problem. Several techniques and selected algorithms, which are directly related to this research work, are presented in more detail.

Moving Target Search (MTS) is a path planning problem where an agent attempts to reach a moving target [4]. This problem has direct applications in video games, dynamic robotics and virtual simulations. The problem can have different flavours depending in the problem parameters such as knowledge of the target location, weighted environment, and dynamic obstacles $[6,12,13]$.

For example, in some applications the environment is dynamic and the obstacles can change their location from time to time. Moreover, the agent may have partial knowledge of the environment [12]. The agent has to consider error in the sensors, changes in the environment and the target location to plan. We study an MTS problem where the environment does not have dynamic obstacles that change their 
location over time (static environment) and the location of the target is known at all the times. This version usually appears in the video games and in some of the robotics applications.

\subsection{Search Space Representation}

The environment in which the agent and the target operate consists of obstacles and free space. Different data structures can be used to represent the environment, which creates a search space. We explain two of the techniques that are mentioned in this work below.

\subsubsection{Grids}

A tessellation divides the space into equally sized cells, such as triangles, rectangles and hexagons. For the sake of simplicity, square cells are widely preferred in path planning $[4,7,14,15]$. Phases of a grid representation, namely initial space, partitioning and, final grid is depicted in Figure 2.1. In this thesis, the suggested algorithm and competing algorithms use 8-connected grids, which are demonstrated in Figure 2.2 .

In 8-connected grids, the center of each tile in the grid is declared as a node. Each node is connected to its four cardinal and four diagonal neighbours with an undirected edge. Cardinal moves are allowed between adjacent nodes if both corresponding nodes are unblocked. Diagonal moves are allowed if both nodes are unblocked and the two corresponding cardinal nodes are also unblocked (here we assume the agent is as large 


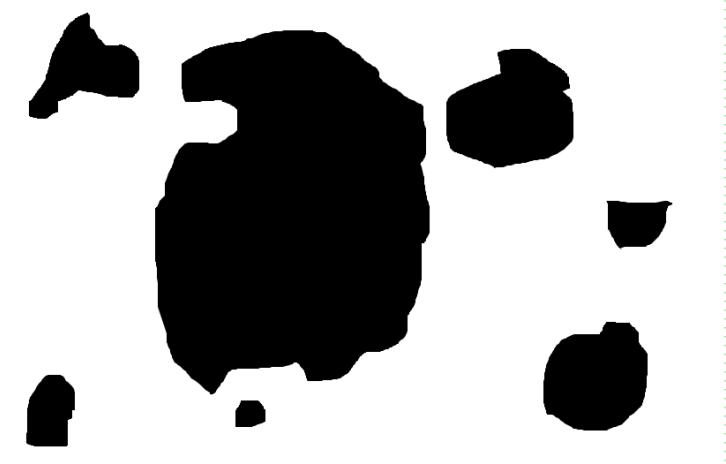

(a) Search Space

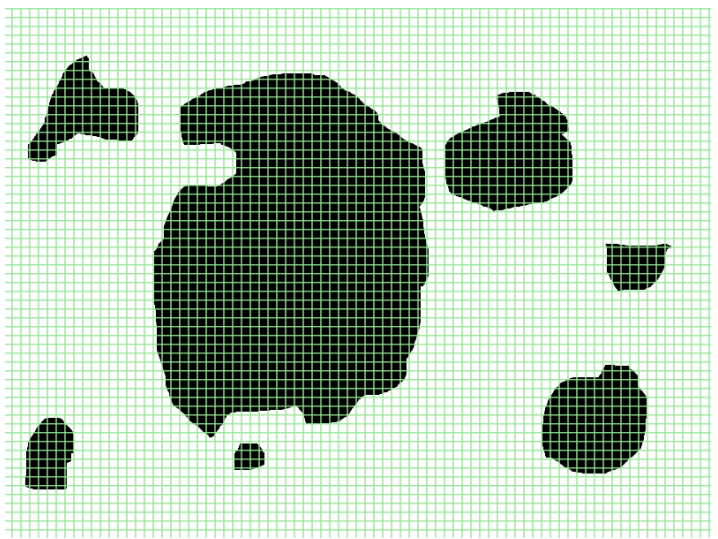

(b) Partitioned Search Space

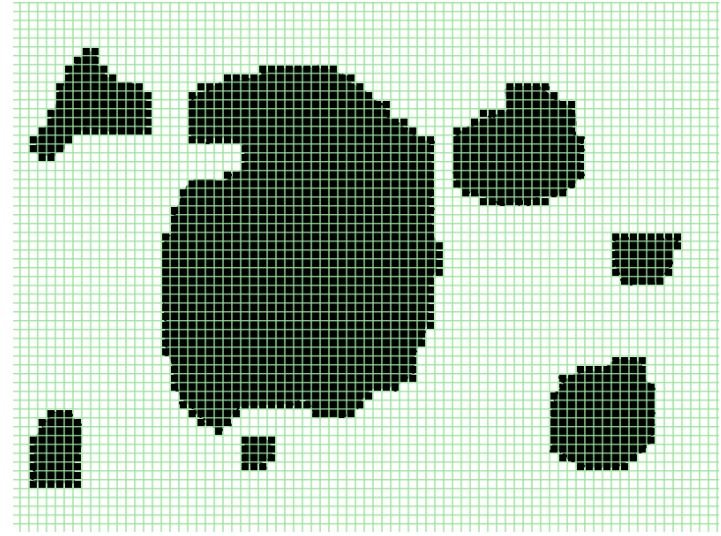

(c) Grid

Figure 2.1: Grid Representation

as a grid cell). The costs of cardinal and diagonal moves are 1 and $\sqrt{2}$, respectively.

\subsubsection{Navigation Meshes}

Navigation Meshes (NavMesh) are popular in video games for path planning operations [17]. They divide a search space into non-overlapping polygons. A graph is obtained by declaring a point in each cell as a node (e.g. the center of the cell) and connecting them with undirected edges. Edge costs are determined by Euclidean 


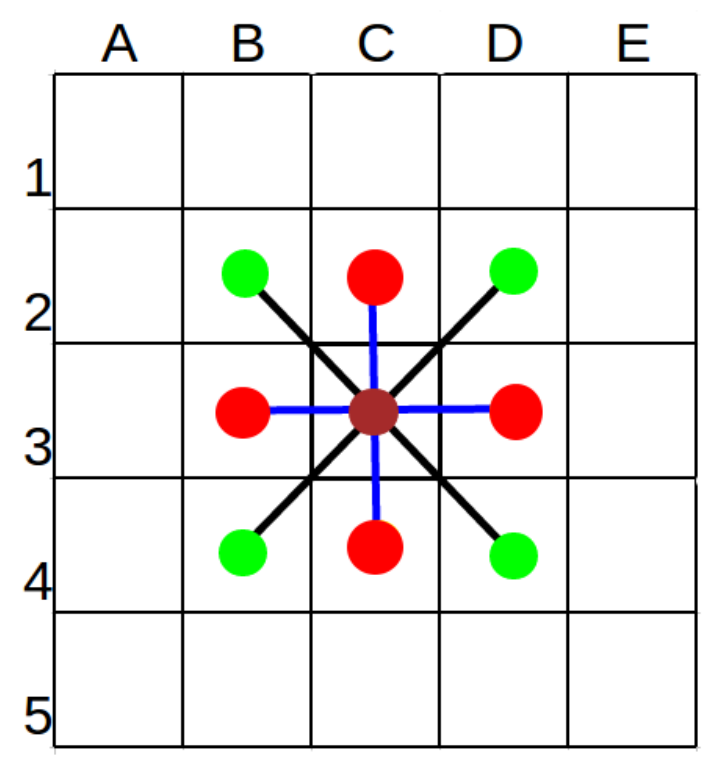

Figure 2.2: 8 Connected Grid: Dark red dot represents a node in the centre of the grid cell C3. Green dots and red dots are used to show diagonal and cardinal neighbours of C3 respectively. Similarly, blue lines show cardinal edges with the cost of 1 where black lines show diagonal edges with the cost of $\sqrt{2}$.

distance between corresponding nodes. Creation of such a graph directly affects the quality of cost minimal paths. Some methods and resulting paths are depicted in Figure 2.3. NavMesh lets developers represent a search environment with smaller graphs than would be possible with grids when the environment has large free spaces. However, quality of the paths may suffer. This thesis mentions NavMesh when discussing possible extensions of the suggested algorithm. 


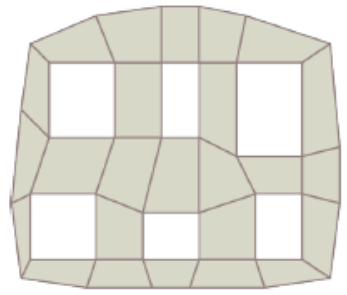

(a) Polygons

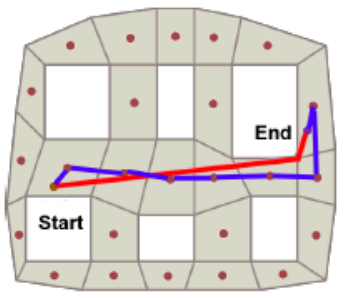

(b) Center Points

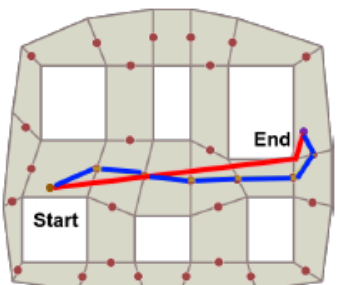

(c) Edge Points

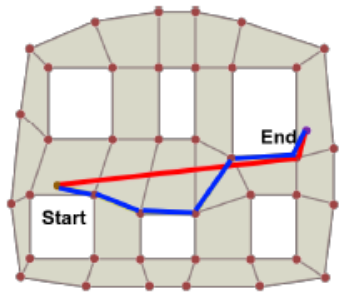

(d) End Points

Figure 2.3: Navigation Mesh Figures [16]: Four figures are given to depict different strategies for selecting nodes. Figure 2.3a shows non-overlapping polygons. Figures $2.3 \mathrm{~b}, 2.3 \mathrm{c}$ and $2.3 \mathrm{~d}$ show node selections. Each blue line depicts a path over a Navigation Mesh while each red line depicts a cost-minimal path.

\section{$2.2 \quad$ Problem Definition}

The problem is defined as follows: Given a graph $G=(V, E)$, representing an 8connected grid environment, an agent $A$ and a target $T$, positioned at $s_{A}$ and $s_{T}$ respectively, find an optimal route from $A$ to $T$ :

- $A$ and $T$ can move at discrete time steps.

- At each time step $t_{i}, T$ positioned at $u=\operatorname{position}\left(T, t_{i}\right)$ can either stay put or move to node $v$ where $v \in$ neighbour $(u)$.

- At each time step $t_{i}, A$, positioned at $\left(p=\operatorname{position}\left(A, t_{i}\right)\right)$, can detect the position of $T\left(u=\operatorname{position}\left(T, t_{i}\right)\right)$, compute a path $\Pi(p, u)$ and move to a node $w$ where $w \in$ neighbour $(u) \wedge w \in \Pi(p, u)$.

\subsubsection{Optimal Solution}

An optimal route is a route from $\operatorname{position}\left(A, t_{o}\right)$ to $\operatorname{position}\left(T, t_{k}\right)$, where $t_{o}$ is the time that $A$ and $T$ begin moving, $t_{k}$ is the time at which $A$ reached $T, \forall t_{i}$, and $t_{o} \leq t_{i} \leq t_{k}$, 


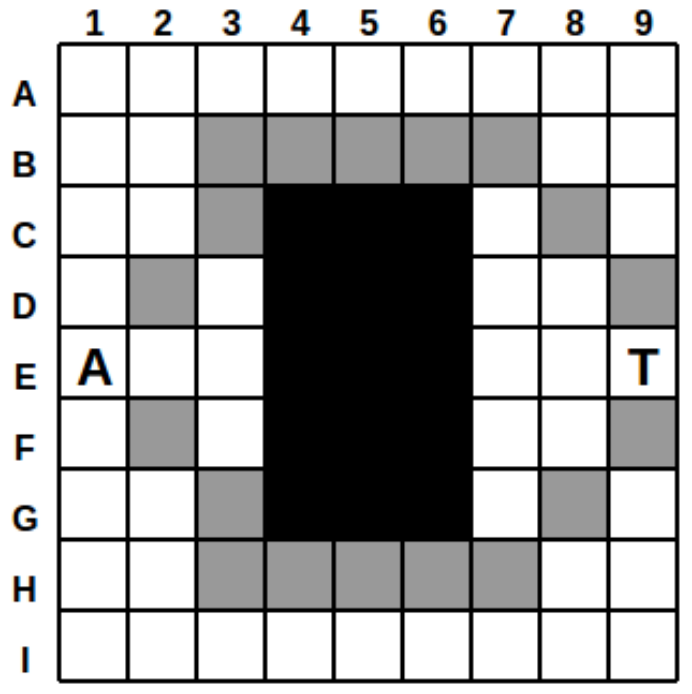

(a) Positions at time $t_{i}$

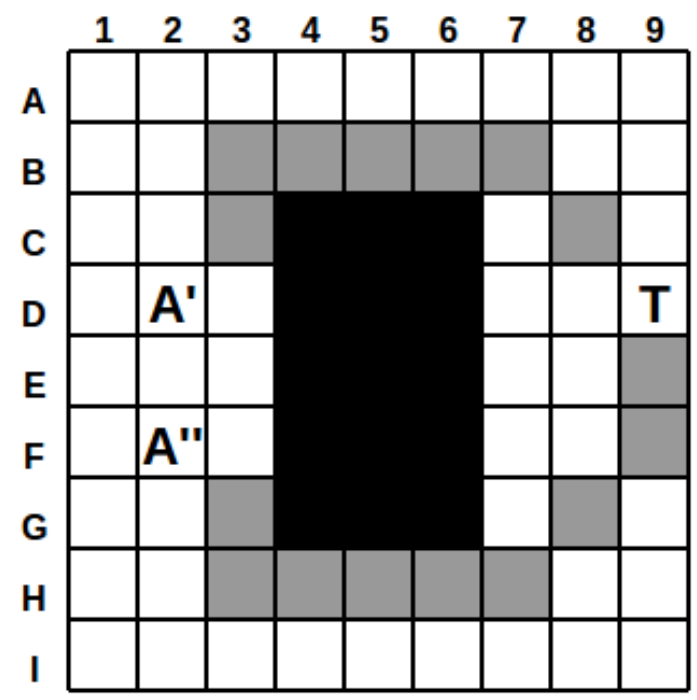

(b) Positions at time $t_{i+1}$

Figure 2.4: a. Two possible paths with the same cost that the agent, $A$, can take to reach the target $T$ at time $t_{i}$; b. Positions of $A$ and $T$ at time $t_{i+1}$ after $A$ chose one of the two paths arbitrarily.

position $\left(A, t_{i}\right) \in \Pi\left(\right.$ position $\left(A, t_{i-1}\right)$,position $\left.\left(T, t_{i-1}\right)\right)$ where $\Pi$ is a cost minimal path.

Note that following an optimal route may not minimize the cost of reaching the target. Figure 2.4 depicts a search environment at time $t_{i}$ and $t_{i+1}$ where the black cells represent obstacles, 'A' and ' $\mathrm{T}$ ' show $s_{A}$ and $s_{T}$, respectively. Figure 2.4a provides an example of two obstacle free paths that have the same cost. Figure 2.4b shows that the agent's random choice of paths at time $t_{i}$ may not be the best choice to get closer to the target. This implies that following a cost minimal path at any time $t$ may not minimize overall cost of reaching a target since the strategy does not have any information about future actions of the target. However, experiments show that following an optimal route decreases the cost of reaching the target in most cases $[9,13]$. This example also implies that following a cost minimal path does not 
guarantee to catch a target if an agent and a target have the same speed. This is because the target may circle around an obstacle and this may make it impossible for the agent to get closer to the target.

\subsection{Previous Work}

This section gives an overview of existing work regarding Moving Target Search. The selected algorithms are then discussed further to provide a better comprehension of the contributions of this thesis.

Moving Target Search (MTS) is a path planning problem where an agent attempts to reach a moving target [4].

There are generally two kinds of strategies for solving MTS problems with search algorithms [12]:

- Offline techniques $[6,18,19,20]$ consider all possible locations of the agent and the target in the environment to determine the best plan prior to movement of the agent. These techniques are not applicable to large environments due to large numbers of possible future actions of a target.

- Online techniques find a solution according to current information and update the existing plan when changes occur. They solve a series of path planning problems during plan execution to react to the target movement. Earlier online algorithms $[4,5]$ find a prefix of the path with a bounded search. Therefore, they can work under strict time bounds. However, the cost of reaching the target location is high since they make short-sighted decisions. As such, it 
is hard to determine the existence of a path between an agent and a target. Subsequent online algorithms $[7,12,13]$ find a complete path from the agent towards the target and update the path during the search. Fringe Retrieving $\mathrm{A}^{*}\left(\mathrm{FRA}^{*}\right)$ [7] and Generalized Fringe Retrieving A* (G-FRA*) [12] are two algorithms that calculate cost minimal, complete paths between the current positions of the agent and the target. The overall cost of reaching the target using G-FRA* or FRA* is smaller than that of earlier online algorithms that find only a prefix of the path. Nevertheless, they cannot run under strict time bounds. Incremental Any time Repairing A* (I-ARA*) [13] can compute paths between current locations of the agent and the target under user defined time limits. However, the paths may not be cost minimal. Thus, the cost of reaching the target is higher [9] than algorithms that find cost minimal paths between current positions of the agent and the target. A more recent paper introduced Moving Target Search with Compressed Databases (MtsCopa) [9], which exploits preprocessing to speed up online search without sacrificing cost minimal paths between current locations of the agent and the target. Unfortunately,

preprocessing time and space requirements of the algorithm are substantially high (e.g., over eleven hours of preprocessing time and 23MB of space are required for a 320 x 320 video game map). The performance of MtsCopa against the latest algorithms for fast first move queries is discussed in [21].

\subsection{1 $\mathrm{A}^{*}$}

$A^{*}[2]$ is a search algorithm that uses heuristics for calculating cost minimal paths between stationary locations. $A^{*}$ is a heuristic version of the well-known Dijkstra's 
algorithm. User provided heuristics enables the algorithms to find cost minimal paths while checking fewer nodes. Therefore, the cost minimal searches are faster with heuristics. All of the dynamic algorithms for MTS, including our algorithm, utilizes heuristic search. We would like to discuss $\mathrm{A}^{*}$ to provide background to heuristic search.

\section{Description}

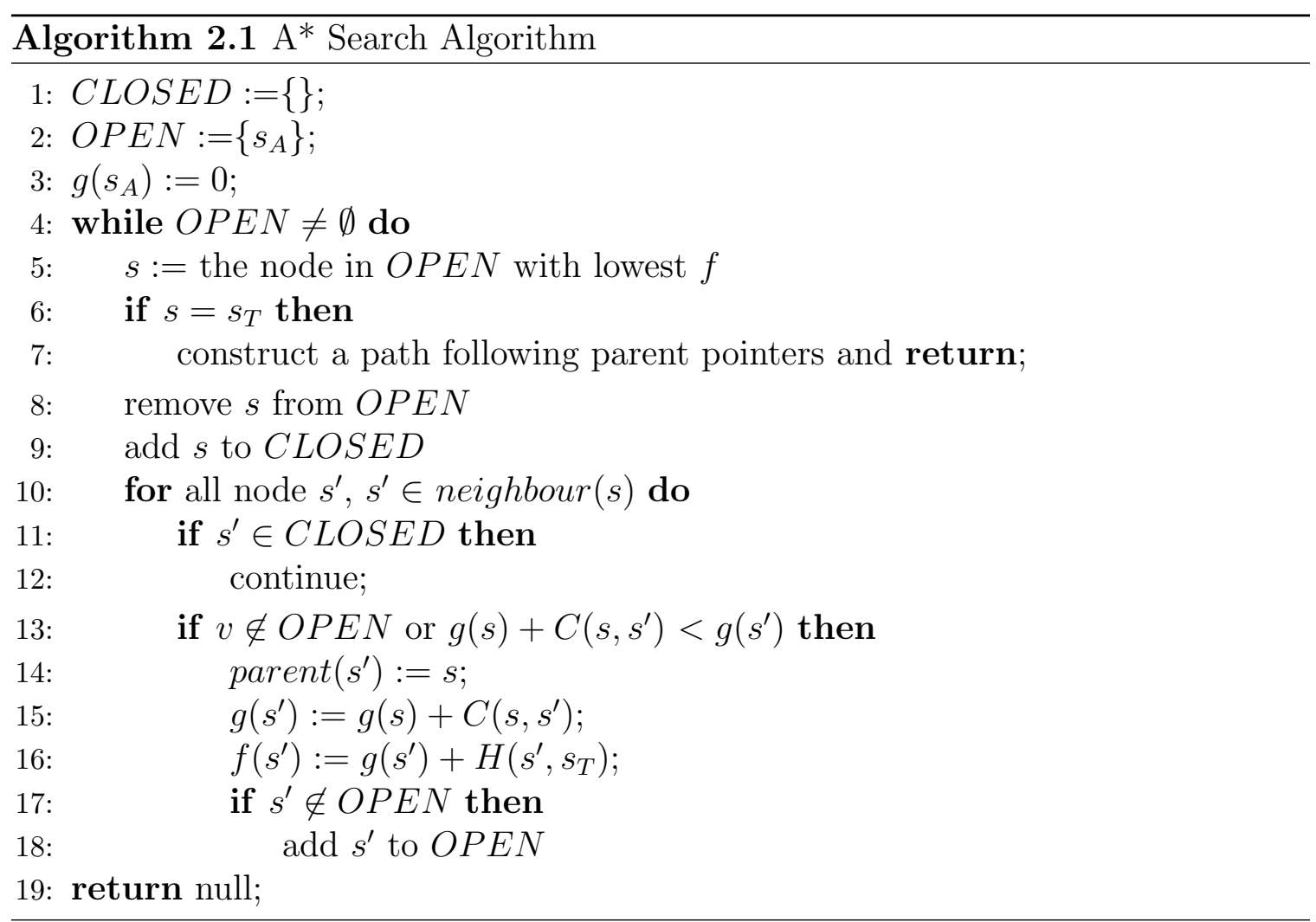

This section explains $A^{*}$ search algorithm. The following description of the $A^{*}$ algorithm will follow the pseudo-code provided in Algorithm 2.1.

$\mathrm{A}^{*}$ keeps track of four values for each node $s \in S$ : 
1. Approximation cost - $h(s)$ denotes user provided approximation of $C\left(s, s_{T}\right)$ where $s, s_{T} \in S$. The value $h(s)$ is computed by the function $H\left(s, s_{T}\right) . \mathrm{A}^{*}$ can return cost minimal paths only if admissible heuristics are provided. Every function $H$ that has the triangle inequality property $\left(H\left(s, s^{\prime \prime}\right) \leq H\left(s, s^{\prime}\right)+\right.$ $H\left(s^{\prime}, s^{\prime \prime}\right)$ for all $\left.s, s^{\prime}, s^{\prime \prime} \in S\right)$ is admissible [22]. Function $H\left(s, s^{\prime}\right)$ calculates octile distances between $s$ and $s^{\prime}$. An octile distance is the shortest distance between $s$ and $s^{\prime}$ in an 8 connected grid as if there were no obstacles.

2. Calculated value for $C\left(s_{A}, s\right)$ - this value is denoted $g(s)$. Initially, t is assigned a zero for $g\left(s_{A}\right)$ (line 3 ) and infinity for each node $s \in S$ other than $s_{A}$ before the search.

3. Hierarchical cost of reaching the target - this value is denoted by $f(s)$ where $f(s)=g(s)+h(s)$.

4. Parent of $s$ - the value, parent $(s)$ denotes the node that is the parent of $s$ in the search tree. Initially, parent $(u)=$ NULL.

$\mathrm{A}^{*}$ also uses two lists:

1. $O P E N$ list - this list maintains the nodes that are going to be evaluated. Initially, it has only $s_{A}$ as a member (line 2).

2. CLOSED list - this list contains all the nodes that were evaluated (expanded).

At each iteration of $\mathrm{A}^{*}$, a node $s$ with minimum $f$ value is extracted from $O P E N$ and added to CLOSED (line 4). Each node $s^{\prime} \in \operatorname{neighbour}(s)$ is evaluated as follows: if $g(s)+C\left(s, s^{\prime}\right)<g\left(s^{\prime}\right)$ then $g\left(s^{\prime}\right)=g(s)+C\left(s, s^{\prime}\right)$ and parent $\left(s^{\prime}\right):=s($ lines $13-16)$. 
Node $s^{\prime}$ is then added to the $O P E N$ list if it is not already in $O P E N$ (line 18). A* terminates and returns a cost minimal path if $s_{T}$ is expanded (line 7). If the OPEN list is empty and $s_{T}$ is not expanded, A* terminates and reports that there is not an obstacle free path between $s_{A}$ and $s_{T}$ (line 19).

\begin{tabular}{c|c|c|c|c|c|c|c|c|c|c|} 
& $\mathrm{A} 1$ & $\mathrm{~A} 2$ & $\mathrm{~A} 3$ & $\mathrm{~A} 4$ & $\mathrm{~B} 1$ & $\mathrm{~B} 3$ & $\mathrm{~B} 4$ & $\mathrm{C} 1$ & $\mathrm{C} 2$ & $\mathrm{C} 4$ \\
\hline$h$ values & 3.5 & 2.5 & 1.5 & 1 & 3 & 1 & 0 & 3.5 & 2.5 & 1
\end{tabular}

Table 2.1: $h$ values for the unblocked cells

\begin{tabular}{|c|c|c|c|c|c|c|c|c|c|c|c|c|c|c|c|c|c|c|c|c|}
\hline & \multicolumn{2}{|c|}{ A1 } & \multicolumn{2}{|c|}{$\mathrm{A} 2$} & \multicolumn{2}{|c|}{ A3 } & \multicolumn{2}{|c|}{$\mathrm{A} 4$} & \multicolumn{2}{|c|}{$\mathrm{B} 1$} & \multicolumn{2}{|c|}{ B3 } & \multicolumn{2}{|c|}{$\mathrm{B} 4(\mathrm{~T})$} & \multicolumn{2}{|c|}{$\mathrm{C} 1(\mathrm{~S})$} & \multicolumn{2}{|c|}{$\mathrm{C} 2$} & \multicolumn{2}{|c|}{$\mathrm{C} 4$} \\
\hline & $g$ & $f$ & $g$ & $f$ & $g$ & $f$ & $g$ & $f$ & $g$ & $f$ & $g$ & $f$ & $g$ & $f$ & $g$ & $f$ & $g$ & $f$ & $g$ & $f$ \\
\hline Iteration 1 & $\infty$ & $\infty$ & $\infty$ & $\infty$ & $\infty$ & $\infty$ & $\infty$ & $\infty$ & $\infty$ & $\infty$ & $\infty$ & $\infty$ & $\infty$ & $\infty$ & 0 & 3.5 & $\infty$ & $\infty$ & $\infty$ & $\infty$ \\
\hline Iteration 2 & $\infty$ & $\infty$ & $\infty$ & $\infty$ & $\infty$ & $\infty$ & $\infty$ & $\infty$ & 1 & 4 & $\infty$ & $\infty$ & $\infty$ & $\infty$ & 0 & 3.5 & 1 & 3.5 & $\infty$ & $\infty$ \\
\hline Iteration 3 & $\infty$ & $\infty$ & $\infty$ & $\infty$ & $\infty$ & $\infty$ & $\infty$ & $\infty$ & 1 & 4 & $\infty$ & $\infty$ & $\infty$ & $\infty$ & 0 & 3.5 & 1 & 3.5 & $\infty$ & $\infty$ \\
\hline Iteration 4 & 2 & 5.5 & $\infty$ & $\infty$ & $\infty$ & $\infty$ & $\infty$ & $\infty$ & 1 & 4 & $\infty$ & $\infty$ & $\infty$ & $\infty$ & 0 & 3.5 & 1 & 3.5 & $\infty$ & $\infty$ \\
\hline Iteration 5 & 2 & 5.5 & 3 & 5.5 & $\infty$ & $\infty$ & $\infty$ & $\infty$ & 1 & 4 & $\infty$ & $\infty$ & $\infty$ & $\infty$ & 0 & 3.5 & 1 & 3.5 & $\infty$ & $\infty$ \\
\hline Iteration 6 & 2 & 5.5 & 3 & 5.5 & 4 & 5.5 & $\infty$ & $\infty$ & 1 & 4 & $\infty$ & $\infty$ & $\infty$ & $\infty$ & 0 & 3.5 & 1 & 3.5 & $\infty$ & $\infty$ \\
\hline Iteration 7 & 2 & 5.5 & 3 & 5.5 & 4 & 5.5 & 5 & 6 & 1 & 4 & 5 & 6 & 5.5 & 5.5 & 0 & 3.5 & 1 & 3.5 & $\infty$ & $\infty$ \\
\hline Iteration 8 & 2 & 5.5 & 3 & 5.5 & 4 & 5.5 & 5 & 6 & 1 & 4 & 5 & 6 & 5.5 & 5.5 & 0 & 3.5 & 1 & 3.5 & $\infty$ & $x$ \\
\hline
\end{tabular}

Table 2.2: $g$ and $f$ values for the unblocked cells

Figure 2.5 shows a simple example of an $\mathrm{A}^{*}$ search. The environment is represented with an 8 connected grid where an agent is allowed to move diagonally only if corresponding cardinal tiles are free. Moving cost for cardinal moves is 1 and for diagonal moves it is 1.5 for the sake of simplicity. Figure 2.5a shows the search graph. Black cells are occupied. An agent and a target are positioned in C1 and B4, respectively (indicated with an 'S' and 'T'). Grid nodes are green if they are in $O P E N$ or red if they are in CLOSED. Table 2.2 shows $g$ and $f$ values of the grid nodes for each iteration. Table 2.1 shows $h$ values for the grid nodes.

A* executes as follows:

1. In the beginning of $\mathrm{A}^{*}$ (Figure $2.5 \mathrm{~b}$ ), $g\left(s_{A}\right)$ is assigned a zero and $s_{A}$ is inserted 


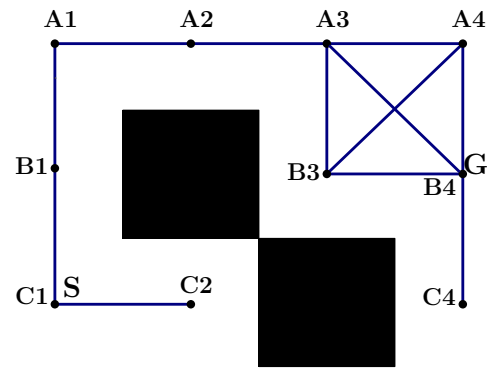

(a) Search Graph

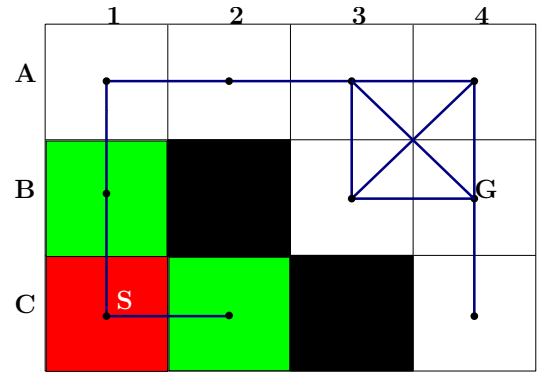

(c) Iteration 2

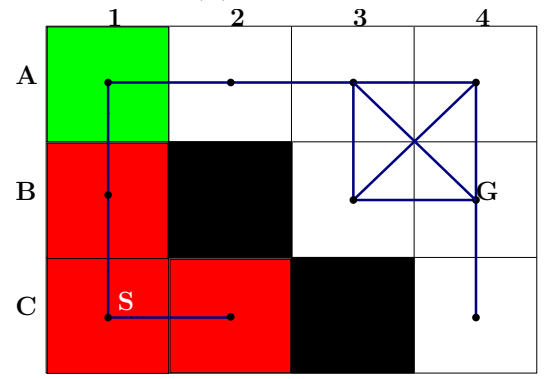

(e) Iteration 4

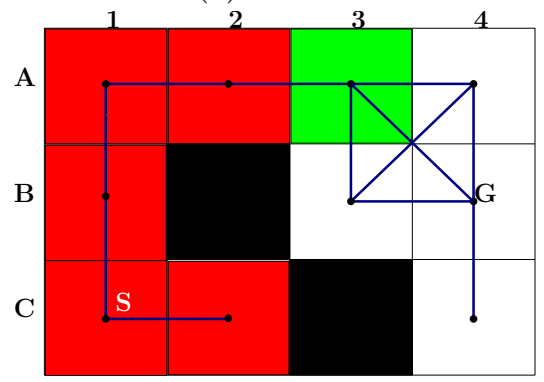

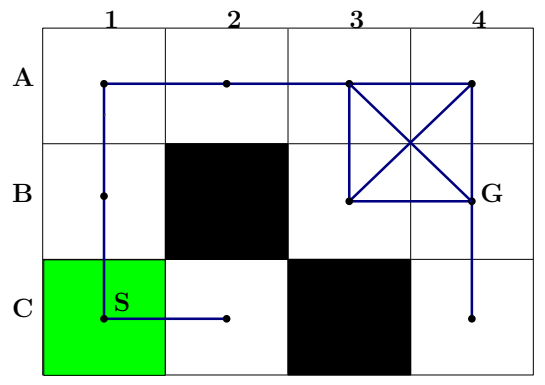

(b) Iteration 1

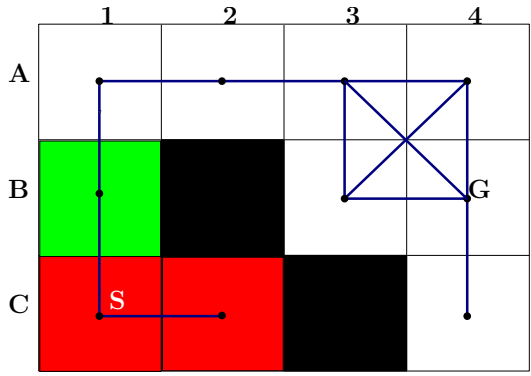

(d) Iteration 3

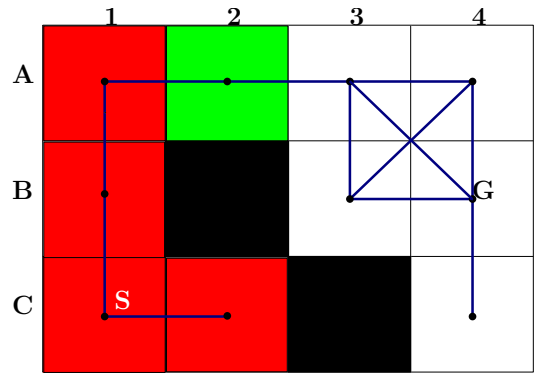

(f) Iteration 5

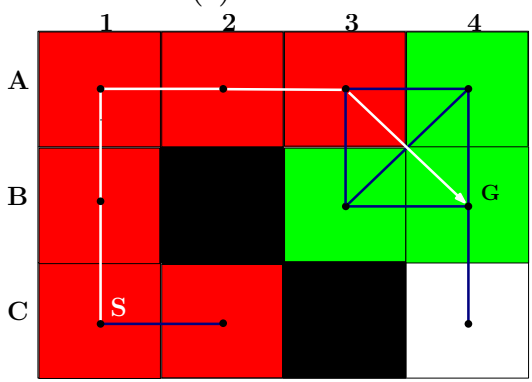

(h) Iteration 7-8

(g) Iteration 6

Figure 2.5: A* Search 
into $O P E N$ whereas $C L O S E D$ is initially empty.

2. In the second iteration (Figure 2.5c), $s_{A}$ is expanded since it is the only node in $O P E N$. Neighbours of $s_{A}$, namely $\mathrm{B} 1$ and $\mathrm{C} 2$, are inserted into OPEN after their $g$ values are set to 1 .

3. In the third iteration (Figure 2.5d), C2 is expanded because it is the node with smallest $f$ value in $O P E N(f(C 2)=3.5, f(B 1)=4)$. It has only one neighbour, which is $s_{A}$. Since C2 doesn't offer a smaller $g$ value, A* doesn't update $s_{A}$.

4. In the fourth iteration (Figure 2.5e), the only node in $O P E N, \mathrm{~B} 1$ is expanded. $\mathrm{A} 1$, the only neighbour of $\mathrm{B} 1$, is inserted into $O P E N$ after $g(A 1)$ is updated. Please note that A2 is not a neighbour of B1 because B2 is occupied.

5. In the fifth iteration (Figure 2.5f), the only node in $O P E N$, A1 is expanded. $\mathrm{A} 2$, only neighbour of $\mathrm{A} 1$, is inserted into $O P E N$ after $g(A 2)$ is updated.

6. In the sixth iteration (Figure $2.5 \mathrm{~g}$ ), the only node in $O P E N, \mathrm{~A} 2$ is expanded. A3, only neighbour of A2, is inserted into $O P E N$ after $g(A 3)$ is updated.

7. In the seventh iteration (Figure $2.5 \mathrm{~h}$ ), the only node in $O P E N, \mathrm{~A} 3$ is expanded. A4, B3 and $s_{T}$ are added to OPEN after their $g$ values are updated. Please note that, $\mathrm{A}^{*}$ doesn't terminate until $s_{T}$ is expanded.

8. In the final iteration (Figure $2.5 \mathrm{~h}$ ), $s_{T}$ is expanded since it is the node with the lowest $f$ value in $O P E N\left(f(A 4)=6, f(B 3)=6, f\left(s_{T}\right)=5.5\right)$. A cost minimal path is returned by following parent pointers (indicated with a white arrow). 


\section{Using $A^{*}$ for MTS: Repeated A*}

Algorithms for planning a path between static locations can be used to solve MTS problems. This is because at any time $t$, MTS requires a path between locations of $s_{A}$ and $s_{T}$.

$\mathrm{A}^{*}$ can be used to update a path of an agent if necessary (Algorithm 2.2: line 4). Repeating $A^{*}\left(R-A^{*}\right)$ is used to denote an $A^{*}$ search that is used for MTS. R-A* doesn't need preprocessing time or extra space for recording past information regarding a target or an environment. Therefore, it doesn't exploit any similarities between subsequent problems. However, it can operate in highly dynamic environments without performance degradation.

\subsubsection{Generalized Fringe Retrieving $A^{*}$}

G-FRA* [23] is an incremental search algorithm for MTS in static environments. It has the fastest average response time among optimal algorithms that do not exploit preprocessing [15]. G-FRA* constructs a search tree with an $A^{*}$ search. Then, any time an agent needs to update its plan, G-FRA* modifies the existing search tree. G-FRA* continues to expand the search tree until it finds a cost minimal path.

\section{Description}

G-FRA* has six steps which are explained as follows:

I Starting A* Directly: This step is executed if $s_{A}$ is not changed $\left(s_{A}=\right.$ 


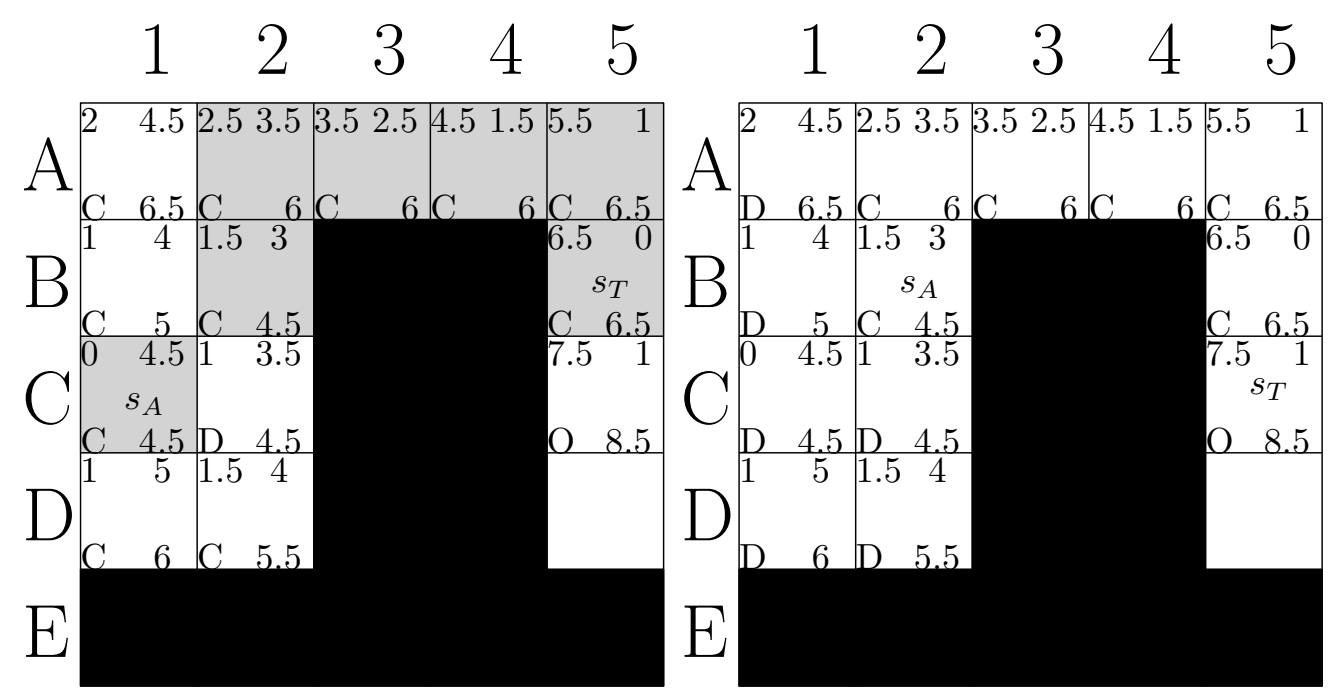

(a) Initial A* Search

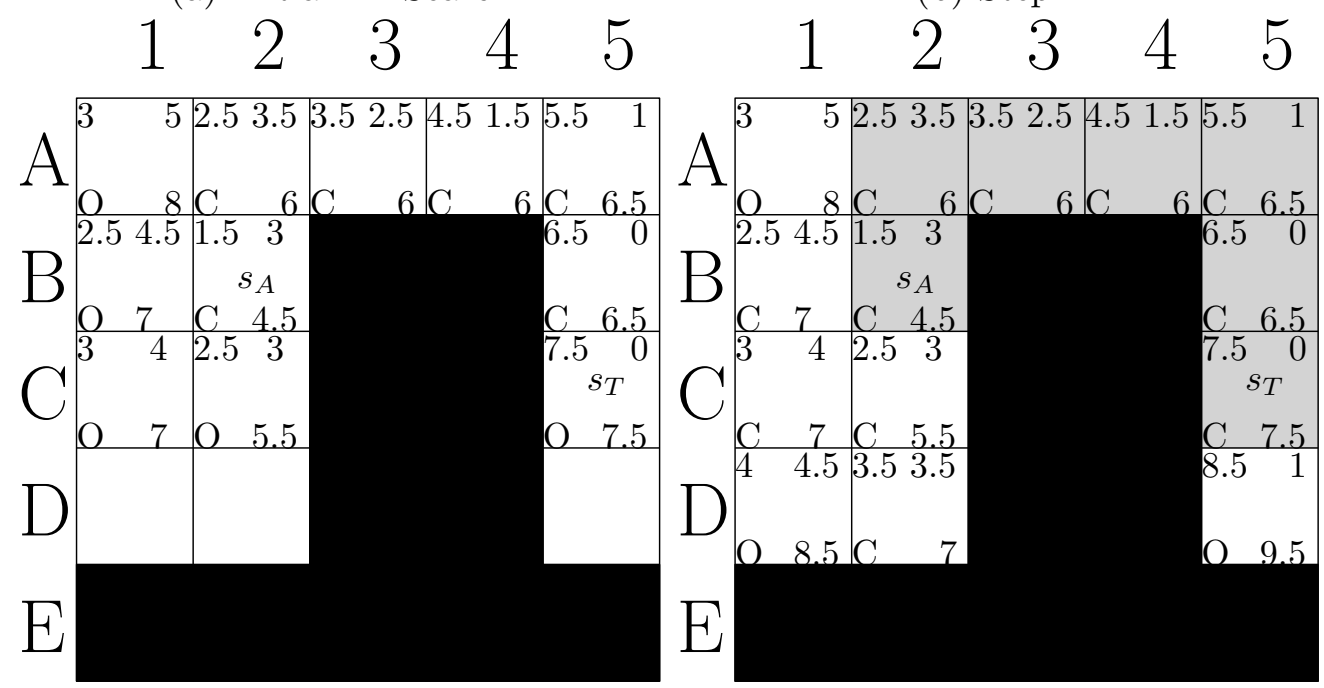

(c) Step 4

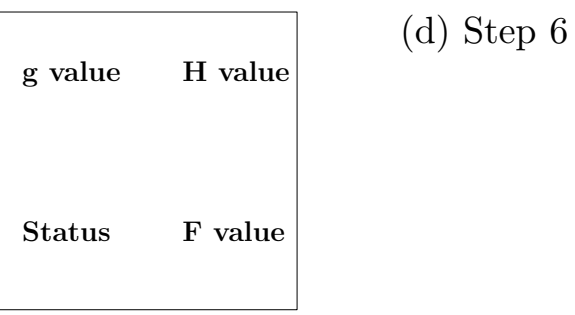

(e) Legend

Figure 2.6: An Example for G-FRA*: Figures show 8-connected grids where cardinal and diagonal costs are 1 and 1.5 for simplicity. Black cells are occupied and a legend for free cells is given in Figure 2.6e. $s_{A}$ and $s_{T}$ shows current positions of the agent and the target, respectively. Found paths are depicted with gray cells. 
position $(A, t-1)$ ). If $s_{T} \in C L O S E D$, a cost minimal path can be found following parent pointers of the nodes in CLOSED. However, if $s_{T} \notin C L O S E D, h$ values of the nodes in $O P E N$ must be updated accordingly to $s_{T}$. This operation is done in step 5. Following the execution of step 5, G-FRA* finds a path with an $A^{*}$ search.

II Deleting Nodes: In the second step, G-FRA* removes the nodes $s$ from $C L O S E D$ and $O P E N$, if $s$ is not in the search tree rooted at $s_{A}$. In other words, it is not possible to find a cost minimal path between $s_{A}$ and $s$ by following parent pointers starting from $s$. All of the removed nodes are added to a new list, which is called DELETED.

III Terminate Early: If the $s_{T}$ is already in CLOSED, G-FRA* finds a cost minimal path trivially by following parent pointers of the nodes. It is important to note that even if a cost minimal path $\Pi\left(s_{A}, s_{T}\right)$ can be found, OPEN may be incomplete and it must be completed in the following step.

IV Complete OPEN: G-FRA* adds new nodes to OPEN since it is not complete after actions taken in step 2. A node $s \in D E L E T E D$ is added to the OPEN list if $s \in$ neighbour $\left(s^{\prime}\right)$ where $s^{\prime} \in C L O S E D$. The values, $g(s)$ and parent $(s)$ are changed accordingly. DELETED list is empty afterwards. After this step, the $O P E N$ list is complete since it is guaranteed that every node on the outer perimeter of the CLOSED list is in the OPEN list.

V Update Heuristic Values: This step is executed only if $s_{T} \notin C L O S E D$ and $s_{A}=\operatorname{position}(A, t-1)$. G-FRA* updates $h$ values of the nodes in OPEN with respect to $s_{T}$. Therefore, $f$ values are then updated accordingly. 
VI Starting $\mathbf{A}^{*}$ : In the final step, G-FRA* initiates an $\mathrm{A}^{*}$ search with complete $O P E N$ and $C L O S E D$ to return a cost minimal path.

Figure 2.6 shows how G-FRA* works. An initial search is done starting with an $\mathrm{A}^{*}$ (Figure 2.6a). Since the agent has moved, nodes that are not rooted at $s_{A}$ are removed from CLOSED and OPEN and added to DELETED (Figure 2.6b). G-FRA* continues with step 3 because $s_{T}$ is not in CLOSED. In step 4, nodes in CLOSED are iterated through and relevant nodes are added to OPEN with updated $g$ and $f$ values (Figure 2.6c). Finally, G-FRA* finds a cost minimal path with completed OPEN and CLOSED (Figure 2.6d).

\subsubsection{MTS with Compressed Path Databases}

MTSCopa [9] is an algorithm for MTS that exploits preprocessing. MTSCopa builds Compressed Path Databases(CPD's) during a preprocessing step to answer path queries of an agent at runtime. MTSCopa has the smaller average and maximum response times than any other algorithm for MTS [9] and its ability to return the next move for an agent makes MTSCopa particularly interesting for MTS. Unfortunately, it is not possible to apply state-of-the-art MTSCopa to large environments due to its significant space and preprocessing time requirements.

\section{Description}

During the preprocessing step, MTSCopa creates a CPD as follows: Given a graph

$G=(V, E)$ and $S$ (a list of unblocked nodes in $V$ ), MTSCopa calculates one-to-all cost minimal paths with Dijkstra's algorithm for all $s$ where $s \in S$. Only first moves 


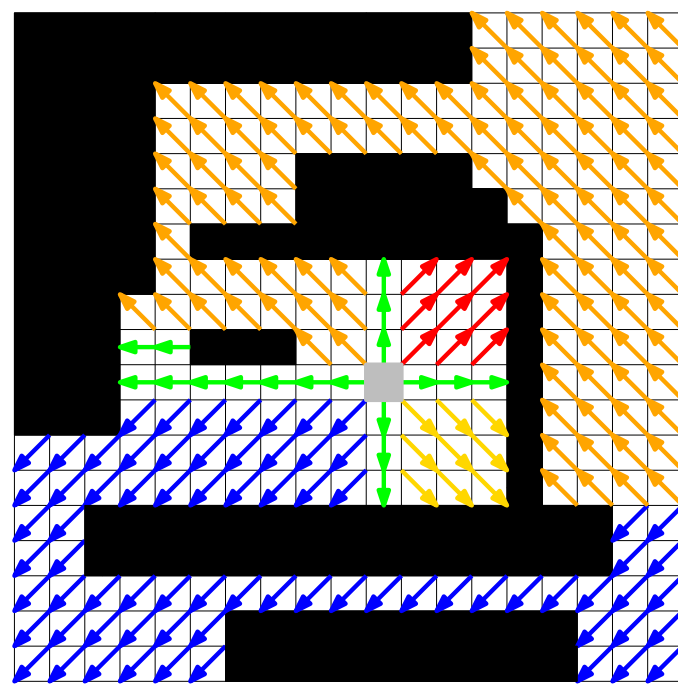

(a) First Move Data

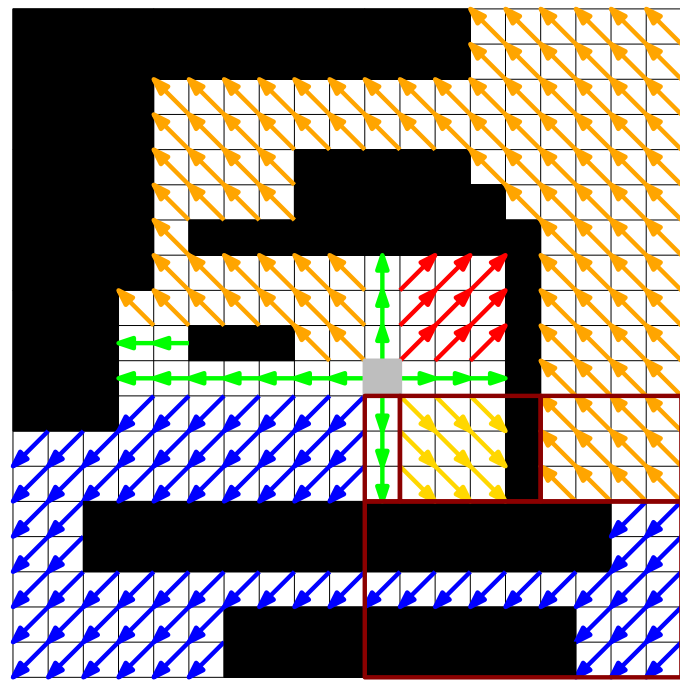

(b) Selection of Rectangular Regions

Figure 2.7: first-move table of a Node: First possible moves of the grey cell are depicted with arrows of different colors. In Figure 2.7b, four rectangular regions for south east sector are depicted with red lines.

of cost minimal paths are stored in what author's call, first-move table. Figure 2.7a shows an example of the data that is stored for a node $u$ in a first-move table. For all $s$, the first move data that is stored in a first-move table is compressed to decrease space requirements. To do so, the map is divided into, at most, four sectors (North-East, North-West, South-East, South-West) depending on the location of $s$. The algorithm identifies rectangular regions that contain the same first moves in each sector with a recursive approach [24] (Figure 2.7b). Time complexity of the preprocessing step of MTSCopa is $O\left(N^{2} \log (N)\right)$ where $N$ is number of unblocked grid cells. It is important to note that operations during the preprocessing step do not need to be done in a sequential fashion. Therefore, it is possible to gain time with parallel computing techniques.

Retrieving a next move from a CPD is straight-forward and requires no run-time 
search for a path. Depending on the location of a target, the sector of the next move is determined from the first-move table. Once done, the algorithm searches in rectangular regions to find where the target location belongs. This search (a rectangular check) is a constant time operation and finding desired rectangle takes at most $O(C N)$ where $C$ is constant time for a check operation and $N$ is the number of rectangular areas in a sector. Amortized time is much better if rectangular areas are sorted according to their size.

\subsubsection{A Generic Framework for MTS}

A generic framework for MTS is first introduced in [25]. We made small changes and present a pseudo-code for a generic algorithm to emphasize common resemblances between algorithms for MTS. The algorithm can be seen in Algorithm 2.2. In this algorithm, an agent is always aware of a target and the environment is static. Since the environment is known to the agent, it can be processed to gain advantage (line 1). The agent continues the search until it catches the target (line 2). The agent has to observe the environment at every time $t$ to obtain required data (line 3 ). That data is used to update the current path to react to the target's movements (line 4). Then, the agent simply follows the path (line 5).

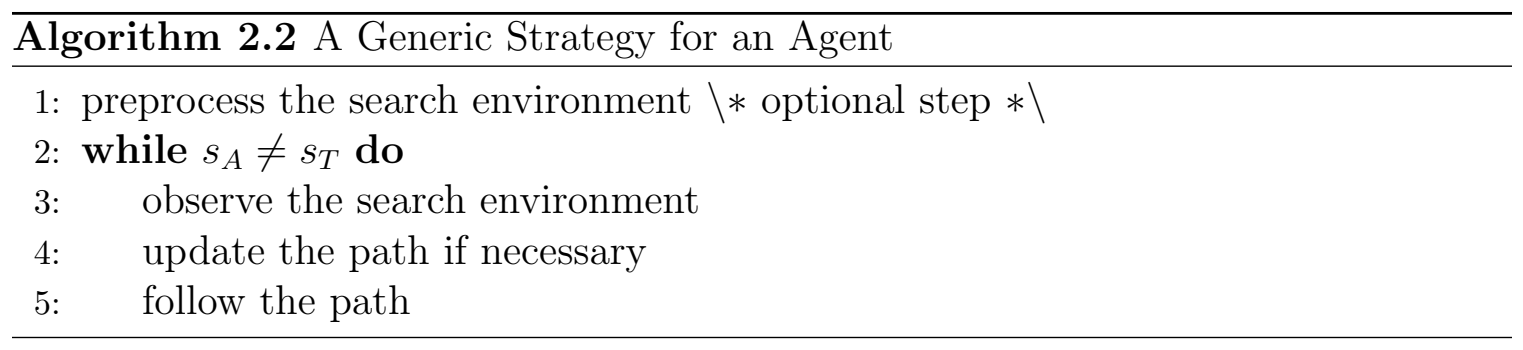




\subsubsection{Subgoal Graphs}

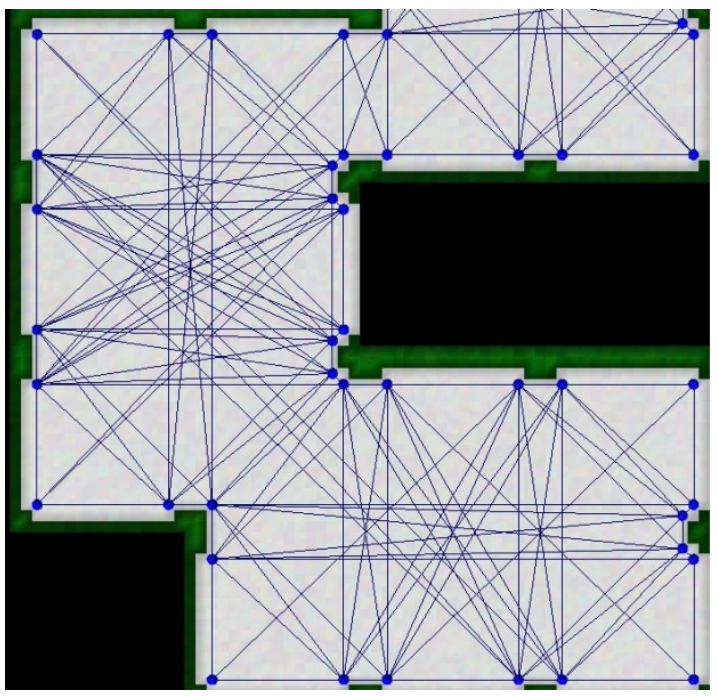

(a) Local Subgoal Graph

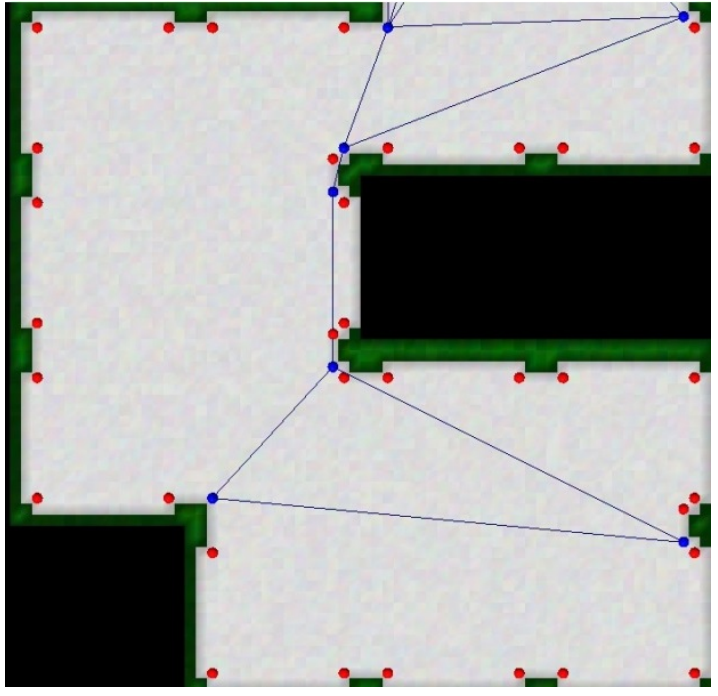

(b) Global Subgoal Graph

Figure 2.8: Basic and Two-Level Subgoals [26]: Blue dots shows nodes in a graph where red dots shows pruned nodes. Edges are represented with blue lines.

Subgoal Graphs is an environment abstraction technique used in fixed location path planning (static path planning). Variations of the technique produced the fastest optimal results for static path planning problem in Grid-Based Path Planning Competition(GPPC) 2013 [26]. Moreover, the required space to create a Subgoal Graph was lower than any other competitors. Subgoal Graphs are a subset of the original search graph and a cost minimal path can be found between any two Subgoal Graph nodes. Since Subgoal Graphs have fewer numbers of edges and nodes, it is faster to search for a cost minimal path in a Subgoal Graph.

A Subgoal Graph consists of subgoals that are nodes in the original search space and corner nodes of the obstacles. Two subgoals are connected with an edge if there is no obstacle or another subgoal lies on the shortest possible path between 


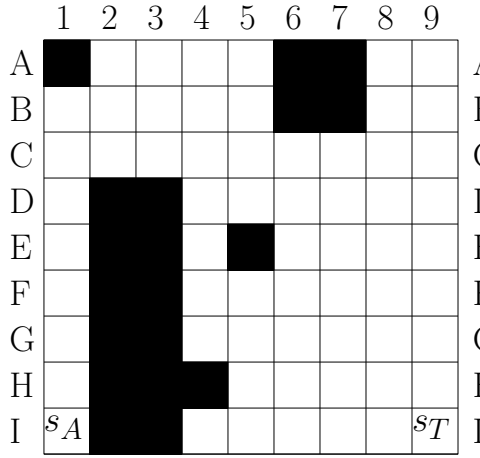

(a) A Search Environment

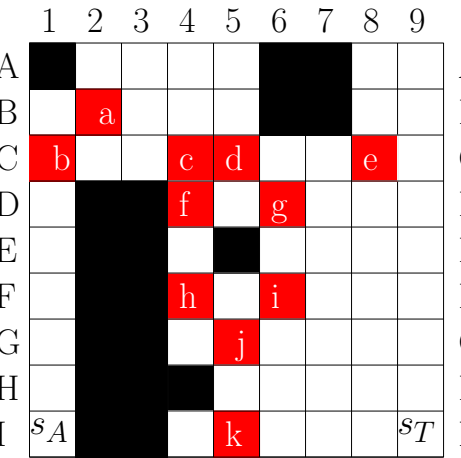

(b) Subgoal Nodes

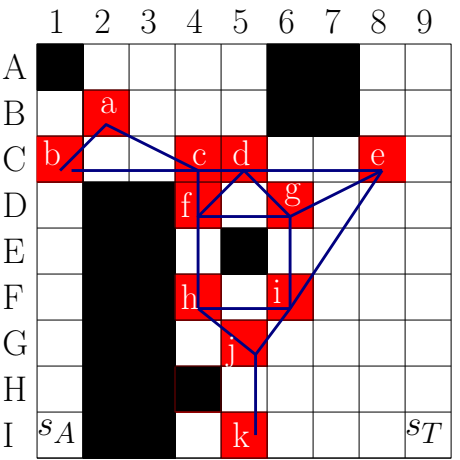

(c) Subgoal Edges
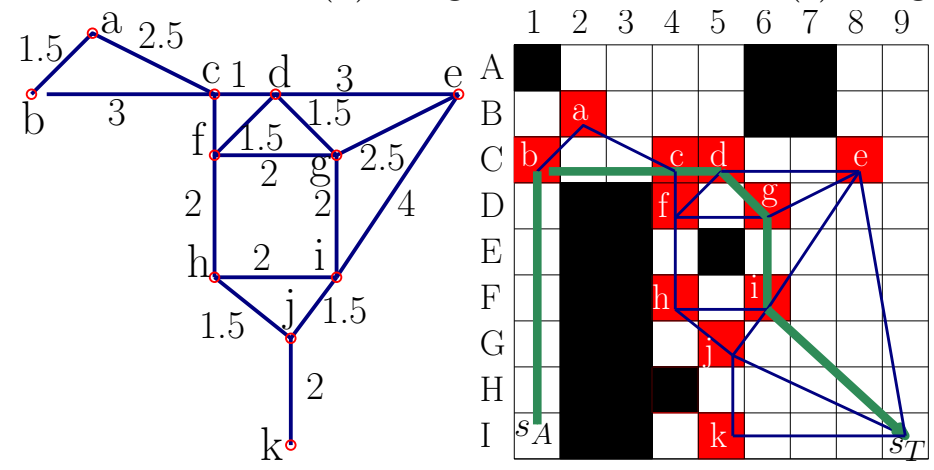

(d) Simple Subgoal Graph (e) Construction of a Path

Figure 2.9: Construction of Simple Subgoal Graphs: Positions of an agent and a target depicted with $s_{A}$ and $s_{T}$, respectively. Black cells are blocked. Red cells are nodes of SSG. Blue lines are edges between nodes of SSG. Numbers indicate edge costs (diagonal cost is 1.5 for simplicity). Green line connecting $s_{A}$ to $s_{T}$ is a cost minimal path.

them. A Subgoal Graph $\left(G_{s}=\left(V_{s}, E_{s}\right)\right)$ is a subset of the original grid graph, where subgoals $\left(V_{s}\right)$ and the connecting edges between subgoals $\left(E_{s}\right)$ are critical and may contribute to the cost minimal paths. This is because if there is an obstacle between two nodes of the original search space, then the cost minimal path between these two nodes has a subgoal. Any unblocked node in the search space can be added to the Subgoal Graph and a cost minimal path between any two subgoals can be found with an $A^{*}$ search. This implies that we can find a cost minimal path between any two nodes of the original search space if they are in the Subgoal Graph 
or they can be added to the Subgoal Graph. An algorithm based on Subgoal Graphs relies on environment abstraction to speed up the cost minimal path searches between stationary locations in an eight connected grid. Instead of searching for a cost minimal path in a given eight connected graph, the method uses a subset of the graph, which is also referred to as Simple Subgoal Graph (SSG). It is faster to search in a Simple Subgoal Graph than in the original graph because the SSG has fewer numbers of edges and nodes. A Simple Subgoal Graph is depicted in Figure 2.8a.

Subgoal Graphs have some similarities to visibility graphs [11]: 1. It is possible to add any node of the original graph to a Subgoal Graph. This means it is possible to connect $s_{A}$ and $s_{T}$ to SG at any time $t$. 2. The edge costs of Subgoal Graphs represent cost minimal paths between corresponding nodes. 3. If $C\left(s, s^{\prime}\right)>H\left(s, s^{\prime}\right)$, where $s$ and $s^{\prime}$ are nodes of SG, there is at least one node $u$ in SG that is on the cost minimal path $\Pi\left(s, s^{\prime}\right)$. 4. If $s$ and $s^{\prime}$ are subgoals and there is an edge of SG that connects them, any $\Pi\left(s, s^{\prime}\right)$, where $C\left(\Pi\left(s, s^{\prime}\right)\right)=H\left(s, s^{\prime}\right)$, is a path. The second, third and fourth resemblances imply that it is possible to find an ordered list of nodes of SG that is on the cost minimal path. Nodes of SG are called subgoals for this reason. This similarity means that it is trivial to transform an ordered list of the subgoals between $s_{A}$ and $s_{T}$, which is referred to as a high level path, to $\Pi\left(s_{A}, s_{T}\right)$, which is referred to as a low level path.

This algorithm creates an SSG only once for a given environment. Algorithms 2.3 and 2.4 show the construction of a Subgoal Graph. First, corner nodes of the obstacles are declared as subgoals and added to $V_{s}$ (Algorithm 2.3, lines 2-7). Second, edges are created between the subgoals if there is a cost minimal path between them with the minimum possible cost and there is not another subgoal in between (Algorithm 


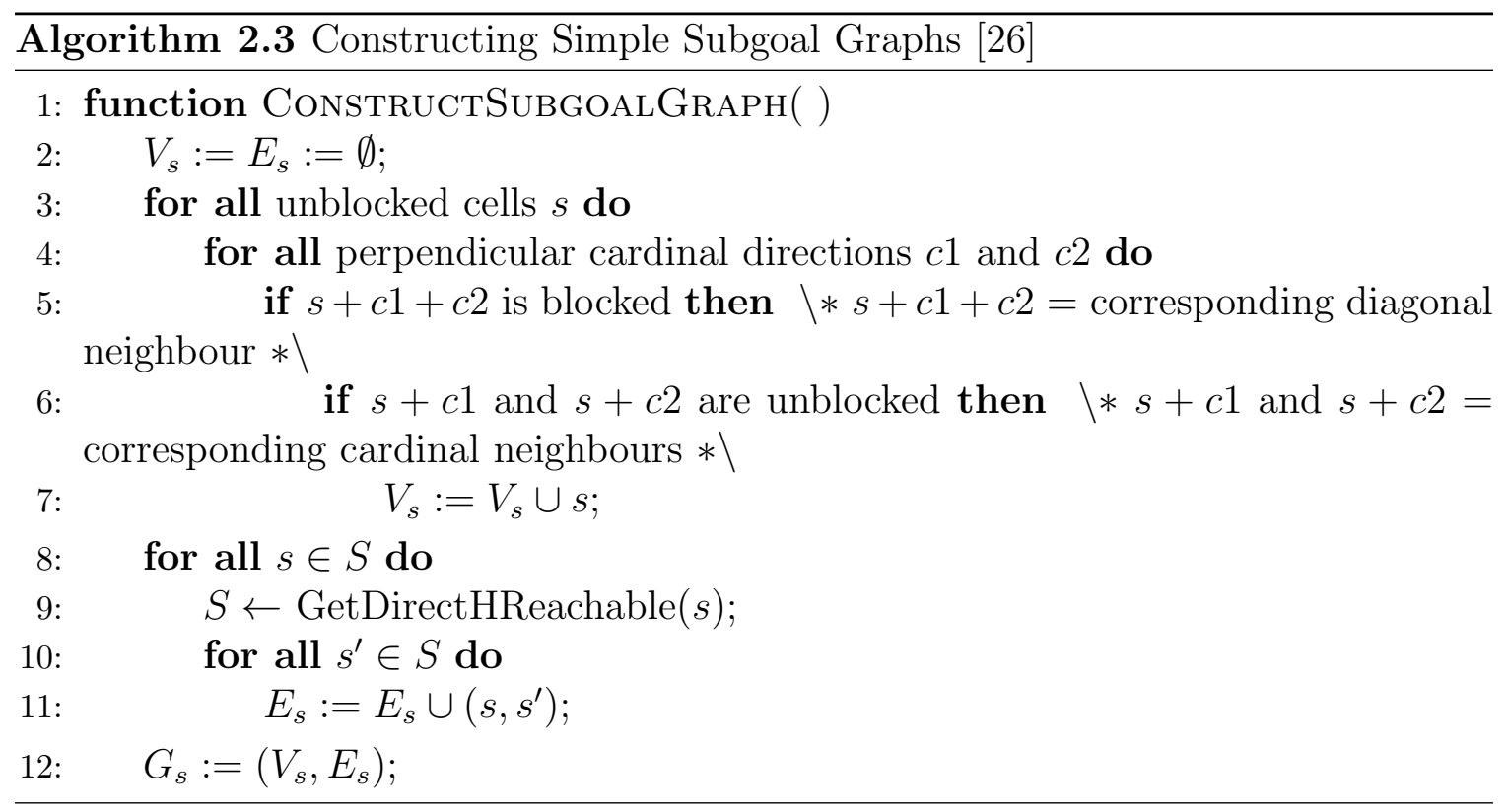

2.3, lines 8-12). The function, GetDirectHReachable scans the environment starting from a given subgoal $s$ and returns all of the subgoals that are reachable from $s$ by following a monotone path (a path that follows only one cardinal and one diagonal direction) in corresponding cardinal and diagonal directions (Algorithm 2.3, line 9). It is important to note that such a path is cost minimal. The function FindPath finds cost minimal paths with Subgoal Graphs (Algorithm 2.4, line 13). If a cost minimal path can not be found by following a monotone path from the agent to the target in corresponding cardinal and diagonal directions (Algorithm 2.4, line 14) then FindAbstractPath is invoked (Algorithm 2.4, line 7). First, the function adds the agent and the target locations to the Subgoal Graph (Algorithm 2.4, lines 8-9) as needed and returns a high level path with the help of an $A^{*}$ search over Subgoal Graph (line 12). A cost minimal path is generated by following a monotone path between subgoals in the high level path (lines 21-22).

The generation of an SSG (Figure 2.9) will be explained. An unblocked node 


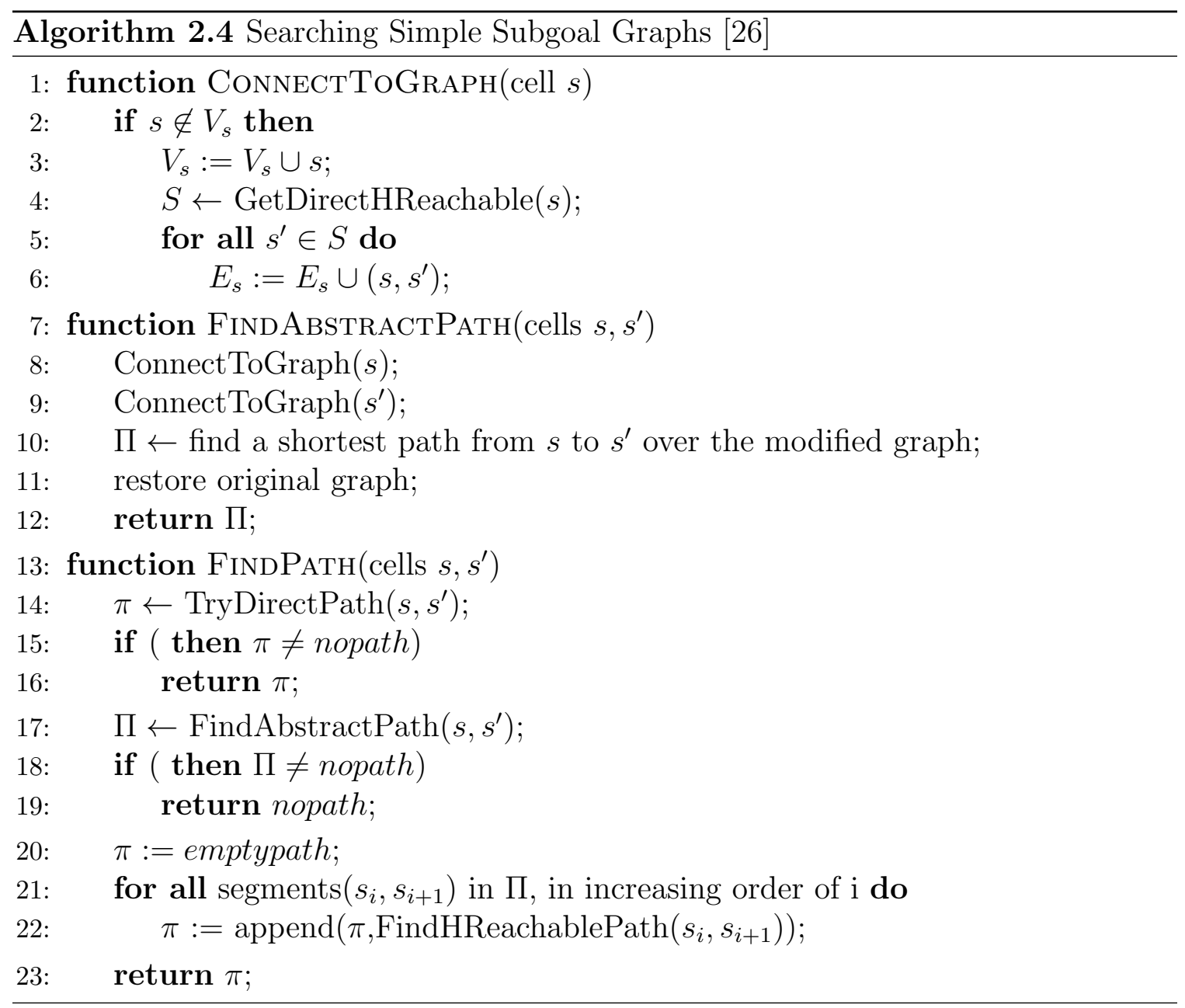

$s$ is marked as a subgoal if at least one of its diagonal neighbours is blocked and corresponding cardinal neighbours are unblocked. Figure 2.9b shows subgoal nodes with red cells. An edge is created between two subgoals $s$ and $s^{\prime}$ if and only if there is a path $\Pi$ such as $C\left(\Pi\left(s, s^{\prime}\right)\right)=H\left(s, s^{\prime}\right)$ and there is no subgoal $s^{\prime \prime}$ such as $C\left(\Pi\left(s, s^{\prime \prime}\right)\right)+C\left(\Pi\left(s^{\prime \prime}, s^{\prime}\right)\right)=C\left(\Pi\left(s, s^{\prime}\right)\right)$ where $s^{\prime} \in S$. For example, in Figure 2.9c, there is no edge connecting subgoals b and d. They are connected via c (as seen in Figure 2.9d) even if there are no obstacles between them. Finding a high level path in an SSG is done by setting $s_{A}$ and $s_{T}$ as subgoals and adding edges using the above 
rule. Figure 2.9e shows connection of $s_{A}$ and $s_{T}$ to an SSG. $s_{A}$ is connected to b since it is the only subgoal that is reachable from $s_{A}$ with heuristic cost. $s_{T}$ is connected to subgoals, e, i, j, k but not to $\mathrm{g}$ since there is a path over i to reach to $\mathrm{g}$. Once done, a high level path can be obtained using an $\mathrm{A}^{*}$ search (Figure 2.9e). Converting the high level path to a low level path is trivial. Please note that there are no obstacles between neighbouring subgoals.

\section{Two-Level Subgoal Graphs}

Hierarchical abstraction can be used to speed up the search by creating layers of Subgoal Graphs in order to accelerate the search. While it is possible to create a hierarchical abstract environment by using n-level subgoals [27], the following will explain Two-Level Subgoal Graphs due to their simplicity when compared to n-level. A Two-Level Subgoal Graph is depicted in Figures 2.8a and 2.8b. Level one is shown in Figure 2.8a and level two is shown in Figure 2.8b.

Nodes in the Two-Level Subgoal Graph, referred to as global subgoals, are a subset of the nodes, or local subgoals, of an SSG. The idea behind Two-Level Subgoals is to eliminate subgoals that are not contributing to a cost minimal path between two neighbours that the subgoal connects.

Global subgoals are selected as follows: First, all of the nodes in an SSG are declared as global subgoals. Second, subgoals are checked for two properties: a subgoal $s$ is declared as local subgoal if 1 . there is a high level path $\Pi$, excluding $s$, such as $C\left(\Pi\left(s^{\prime}, s^{\prime \prime}\right)\right) \leq C\left(s^{\prime}, s\right)+C\left(s, s^{\prime \prime}\right)$ or $2 . C\left(\Pi\left(s^{\prime}, s^{\prime \prime}\right)\right)=H\left(s^{\prime}, s^{\prime \prime}\right), \forall s$ where $s^{\prime}, s^{\prime \prime}$ $\in$ neighbour $(s)$ and $s, s^{\prime}, s^{\prime \prime} \in S$. An edge $\left(s^{\prime}, s^{\prime \prime}\right)$ is created if the second condition is 


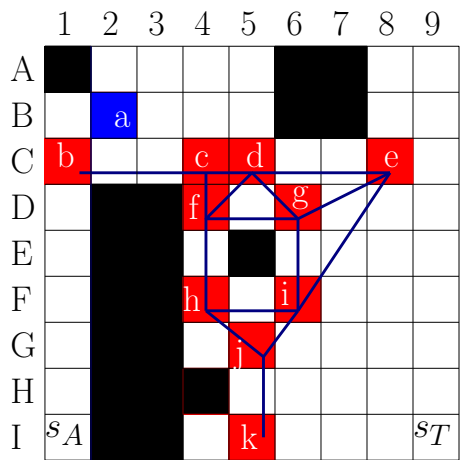

(a) Prune a

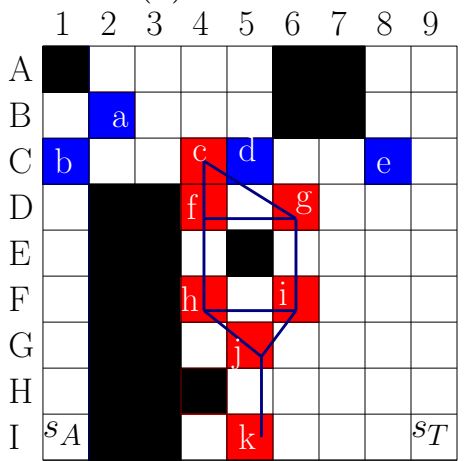

(d) Prune e

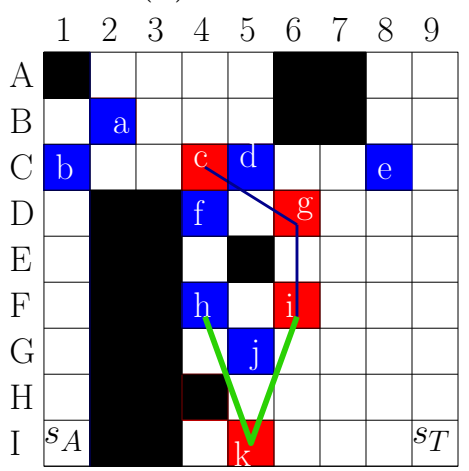

(g) Prune j

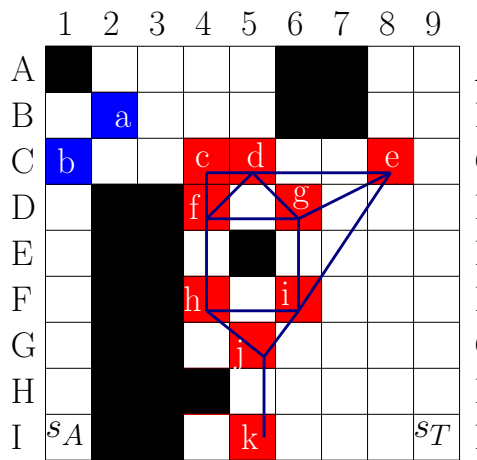

(b) Prune b

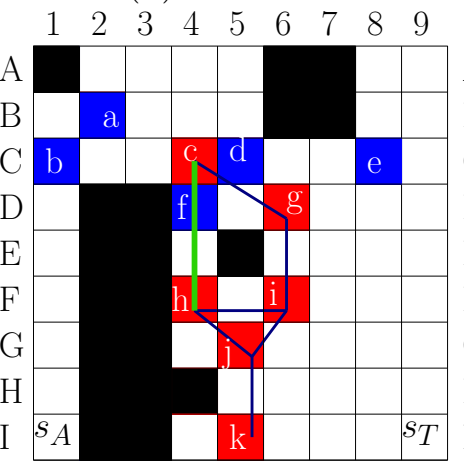

(e) Prune $\mathrm{f}$

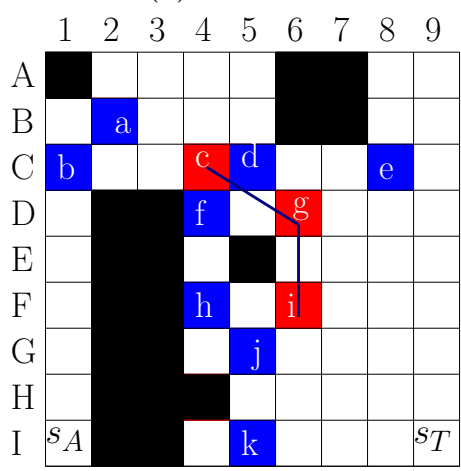

(h) Prune k

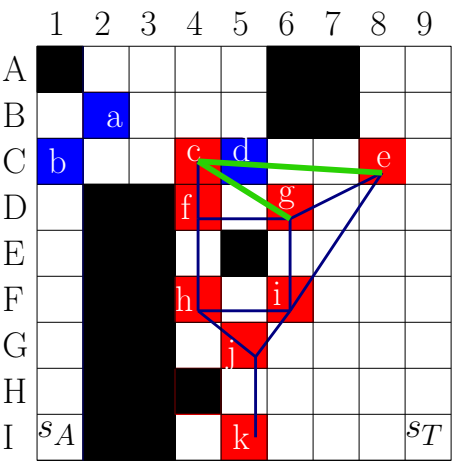

(c) Prune d

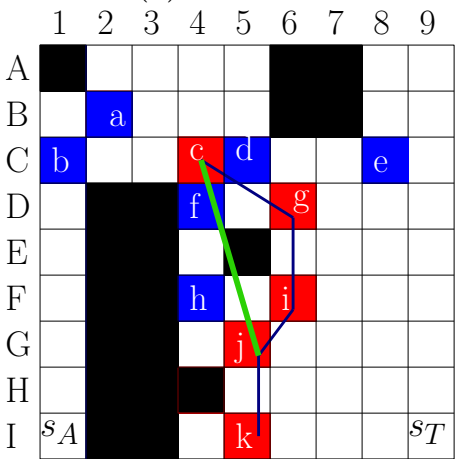

(f) Prune h

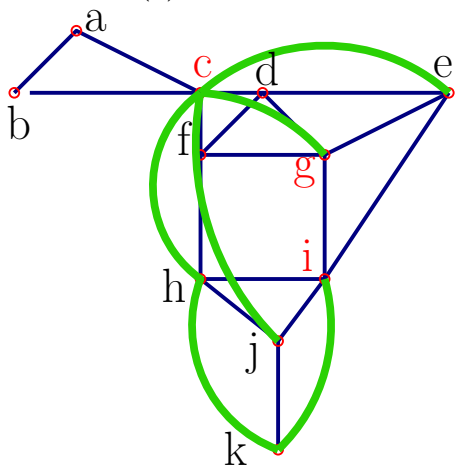

(i) Pruned Graph

Figure 2.10: Construction of a Two-Level Subgoal Graph: Blue cells show pruned nodes whereas red cells show global subgoals. Edges between global subgoals are depicted with blue lines. Newly added edges highlighted with green. The last figure shows the pruned graph. 
satisfied but not the first. An example of this operation can be seen in Figure 2.10.

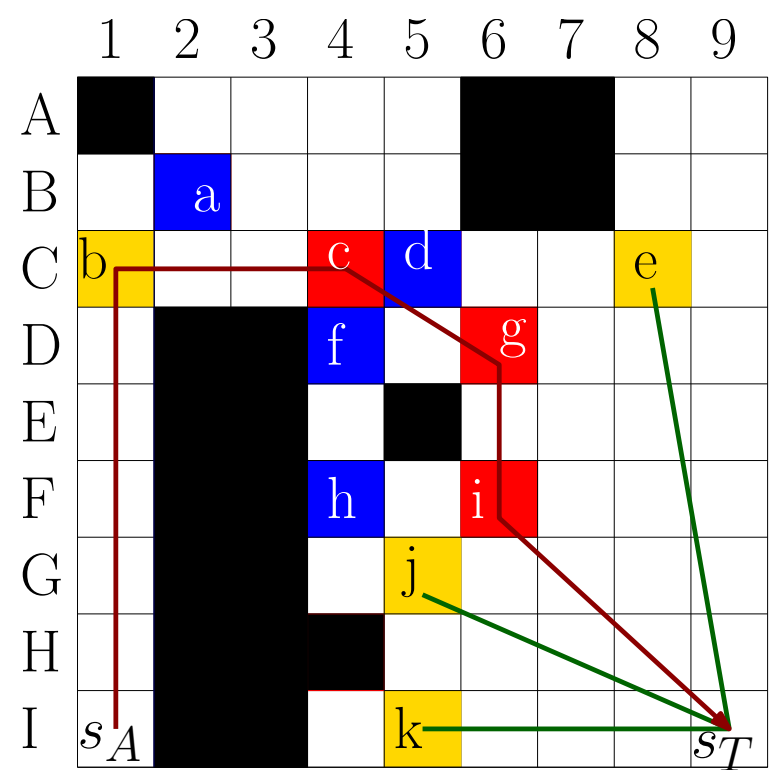

Figure 2.11: Search with a Two-Level Subgoal: $s_{A}$ and $s_{T}$ are connected to the TwoLevel Subgoal Graph. The local nodes that are added after the connection of $s_{A}$ and $s_{T}$ are indicated with yellow cells. Edges between nodes are depicted with green lines whereas final path is highlighted with a red arrow.

A cost minimal path is found with Two-Level Subgoal Graphs as follows: First, $s_{A}$ and $s_{T}$ are added to the Global Subgoal Graph (GSG) if they are not already in GSG or SSG. Second, all neighbours $u$ of $s_{A}$ and $s_{T}$ are declared as global subgoals where $u$ is a local subgoal. Next, $A^{*}$ is used to find a high level path in the Two-Level Subgoal Graph. A directed depth first search (searching with only corresponding cardinal and diagonal direction) is used to construct a low level path between two global subgoals $s, s^{\prime}$. This is because following corresponding cardinal and diagonal directions randomly from one global subgoal to another may not lead to a cost minimal path as it does in SSG. Therefore, transforming a high level path to a low level path in Two-Level Subgoal Graphs may be more costly than that of simple Subgoal Graphs. An example of this operation can be seen in Figure 2.11. 
It is possible to use Two-Level Subgoals for MTS in a repetitive fashion. Such usage of the algorithm is addressed as Repeating Subgoal Graphs (R-Sub).

\subsection{Summary}

This chapter presented background information on the MTS problem and the problem definition. An exhaustive overview of the existing work for MTS is given. In particular, A* algorithm, Subgoal Graphs (SG), Generalized Fringe Retrieving A* (GFRA*), Moving Target Search with Compressed Databases (MtsCopa) are explained with examples. A framework for the MTS problem is also presented to emphasize common points of the algorithms for MTS. 


\section{Chapter 3}

\section{Moving Target Search with Subgoal Graphs}

This chapter discusses the contributions of this thesis. We introduce a new algorithm for MTS. Namely, the Moving Target Search with Subgoal Graphs (MTSub) algorithm is an incremental algorithm that uses environment abstraction to speed up response time without sacrificing optimal solutions. The algorithm builds a Subgoal Graph in order to represent the environment with fewer edges and nodes. Once this has been completed, the algorithm uses the Subgoal Graph throughout the search. MTSub uses the following procedure to find a cost minimal path between any two nodes in the search space through the use of Subgoal Graphs. It first adds the agent and the target locations to the Subgoal Graph (if they are not already added) and finds a cost minimal path between the two. After initial search, MTSub reuses previous information to update the path according to the target's actions. MTSub can find optimal solutions for MTS quickly because it searches in a smaller space, and it uses 
previous information, and does not compute paths from scratch. The novelty of the algorithm is that it reduces the number of times that a cost minimal path to the target is computed. Specifically, it attempts to update the motion of the agent using existing path information.

This chapter is organized as follows: Section 3.1 states the purpose of the suggested methods and what we are trying to achieve. Section 3.2 explains the basic ideas behind the suggested algorithm, namely Moving Target Search with Subgoal Graphs (MTSub). Section 3.3 gives a detailed explanation and proofs for MTSub. Section 3.4 gives examples with figures to simplify the description of MTSub.

\subsection{Objective}

This work aims to introduce better solutions for the Moving Target Search problem. It is possible to compare algorithms for MTS according to a number of criteria. We list some of these below:

1. Quality of the Plan: The quality of a plan is considered better than other plans if it leads an agent to catch a moving target with a lower cost. The cost can be defined by time, energy, the shortest distance or some other related criteria.

2. Applicability: This criterion includes kind and quantity of resources required to use an algorithm for MTS. These requirements are classified below:

Preprocessing Requirements: Space and time requirements of an MTS algorithm in preprocessing are examined under this criterion. 
Run Time Requirements: Space and time requirements of an MTS algorithm during a search are evaluated with this criterion.

This thesis aims to offer better solutions for MTS with respect to the above explained criteria. To achieve this, a novel algorithm is introduced and possible extensions are discussed.

As mentioned previously, we will be introducing a novel algorithm for MTS, namely Moving Target Search with Subgoal Graphs(MTSub). MTSub is an optimal algorithm with respect to our definition in subsection 2.2.1. One of the requirements of MTSub is to have a static 8-connected grid space representation to operate in. This is because it uses an environment abstraction technique. The desired environment representation for MTSub is explained in detail in subsection 2.1.1. Preprocessing and runtime requirements of MTSub compare fairly well against the existing algorithms. MTSub sets itself as state-of-the-art optimal algorithm for MTS when large amounts of preprocessing time and memory are not available.

\subsection{Key Idea}

Two observations about the MTS problem defined in section 2.2 can be made:

I There are more than one cost minimal paths between two locations on a grid and an $\mathrm{A}^{*}$ search spends time to find all the paths with the same cost.

II In most of the cases, a cost minimal path $\Pi\left(s_{A}, s_{T}\right)$ at time $t$ shares many common grid cells with a cost minimal path $\Pi\left(s_{A}, s_{T}\right)$ at time $t-1$. 
The first observation states that the environment has cost-symmetric paths that have the same cost. Thus, an A* search takes additional time to calculate all of the cost minimal paths instead of finding only one of them. To be quicker, MTSub uses Subgoal Graphs to represent a grid environment. Subgoal Graphs contain both critical nodes and the edges connecting those nodes that may contribute to a cost minimal path. An $A^{*}$ search over a Subgoal Graph finds a cost minimal path without doing duplicate searches for symmetric paths. However, a Subgoal Graph may be dense, which increases the search time. MTSub uses a hierarchal abstraction technique, namely Two-Level Subgoal Graphs, to overcome this problem.

The second observation indicates that the information from previous searches can be used to calculate a cost minimal path with fewer resources. Once there is data from previous solutions, MTSub is able to conduct searches faster by using an incremental approach.

The details of the MTSub are given in the next section.

\subsection{Description}

Moving Target Search with Subgoal Graphs(MTSub) is an optimal algorithm that attempts to update the path using knowledge from the previous step. MTSub is different from other incremental MTS algorithms because it uses a Two-Level Subgoal Graph, which is constructed during a preprocessing step.

MTS in known environments is a series of similar path planning problems. However, it is not possible to apply incremental search methods search methods (such as 


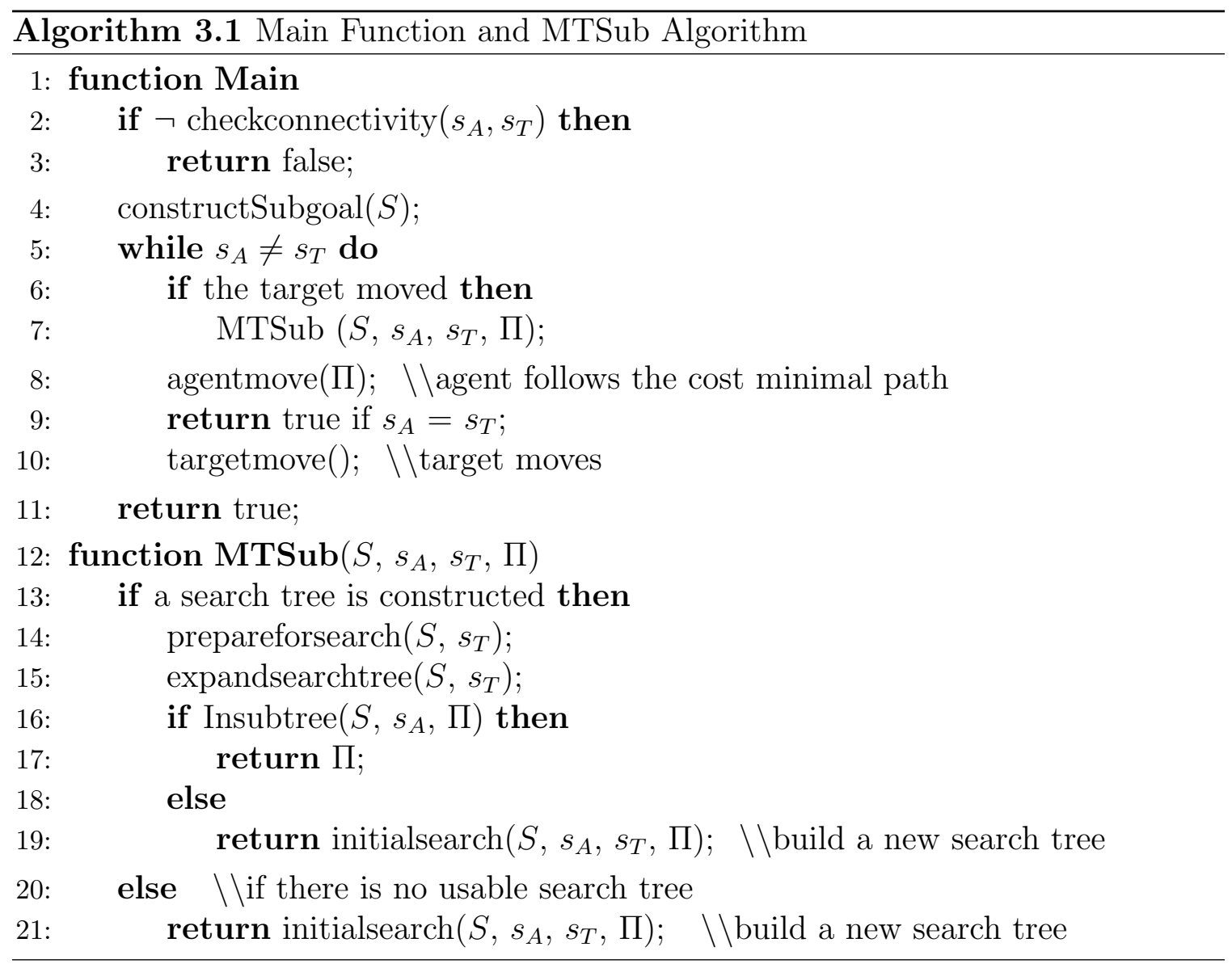

those used in grid graphs) directly to the Two-Level Subgoal Graphs. This is because previous incremental methods for MTS, such as G-FRA* [23], assume that they are given a connected graph. This is the case for grid graphs. That is, any location that an agent or a target can move to is represented with a node. In contrast, only a subset of such locations is represented in Two-Level Subgoals. This implies that the current agent and target locations may not be a part of the Two-Level Subgoal. Therefore, the locations $s_{A}, s_{T}$ and their local neighbours must be connected to the Two-Level Subgoal Graph before initiating a path search, $\Pi\left(s_{A}, s_{T}\right)$, at a time $t$. Unfortunately, inserting new nodes and edges to the Two-Level Subgoal Graph at every time step 
is time consuming (and the search is somewhat longer depending how many nodes and edges were added). In addition, those nodes must be removed after the search in order to preserve the Two-Level Subgoal Graph properties.

MTSub overcomes this problem as follows: First, if there is no search tree then a new tree is constructed after $s_{A}$ and $s_{T}$ are connected to the Two-Level Subgoal Graph. After obtaining a path $\Pi\left(s_{A}, s_{T}\right), s_{T}$ and local neighbours of $s_{T}$ are removed from the search tree and from the Two-Level Subgoal Graph $\left(s_{T}\right.$ is not added or removed if it is already a global subgoal). Note that this operation does not spoil the search tree connectivity as the search tree stops expanding once it reaches $s_{T}$. Thus, by definition of global subgoals there are no nodes in the search tree that are rooted at $s_{T}$. Local neighbours of $s_{T}$ do not contribute to the cost minimal paths between two global nodes. Therefore, they do not connect two global nodes in the search tree and removing them does not violate connectivity of the search tree. Once there is an existing search tree, MTSub does not connect the current $s_{A}$ to the TwoLevel Subgoal but it connects $s_{T}$. Next, MTSub expands the search tree rooted at $\operatorname{position}\left(A, t_{i}\right)$ where $A$ is the agent and $t_{i}$ is the time step when the search tree is initiated. After a cost minimal path $\Pi\left(\operatorname{position}\left(A, t_{i}\right), s_{T}\right)$ is found, MTSub checks if $s_{A}$ is on the path. If it is, MTSub returns a part of the path $\Pi\left(\operatorname{position}\left(A, t_{i}\right), s_{T}\right)$ from $s_{A}$ towards $s_{T}$. Note that the returned path is also a cost minimal path because it is a sub-path of a cost minimal path. If $s_{A}$ is not on the path $\Pi\left(\operatorname{position}\left(A, t_{i}\right), s_{T}\right)$, MTSub connects $s_{A}$ to the Two-Level Subgoal Graph, constructs a new search tree that is rooted at $s_{A}$ from scratch, and finds a path $\Pi\left(s_{A}, s_{T}\right)$. Note that the graph does not grow as we continue the incremental search since we remove $s_{T}$ at the end of every search. Details of the algorithm are explained below with the help of the 
pseudo-code.

Algorithm MTSub checks if the agent and the target are positioned in the same component of the grid graph (line 2). If they are not in the same component, then a path $\Pi\left(s_{A}, s_{T}\right)$ does not exist. Otherwise, $\forall t$ a path $\Pi(\operatorname{position}(A, t), \operatorname{position}(T, t))$ exists. Next, the Two-Level Subgoal Graph is generated (line 4). The agent follows a cost minimal path constructed by MTSub until it reaches the target. If the target moves, the path is updated with the MTSub function (line 7).

MTSub is an algorithm that returns a cost minimal path between $s_{A}$ and $s_{T}$. MTSub executes as follows: If there is no existing search tree, then MTSub calls initialsearch (line 21). The function uses $A^{*}$ to create a search tree over a TwoLevel Subgoal Graph to find a cost minimal path. After the end of the function, a cost minimal path $\Pi\left(s_{A}, s_{T}\right)$ is generated and a reusable search tree rooted at $s_{A}$ is initiated. The root of the tree, which is at position $\left(A, t_{i}\right)$ where $t_{i}$ is the time step when the search tree is built, stays same until a new search tree is constructed.

Lemma 1 The initialsearch function returns a cost minimal path $\Pi\left(s_{A}, s_{T}\right)$.

Proof: A Two-Level Subgoal Graph is connected. The initialsearch function connects $s_{A}$ and $s_{T}$ to the Subgoal Graph. Then, the function uses A* algorithm to search over Two-Level Subgoal Graph to find a path. The function always returns a path $\Pi\left(s_{A}, s_{T}\right)$ since $\mathrm{A}^{*}$ guarantees to find a path in a connected graph.

Based on Theorem 2 in [26] an $A^{*}$ search over a Two-Level Subgoal Graph returns a cost minimal path. The initialsearch uses the exact same methods that are mentioned in Theorem 2 [26]. The heuristic function $H$ that is used by the 
initialsearch obeys the triangle inequality. Octile distance is used as a heuristic. It gives the shortest possible distance between two nodes if we assume that there are no obstacles in the environment. Therefore, it is not possible to find a node $s$ where $H(u, v)>H(u, s)+H(s, v)$ and $u, v, s \in S$. Thus, The initialsearch function returns a cost minimal path $\Pi\left(s_{A}, s_{T}\right)$.

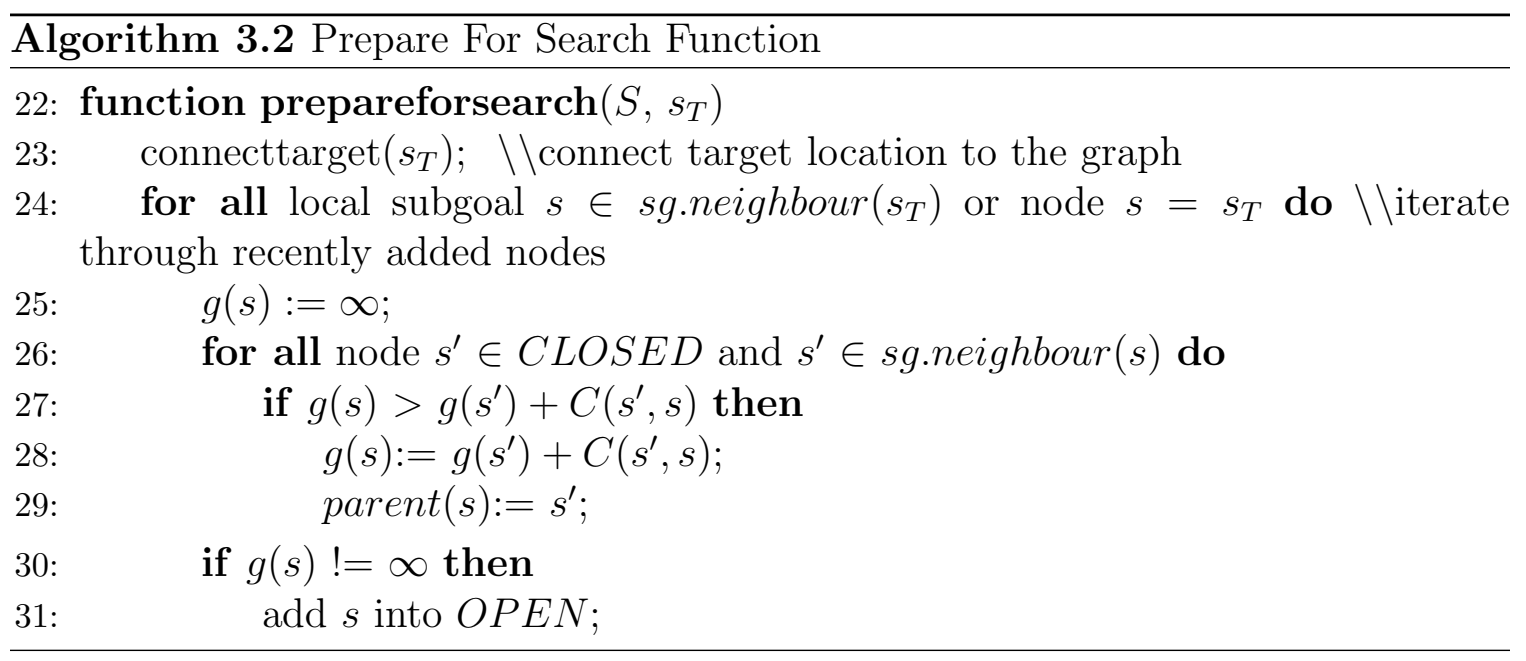

Once MTSub has a search tree, prepareforsearch function is invoked (line 14) as a first step of the incremental search. First, the function connects $s_{T}$ to the graph with corresponding edges (line 23). Next, the function connects $s_{T}$ and the local neighbours of $s_{T}$ (nodes that are newly marked as global subgoals) to the search tree as follows: The function updates parent accordingly (line 29) and the $g$ value of every node $s$ that was recently added to the Two-Level Subgoal Graph by minimizing $g(s)=g\left(s^{\prime}\right)+C\left(s^{\prime}, s\right)$, where $s, s^{\prime} \in S, s^{\prime} \in C L O S E D$ and $s^{\prime} \in \operatorname{sg}$.neighbour(s) (line 28 ). Node $s$ is added to the $O P E N$ list when $g(s) \neq \infty$ (line 31). MTSub calculates $f$ values as $f(s)=g(s)+H\left(s, \operatorname{position}\left(T, t_{i}\right)\right)$ where $s \in S$ and $\operatorname{position}\left(T, t_{i}\right)$ is the location of the target when the search tree is initiated. Note that heuristic values are 
not admissible after the execution of this function because the actual cost of $s(g(s))$ may be smaller than the provided heuristic value $(h(s))$.

Lemma 2 After prepareforsearch function executes, the OPEN list has all the required nodes for a cost minimal path search between the root of the search tree and $s_{T}$.

Proof: Algorithm A* guarantees that if the CLOSED list is not empty, then $O P E N$ consists of nodes $v$ such that $\forall v \in O P E N, \exists u \in C L O S E D$ such that $u \in$ neighbour $(v)$. Therefore, $\forall v \in O P E N \exists u \in C L O S E D \wedge u \in$ neighbour $(v)$ and $u$ minimizes $g(v)=g(u)+C(v, u)$. The function is invoked when a search tree is already in place and therefore the OPEN and the CLOSED lists are already populated. However, the OPEN list may not be complete because the function adds $s_{T}$ and every local subgoal $s$ where $s \in \operatorname{sg}$.neighbour $\left(s_{T}\right)$ to the graph after an $\mathrm{A}^{*}$ search was executed.

To fix this problem, the function prepareforsearch examines every node $s$ that was added to the Two-Level Subgoal Graph. Since $s$ is recently added to the TwoLevel Subgoal Graph, it is not in OPEN or CLOSED. Here the function either finds a node $s^{\prime} \in C L O S E D$ and $s^{\prime} \in$ sg.neighbour $(s)$ that minimizes $g\left(s^{\prime}\right)+h(s)$ or no such node $s^{\prime}$ exists. In the former case, the function sets $g(s)=g\left(s^{\prime}\right)+h(s)$ and parent $(s)=s^{\prime}$ and it inserts $s$ into the $O P E N$ list (If there is more than one $s^{\prime}$, we pick the one that minimizes $f(s)$.). In the latter case $s$ is not reachable from any node in $s^{\prime} \in C L O S E D$ and therefore is not added to the OPEN list. These operations guarantee that recently added nodes are either in $O P E N$ or their parents can be expanded in future iterations of $\mathrm{A}^{*}$. 
Therefore, when prepareforsearch terminates, the $O P E N$ list has all required nodes for a search.

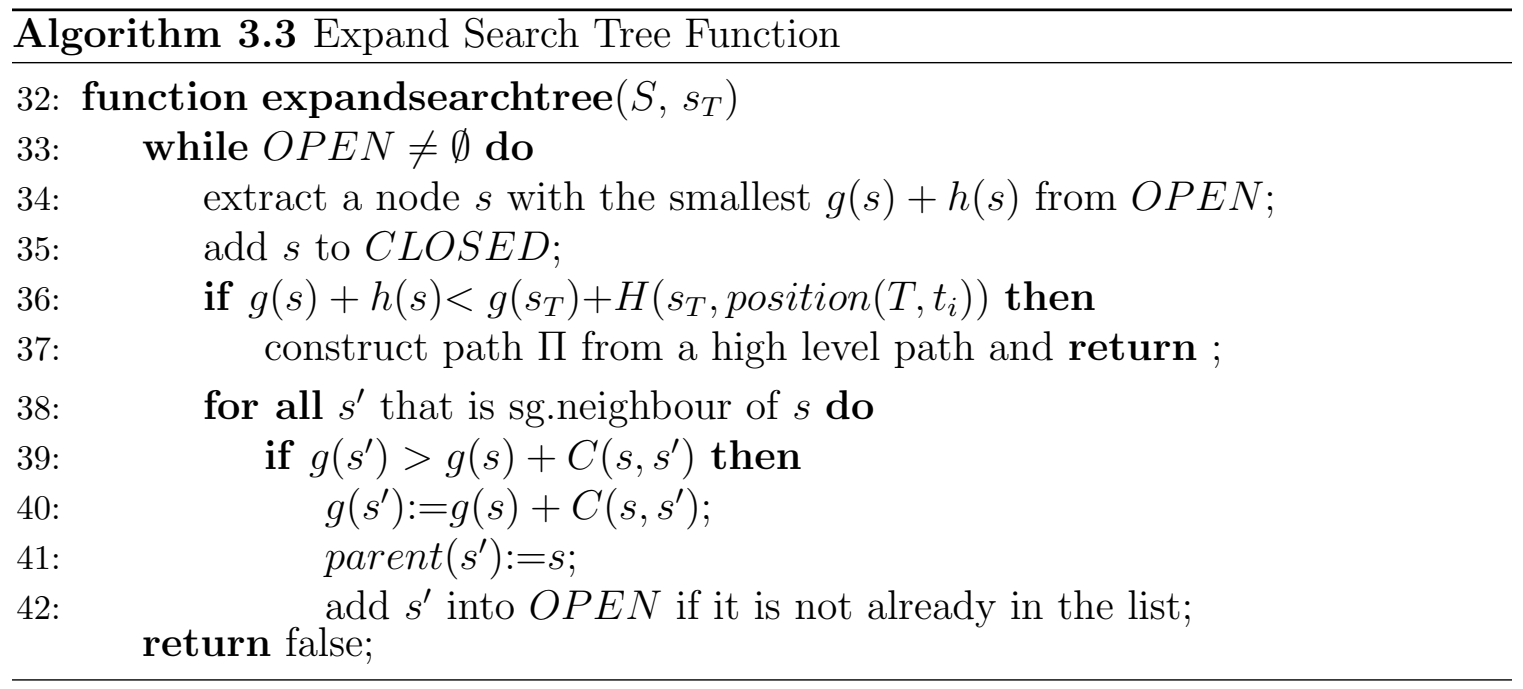

Next, MTSub executes expandsearchtree to find a path between the root of the search tree and $s_{T}$ (line 15). The function builds on the search tree by expanding node $s$ until $f(s)<g\left(s_{T}\right)+H\left(s_{T}, \operatorname{position}\left(T, t_{i}\right)\right)$ where $s$ is a node in the $O P E N$ list with the lowest $f$ value, and position $\left(T, t_{i}\right)$ is the location of the target when the search tree is initiated (line 36$)$. Note that we add $H\left(s_{T}, \operatorname{position}\left(T, t_{i}\right)\right)$ to $g\left(s_{T}\right)$ to make sure that every node that may be on the cost minimal path is expanded even if they are disadvantaged. This is because we do not use admissible heuristics in incremental search iterations. The heuristic value, $h(s)$ can be greater than $H\left(s, s_{T}\right)$ by at most $H\left(s_{T}, \operatorname{position}\left(T, t_{i}\right)\right)$ where $t_{i}$ is the time step at which the search tree was first built. Expanding all nodes in $O P E N$ that may be affected by this difference guarantees that $\mathrm{A}^{*}$ finds a cost minimal path. The root of the existing search tree is $\operatorname{position}\left(A, t_{i}\right)$ where $t_{i}$ is the time step when the search tree is initiated. Please note 
that the calculated $g$ values of the nodes in the search tree are correct because the algorithm expands the tree without changing its root.

Lemma 3 The expandsearchtree function returns a cost minimal path.

Proof: Algorithm $\mathrm{A}^{*}$ does not end as long as there exists a node $s \in O P E N$ such as $g(s)+H\left(s, s_{T}\right)<g\left(s_{T}\right)$ in order to ensure that all possible paths leading to $s_{T}$ were explored. Similarly, the function expandsearchtree does not terminate if there exists a node $s \in O P E N$ such as $g(s)+H\left(s\right.$, position $\left.\left(T, t_{i}\right)\right)<g\left(s_{T}\right)+$ $H\left(s_{T}, \operatorname{position}\left(T, t_{i}\right)\right)$. This equation can be rewritten as $g(s)+H\left(s, \operatorname{position}\left(T, t_{i}\right)\right)$ - $H\left(s_{T}, \operatorname{position}\left(T, t_{i}\right)\right)<g\left(s_{T}\right)$. By the triangle inequality, $H\left(s, \operatorname{position}\left(T, t_{i}\right)\right) \leq$ $H\left(s, s_{T}\right)+H\left(s_{T}, \operatorname{position}\left(T, t_{i}\right)\right)$. Therefore, the function does not terminate if there is a node $s$ in $O P E N$ such as $g(s)+H\left(s, s_{T}\right)<g\left(s_{T}\right)$. Hence, the expandsearchtree function returns a cost minimal path.

After a path is constructed between the root of the tree and $s_{T}$, MTSub checks if $s_{A}$ is on the path with the help of the insubgoal function (line 16). If $s_{A}$ is on the path, a cost minimal path $\Pi\left(s_{A}, s_{T}\right)$ is generated and returned. Otherwise, a new search tree is constructed with initialsearch function (line 19).

Lemma 4 The insubgoal function returns a cost minimal path.

Proof: The function insubgoal is only executed if there is a cost minimal path, $\Pi\left(\operatorname{position}\left(A, t_{i}\right), s_{T}\right)$ where $t_{i}$ is the time when search tree is built. The function returns a sub-path $\Pi\left(s_{A}, s_{T}\right)$ if both locations are on the path, $\Pi\left(\right.$ position $\left.\left(A, t_{i}\right), s_{T}\right)$. 
Any sub-path between two nodes of a cost minimal path is also a cost minimal path. Therefore, the returned path is a cost minimal path.

Theorem 3.1 MTSub finds a cost minimal path $\Pi\left(s_{A}, s_{T}\right)$.

Proof: MTSub returns paths that were computed by either initialsearch or insubgoal functions. Both functions return a path, each of which are cost minimal as shown in Lemmas 1 and 4. Therefore, at each step MTSub returns cost minimal paths between current positions of the target and the agent.

Theorem 3.2 The position of an agent that is following a path generated by MTSub is on the optimal route at any time $t_{i}$.

Proof: According to Theorem 3.1, MTSub generates optimal paths between current locations of the agent and the target at $\forall t_{i}$. Thus, the position of the agent, position $\left(s_{A}, t_{i}\right)$, is on the cost minimal path $\Pi\left(\operatorname{position}\left(s_{A}, t_{i-1}\right), \operatorname{position}\left(s_{T}, t_{i-1}\right)\right)$. Therefore, the agent is on the optimal route.

It is possible to use early termination techniques to find cost minimal paths to speed up the incremental search.

The function quickpath which is introduced with Subgoal Graphs [26], checks if it is possible to find a path $\Pi\left(s_{A}, s_{T}\right)$ where $C\left(\Pi\left(s_{A}, s_{T}\right)\right)=H\left(s_{A}, s_{T}\right)$. The function tries to build such a path by starting from $s_{A}$ and following the corresponding cardinal and 
diagonal nodes towards $s_{T}$. If an obstacle is encountered along the path, quickpath returns false. Otherwise, a cost minimal path is returned. We use this method at the beginning of the MTSub function to terminate early.

If there is an existing search tree, the easypath function is used to terminate early before the prepareforsearch function. This function checks if $s_{T}$ is on the existing cost minimal path. If it is, we use the same path because any sub-path of a cost minimal path is also a cost minimal path.

\subsection{Example}

In this section, two sets of examples are provided and used to explain MTSub further. The first set of examples demonstrates the early termination techniques that are used in MTSub. The second set of examples explains how MTSub finds a path in an incremental fashion and when MTSub builds a search tree from scratch.

Early termination methods that are used by MTSub, namely quickpath and easypath, are demonstrated in Figure 3.2.

The quickpath method (shown in Figure 3.2 above), tries to build a path by starting from $s_{A}$ and following the corresponding cardinal and diagonal nodes towards $s_{T}$. In the example shown in Figure 3.2a, such a path is found. Note that a path may not be found by quickpath as it constructs a path by arbitrarily choosing corresponding moves. Figure $3.2 \mathrm{~b}$ shows a case where quickpath fails to find a path between the same locations. The illegal move is highlighted with blue.

Figures $3.2 \mathrm{c}$ and $3.2 \mathrm{~d}$ show the locations of an agent and a target at time $t$ and 


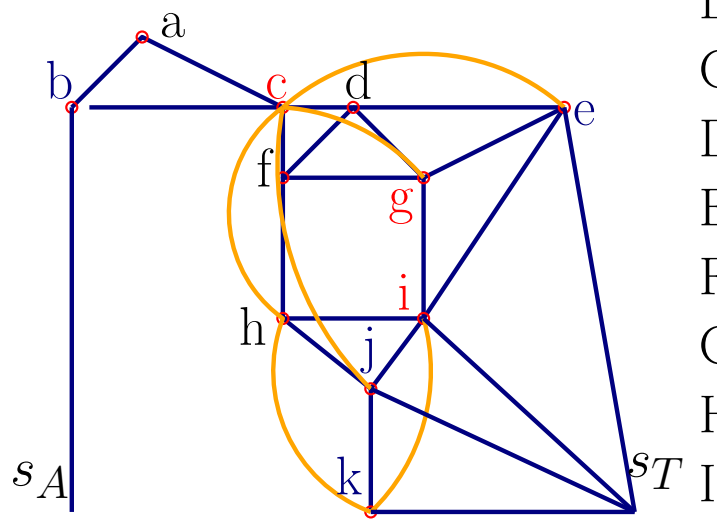

(a) Two-Level Subgoal with $s_{A}$ and $s_{T}$ $\left(t_{0}\right)$

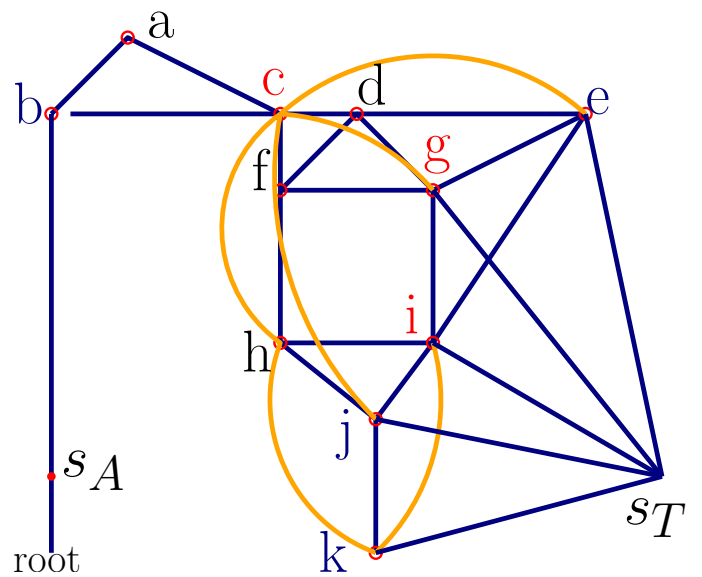

(c) Two-Level Subgoal with $s_{A}$ and $s_{T}$ $\left(t_{1}\right)$

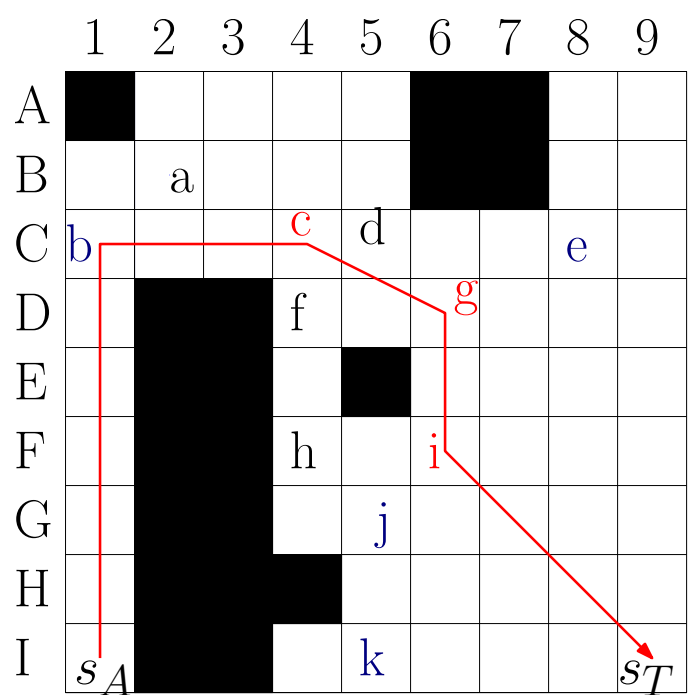

(b) initial search $\left(t_{0}\right)$

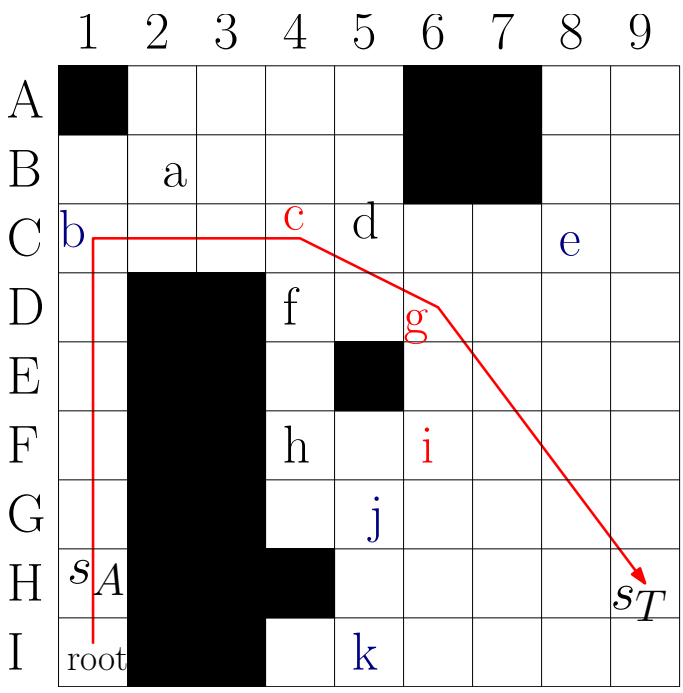

(d) incremental search $\left(t_{1}\right)$

Figure 3.1: Execution of MTSub - Incremental Search: Locations of $s_{A}, s_{T}$ and root of the search tree are depicted in the figures. Black grid cells are obstacles. The figure uses red to indicate global subgoals and the blue to show local subgoals (those that are recently added to the Two-Level Subgoal Graph). Black labels indicate pruned nodes. Blue lines show subgoal edges while orange lines show the edges added after the pruning. Paths are highlighted with red arrows. 


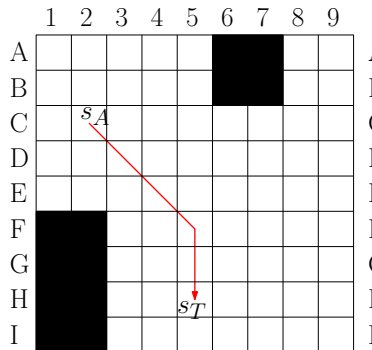

(a) quickpath (success)

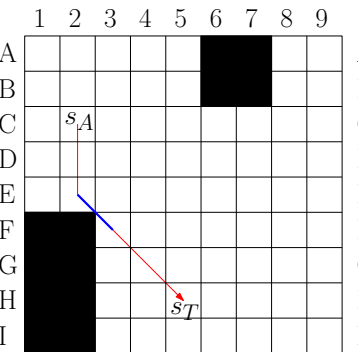

(b) quickpath (failure)

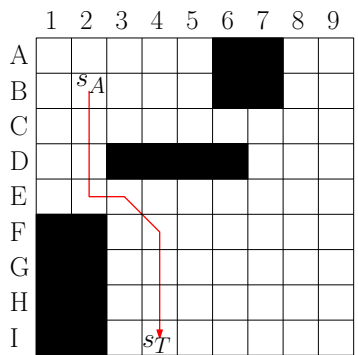

(c) the path before easypath $(t)$

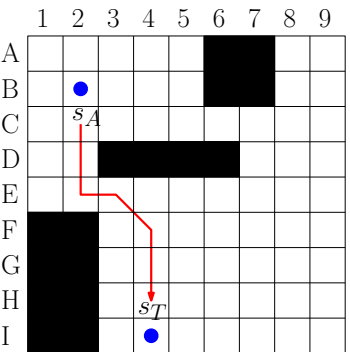

(d) easypath $(t+1)$

Figure 3.2: Early Termination Methods: Arrows are used to indicate cost minimal paths. Black cells represent blocked nodes. Blue cells are previous locations of the agent and the target.

$t+1$. At time $t+1$, easypath decides that the existing path is still usable since $s_{T}$ is still on the path (Figure 3.2d). The previous locations of the agent and the target (grid cells B2 and I4) are highlighted with green dots.

Figure 3.1 shows a case where MTSub finds a path with incremental search. The steps taken to do so are as follows:

Time Step $t_{0}$ : The initial positions of the agent and the target are I4 and I9, respectively. Figure 3.1a shows the Two-Level Subgoal graph at time step $t_{0}$. The subgoals $b, e, j$ and $k$ are not global subgoals but they are added to the TwoLevel Subgoal graph in the process of connecting $s_{A}$ and $s_{T}$ to the graph. Since there is no existing information at time step $t_{0}$, MTSub invokes initialsearch. Figure $3.1 \mathrm{~b}$ shows the path, $\Pi\left(s_{A}, s_{T}\right)$, found by MTSub.

Time Step $t_{1}$ : The agent followed the path and moved to H1 while the target moved to H9. Figure 3.1c depicts the Two-Level Subgoal graph at time step $t_{1}$. Note that there is a new edge between $s_{T}$ and $g$. MTSub doesn't connect $s_{A}$ to the graph since there is a search tree. MTSub then invokes prepareforsearch to 
update the search tree according to changes. The subgoals $j, i, g$ and $i$ are added to $O P E N$. The function, expandsearchtree then finds a path that is different from the previous path. The subgoal $i$ is no longer on the path since there is a shorter path between $\mathrm{g}$ and $s_{T}$. However, $s_{A}$ is still on the path $\Pi\left(\right.$ root,$\left.s_{T}\right)$. There is therefore no need to build a new search tree and the agent follows the sub-path $\Pi\left(s_{A}, s_{T}\right)$. Figure $3.1 \mathrm{~d}$ shows the path $\Pi\left(s_{A}, s_{T}\right)$.

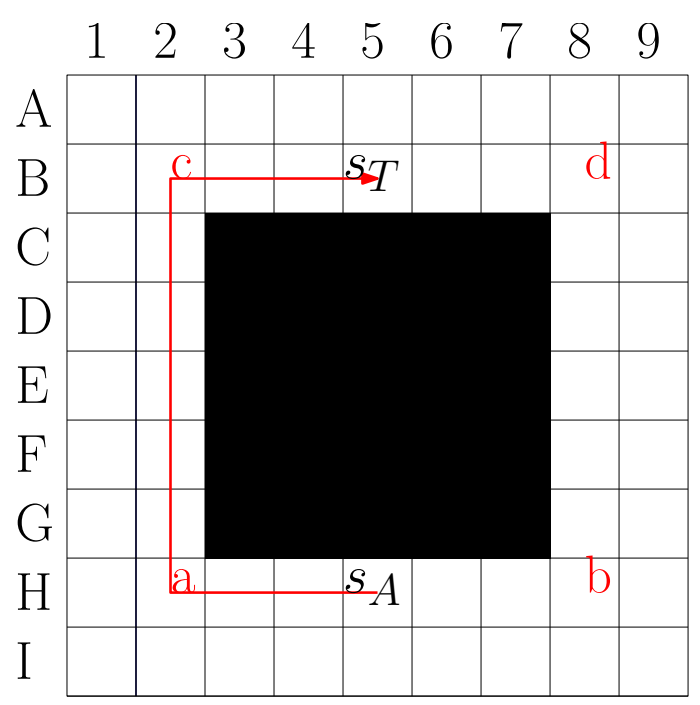

(a) initial search $\left(t_{0}\right)$

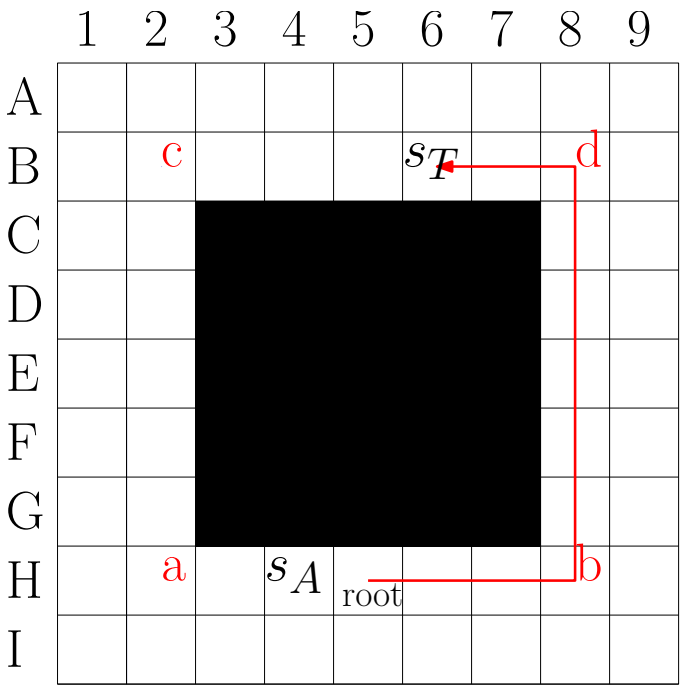

(b) $s_{A}$ is not on $\Pi\left(\operatorname{position}\left(A, t_{0}\right), s_{T}\right)$ at $t_{1}$

Figure 3.3: Execution of MTSub - Rebuilding a Search Tree: Locations of $s_{A}, s_{T}$ and root of the search tree are depicted in the figures. Red labels are used to show the global subgoals.

Figure 3.3 shows a case where incremental search fails and MTSub builds a new search tree:

Time Step $t_{0}$ : The initial positions of the agent and the target are H5 and B5 respectively. All of the subgoals are in the Two-Level Subgoal graph. Since there is no existing information at time step $t_{0}$, MTSub invokes initialsearch. Figure 
3.3a shows the path, $\Pi\left(s_{A}, s_{T}\right)$, found by MTSub.

Time Step $t_{1}$ : The agent followed the path and moved to $\mathrm{H} 4$ while the target moved to B6. Figure 3.3b highlights the path, $\Pi\left(\right.$ root,$\left.s_{T}\right)$, found after the execution of prepareforsearch and expandsearchtree. Unfortunately, $s_{A}$ is not on the path $\Pi\left(\right.$ root,$\left.s_{T}\right)$. Thus, MTSub invokes initialsearch to build a search tree from scratch and find a path, $\Pi\left(s_{A}, s_{T}\right)$.

\subsection{Summary}

This chapter presents the newly developed algorithm MTSub. MTSub uses environment abstraction and incremental search to speed up response time without sacrificing quality of the resulting path. The novelty of the algorithm is the ability to significantly reduce the number of times that a cost minimal path is calculated. Moreover, the algorithm can use its existing information of the path and search space to determine the next move of the agent. 


\section{Chapter 4}

\section{Experimental Results and Analysis}

This chapter presents an empirical evaluation of MTSub and its extensions. Section 4.1 states the objective of the experiments. Section 4.2 explains experimental setup. In section 4.3 we present and discuss the results of various experiments.

\subsection{Objective and Testing Methodology}

The objective of the following experiments is to demonstrate if the suggested algorithm, namely MTSub, and its extensions achieved the objectives set out in Section 3.1 .

We compare MTSub against two algorithms for MTS. These are G-FRA*, which is an incremental algorithm, and MtsCopa, which uses a preprocessing phase to compute a path between any two nodes $s, s^{\prime} \in S$ and stores the results in a database. A previous paper [13] shows that the performance of FRA* and G-FRA* is similar 
and that they have the smallest average run times among optimal algorithms that do not use preprocessing. Thus, we only compare G-FRA* against MTSub in the incremental algorithm category. MtsCopa has the shortest run times [9]. We include MtsCopa in the experiments in order to demonstrate the efficiency of MTSub against an algorithm that exploits preprocessing. We also compared MTSub with algorithms for fixed location path planning, $A^{*}$ and Two-Level Subgoal Graphs, to demonstrate the benefits of incremental search and environment abstraction. We use such algorithms repetitively to find a path $\Pi\left(s_{A}, s_{T}\right)$ and solve the MTS problem. We refer to them as Repeating A* (R-A*) and Repeating Two-Level Subgoal Graphs (R-Sub).

The experiments were conducted as follows: First, a predefined set of maps were selected. The size of a graph and positions of obstacles were determined by a map. Second, scenarios were created which included initial locations of the agent and the target on the graph and target motion. The target route was defined by the target movement strategy (the routes were stored and used by all algorithms). Since the agent and the target move with the same speed, the agent may not catch the target. To prevent the target from running infinitely, we move the target at every time $t_{n}$ if its location at time $t_{n-1}$ is not occupied by the agent at time $t_{n}$. The agent path was determined by the competing algorithms at run time. An experiment ends when $s_{A}$ at time $t_{n}$ is equal to $s_{T}$ at time $t_{n-1}$ and the target cannot move.

\subsection{The Experimental Setups}

This section reviews the general aspects of the experimental setup as follows. 


\subsubsection{Algorithm Implementations}

Comparing algorithms based on their run time performance requires careful implementation of each of the algorithms. In this research we used the original authors' implementations of the competing algorithms in order to avoid any potential bias. We assumed that the authors' implementation of the algorithm is efficient because runtime is a commonly used measure to compare MTS algorithms in the literature $[9,28,29]$. We obtained the implementations of Subgoal Graphs and MTSCopa from the Grid Based Path-Planning Competition (GPPC) repository [30]. GPPC provides a common library (HOG [31]) that all competing algorithms are supposed to use. Author's of G-FRA* algorithm, which was not in the GPPC repository, provided us with a C implementation. The G-FRA* was linked with the HOG library in order to be comparable with the competing algorithms. Last, MTSub also used the HOG library in order to avoid any low level enhancements of basic data structures such as priority queues.

This approach provides an equal coding environment and therefore makes the runtime comparison of the algorithms more reliable. We would like to note that none of the compared algorithms required access to virtual memory. The experiments were executed on a $2.50 \mathrm{GHz}$ Linux machine with 6GB of RAM.

\subsubsection{Map Domains}

We conducted experiments using different map classes to observe the effects of the environment on the algorithm performances. All maps used in the experiments are retrieved from Nathan Sturtevant's path finding repository [32]. Characteristics of 


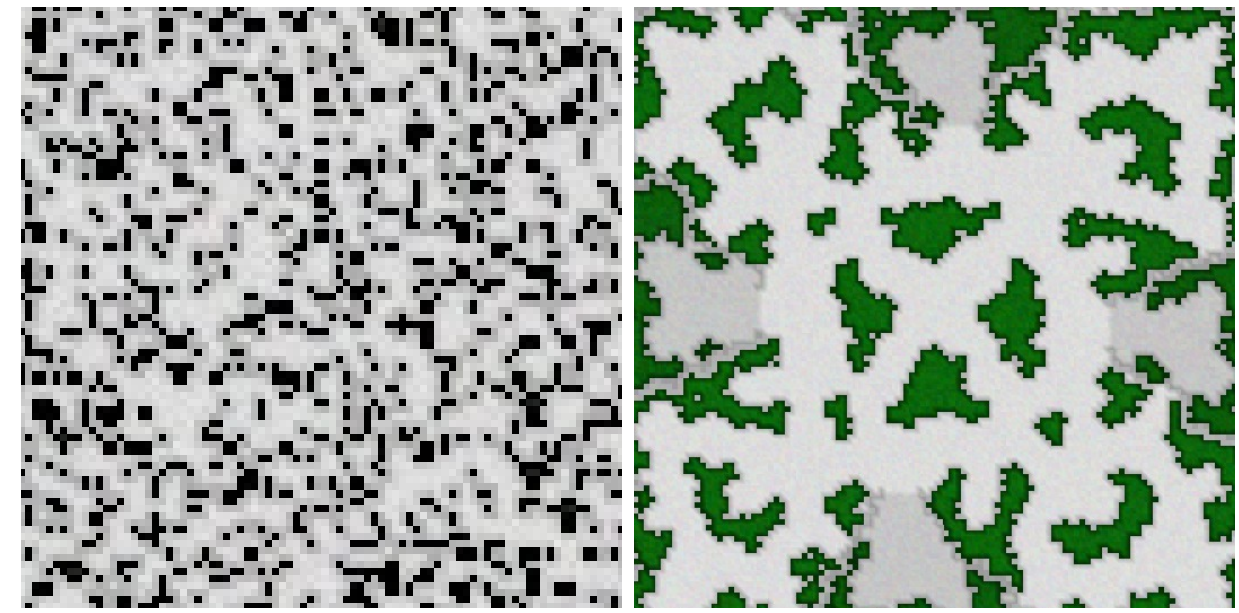

(a) Random Map

(b) Constructed Map

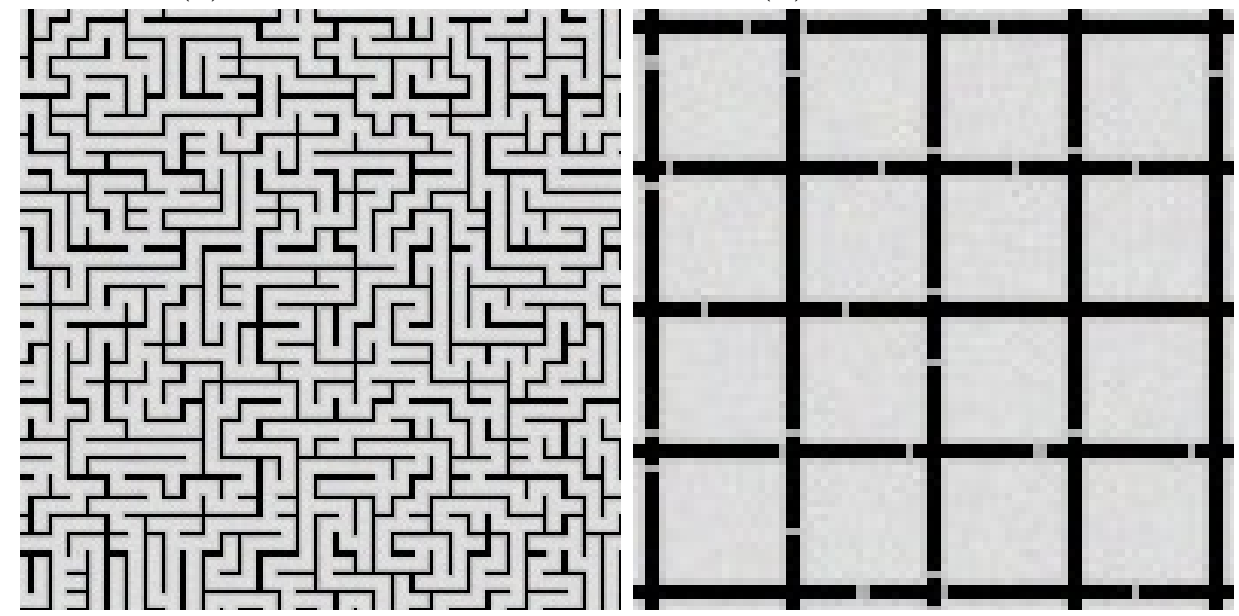

(c) Maze Map

(d) Room Map

Figure 4.1: Map Classes 


\begin{tabular}{c|ccc} 
Map Set & Average size of $S$ & Max. Path Cost & Heuristic Accuracy \\
\hline Constructed(BG512) & $73,930(\% 28)$ & 693.0 & 0.76 \\
Constructed(WCIII512) & $90,910(\% 34)$ & 674.0 & 0.84 \\
\hline Maze-2 & $174,517(\% 66)$ & $5,791.5$ & 0.24 \\
\hline Random-20 & $209,255(\% 80)$ & 816.6 & 0.95 \\
\hline Room-16 & $231,263(\% 88)$ & 863.8 & 0.83 \\
\hline
\end{tabular}

Table 4.1: Map Class Characteristics [32]: "Average size of $S$ " is the average number of free grid cells. "Max. Path Cost" is the maximum cost of a path between any two locations on the map. "Heuristic Accuracy" is a ratio of estimated heuristic values to optimal costs for the selected paths.

the map classes are shown in Table 4.1 and visuals of maps from each class can be seen in Figure 4.1. "Average size of $S$ " is shows the obstacle density in an environment. "Max. Path Cost" is an estimated average for most costly path. "Heuristic Accuracy" is an estimated average value for user provided heuristics (octile distance in this case). Lower values indicate that the heuristics are usually misleading. The four map domains are discussed further below.

\section{Constructed Maps}

Constructed maps have obstacles and free spaces similar to the real world. Constructed Maps are video game maps that are generated to simulate real world environments. We used Constructed Maps from popular video games such as Warcraft III and Baldur's Gate (Figure 4.1b).

As shown in Table 4.1, Constructed maps have the smallest number of nodes in $S$ among map classes. Maximum path cost of the class is also the smallest of all. Heuristic accuracy of Constructed Maps are less than Random and Room maps, but 
significantly greater than Maze Maps.

These maps are made for simulating real life environments. We used the Constructed Map class to observe the performance of the competing algorithms in both video games and real life robotics applications.

\section{Random Maps}

Random Maps have many small sized obstacles scattered across the map. Random Maps are created by adding obstacles in randomly selected nodes (Figure 4.1a). Twenty percent of the grid cells are blocked in Random Maps.

As shown in Table 4.1, Random maps have the highest heuristic accuracy. Average size of $S$ is also high in this map class. Maximum path cost is the second lowest.

High heuristic accuracy and the chaotic fashion of Random Maps make them particularly interesting. Because $A^{*}$ search is expected to run faster with more accurate heuristics and irregularities found in these kinds of maps decreases the effectiveness of the environment abstraction techniques such as Subgoal Graphs. We use this map class for observing the effects of such parameters on the performances of the algorithms.

\section{Maze Maps}

Maze Maps have obstacles that create a complex and confusing series of paths. Maze Maps are created using narrow corridors and walls to form a maze as the class name would suggest (Figure 4.1c). 
As shown in Table 4.1, Maze maps have the lowest heuristic accuracy and highest maximum path cost. The average size of $S$ is less than Room and Random Maps but higher than Constructed Maps.

We used Maze Maps in our experiments to observe the effects of misleading heuristics on the competing algorithms.

\section{Room Maps}

Room Maps have rooms that allow access to other rooms through a small number of unblocked cells (Figure 4.1d). To create Room Maps, 16 x 16 sized rooms are created and randomly selected blocked nodes are made unblocked to create openings between the rooms.

As shown in Table 4.1, Room maps have the highest average size of $S$ and second highest maximum path cost. Heuristic accuracy of the map class is close to that of Constructed Maps.

Room Maps are particularly good for environment abstraction since access to large free areas are only through small openings. We used this map class to observe environment effects on map abstraction techniques.

\subsubsection{Behaviour of Targets}

We used two strategies for the targets in our experiments:

I Random Movement: A target moves to a randomly selected unblocked neighbouring grid cell at any time $t$. This strategy makes future movements of the 
target unpredictable.

II Way-Point Following: A target moves on a cost minimal path to reach a randomly selected unblocked grid cell (way-point). If a target can reach a way-point before an agent meets with it, a new way-point is created. This strategy is closer to real-life scenarios where a target moves according to its own agenda disregarding a pursuing agent.

\subsubsection{Measures}

We developed a number of experiments so that we could further understand the various aspects of the competing algorithms. These experiments exposed the following characteristics of the algorithms.

I Quality of the Plan: We report the needed number of time steps (measured with t) by an agent to catch a target to measure the quality of a plan. Both an agent and a target move at the same speed. We used this measure because it is only affected by the agent's strategy.

II Applicability: The measures that we used to inspect preprocessing and run time requirements of the competing algorithms are listed below:

Preprocessing Requirements: For the competing algorithms that are exploiting preprocessing, we reported used memory and elapsed CPU time to measure space and time requirements, respectively. Memory is used because it is a direct indicator of space requirements, while CPU time (for a single CPU) is used to eliminate the effects of operating systems and multi-threading on the elapsed 
time.

RunTime Requirements: For run time, we reported used memory and elapsed CPU time (response time). We did not use parallel computing. The reported times are for sequential runs. Maximum and average response times are given to inspect time requirements better. Used Memory is chosen to measure space requirements since it is a direct indicator.

\subsection{Results}

In this section results of the experiments are presented in three subsections. In each subsection, specific experiments are discussed to provide the reader with a better understanding of how MTSub compares against the existing algorithms for MTS and how MTSub's extensions.

\subsubsection{Comparison with Dynamic Algorithms for MTS}

This section presents our experimental results of comparing MTSub against the dynamic competing algorithms. Results of the experiments, that compare MTSub against dynamic MTS algorithms with a random moving target, are depicted in Table 4.2. Figure $4.2 \mathrm{a}$ visualizes the results in Table 4.2 .

One of the incremental algorithms, G-FRA* has a shorter average runtime than that of R-A*. However, R-A* has a shorter maximum time than that of G-FRA* in all the map domains. These results indicate that repairing a search tree during runtime increases maximum time spent in a search step even if it decreases average 


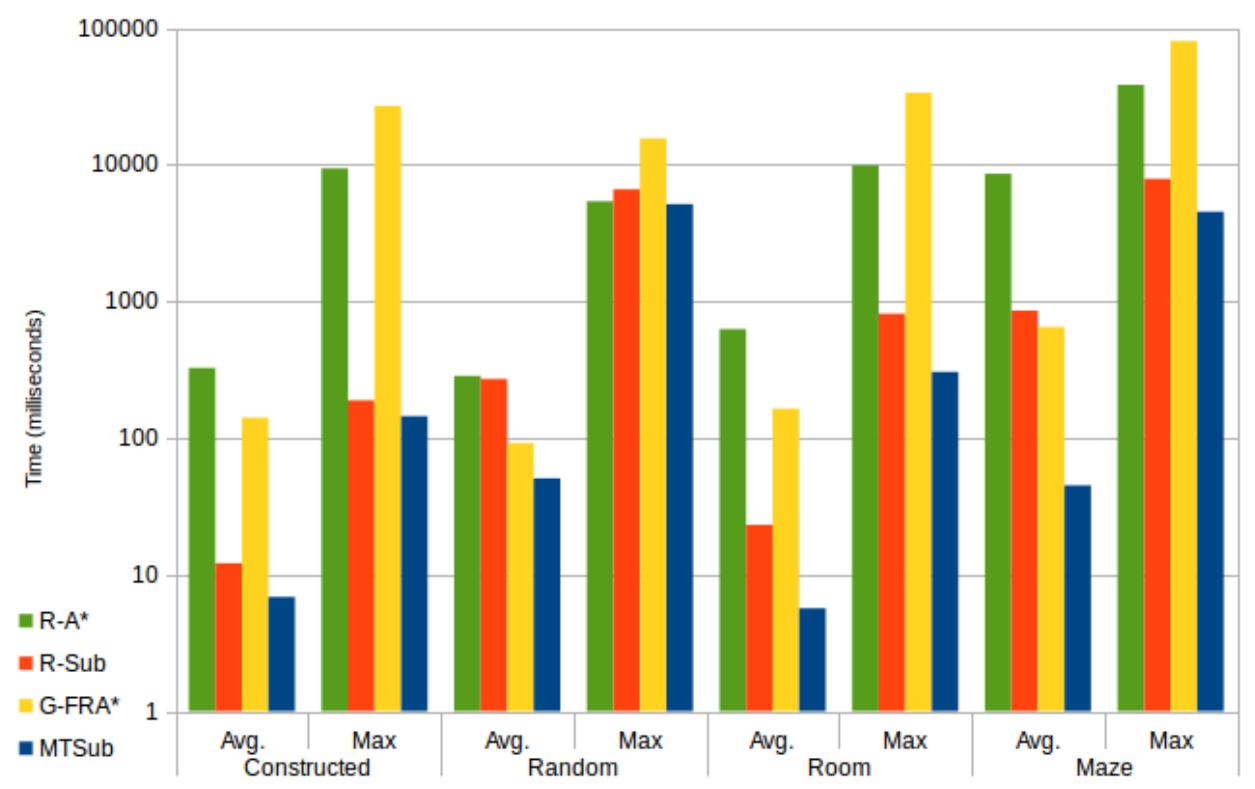

(a) Random Moving Target

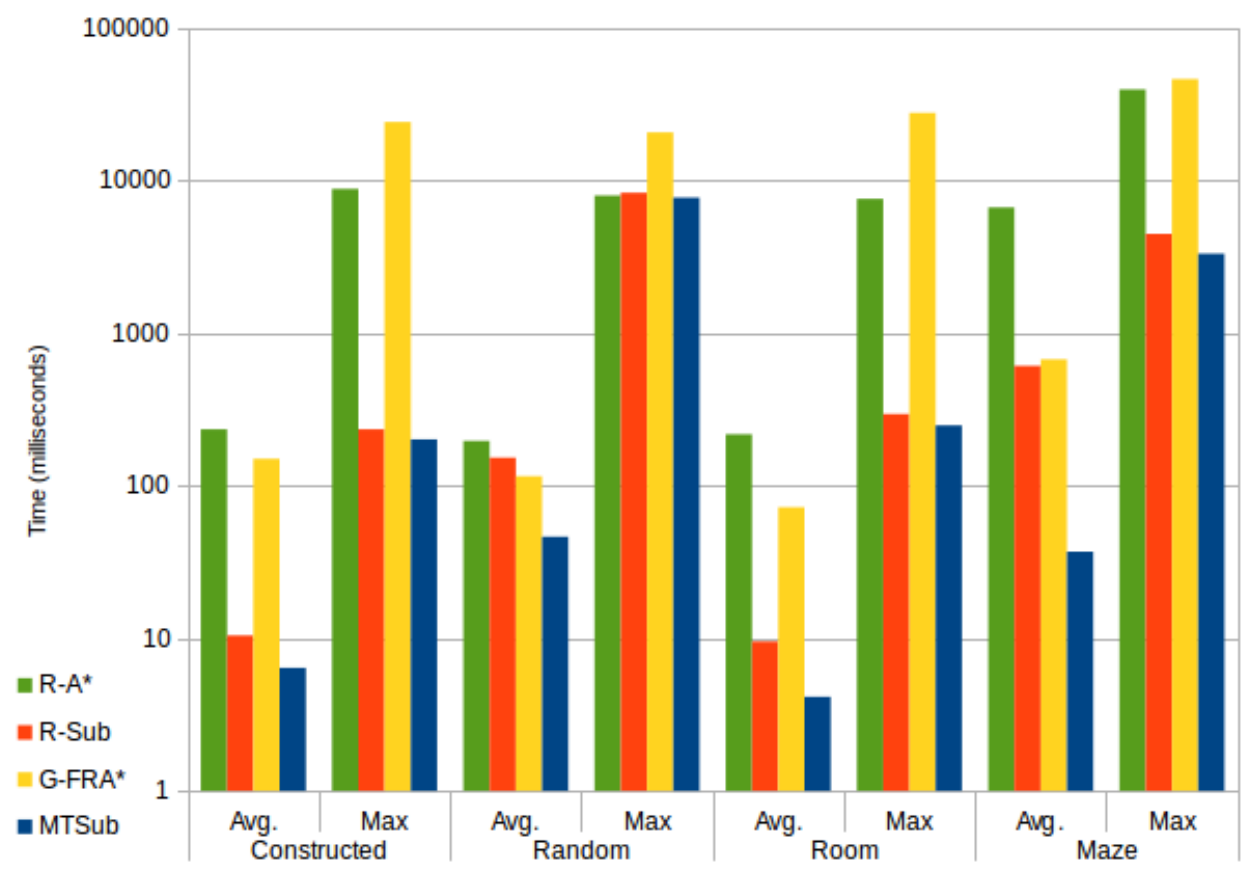

(b) Way-Point Following Target

Figure 4.2: Experiments in 512x512 maps: Average and maximum run times are given for each algorithm. Note that a logarithmic scale is used. 


\begin{tabular}{cl||ccccc} 
& \multicolumn{2}{c}{ R-A* } & R-Sub & G-FRA* & MTSub & R-A*/MTSub \\
\cline { 2 - 7 } Constructed & Avg. & 325.20 & 12.09 & 140.54 & 6.85 & 47.47 \\
& Max & 9369 & 188 & 26857 & 144 & 65.06 \\
\cline { 2 - 7 } Random & Avg & 284.2 & 270.10 & 91.66 & 50.56 & 5.62 \\
& Max & 5389 & 6588 & 15533 & 5140 & 1.04 \\
\cline { 2 - 7 } Room & Avg. & 626.48 & 23.09 & 163.25 & 5.66 & 110.68 \\
& Max & 9858 & 810 & 33507 & 304 & 32.42 \\
\cline { 2 - 6 } Maze & Avg. & 8571.96 & 852.71 & 648.5 & 44.85 & 191.12 \\
& Max & 38315 & 7871 & 80054 & 4523 & 8.47
\end{tabular}

Table 4.2: Experiments in 512 x 512 Maps (Random Moving Target), Times are Given in $\mu$ seconds. All reported times are per step where Avg. is the average time per step and Max is the maximum time per step. Note the effect of map type on the performance of the algorithms.

runtime. It is important to note that MTSub does not have the same issue and it outperforms R-Sub in both average and maximum run times. This is due to the fact that the suggested algorithm only expands an existing search tree or builds a new search tree from scratch as needed.

R-Sub outperforms $\mathrm{A}^{*}$ in both maximum and average times in all of the map classes except Random Maps. The environment abstraction technique used by R-Sub cannot generate significantly smaller abstract graphs for Random Maps. Therefore, R-Sub cannot outperform $A^{*}$ remarkably in such environments. This indicates that environment abstraction can be used to decrease not only average times but also maximum times when the technique can be applied to the environment effectively.

The incremental algorithm G-FRA* has smaller average times than that of RSub not only in Random Maps but also in Maze Maps. This implies that incremental search is comparatively more effective in Maze Maps and Room Maps. This is because the target often does not move to a location that causes G-FRA* to repair its search 
tree.

MTSub outperforms all the competing algorithms in both average and maximum times (for this set of experiments). MTSub uses both environment abstraction and incremental search and successfully avoids the disadvantages of those techniques.

The results also show that the run time performance of MTSub is closely related to the combined success of the techniques it implements. Since R-A* does not use incremental search or environment abstraction, R-A* performance is not affected by how well these techniques work. We used the ratio between R-A* and MTSub time to examine how the success of environment abstraction and incremental search affects MTSub throughout the map classes. The ratio for this set of the experiments can be seen in Table 4.2. Maze and Room Maps have the highest ratios because both environment abstraction and incremental search is very effective in these kinds of maps. The Random Maps have the lowest ratio because Subgoal Graphs are not significantly smaller than the original graph in this kind of maps and therefore the target movement causes more updates in the search tree since the graph is large. Constructed Maps have a ratio that is in between since Constructed Maps have smaller Subgoal Graphs than Random Maps and the target movement causes fewer updates in the existing search tree.

The results of the experiments comparing MTSub against dynamic MTS algorithms with a way-point following target, are depicted in Table 4.3.

According to these results, the use of a way-point following strategy by a target decreases performance of incremental algorithms, namely G-FRA* and MTSub. For instance, G-FRA* no longer has a better average time in Maze Maps than R-Sub 


\begin{tabular}{|c|c|c|c|c|c|c|}
\hline \multirow{3}{*}{ Constructed } & & $\mathrm{R}-\mathrm{A}^{*}$ & R-Sub & G-FRA* & MTSub & R-A*/MTSub \\
\hline & Avg. & 234.26 & 10.42 & 149.63 & 6.39 & 36.66 \\
\hline & Max & 8789 & 234 & 24126 & 201 & 43.72 \\
\hline \multirow{2}{*}{ Random } & Avg & 195.96 & 152.17 & 114.96 & 46.18 & 4.24 \\
\hline & Max & 7952 & 8296 & 20646 & 7715 & 1.03 \\
\hline \multirow{2}{*}{ Room } & Avg. & 216.9 & 9.5 & 72.16 & 4.13 & 52.51 \\
\hline & Max & 7571 & 294 & 27669 & 248 & 30.52 \\
\hline \multirow[t]{2}{*}{ Maze } & Avg. & 6644.91 & 609.23 & 671.73 & $\begin{array}{c}36.85 \\
2300\end{array}$ & 180.32 \\
\hline & $\operatorname{Max}$ & 42 & 4455 & 46099 & 3309 & 11.91 \\
\hline
\end{tabular}

Table 4.3: Experiments in 512 x 512 Maps (Way-point Following Target), Times are Given in microseconds $(\mu)$. All reported times are per step where Avg. is the average time per step and Max is the maximum time per step. Note the effect of target movement strategy on the performance of the algorithms.

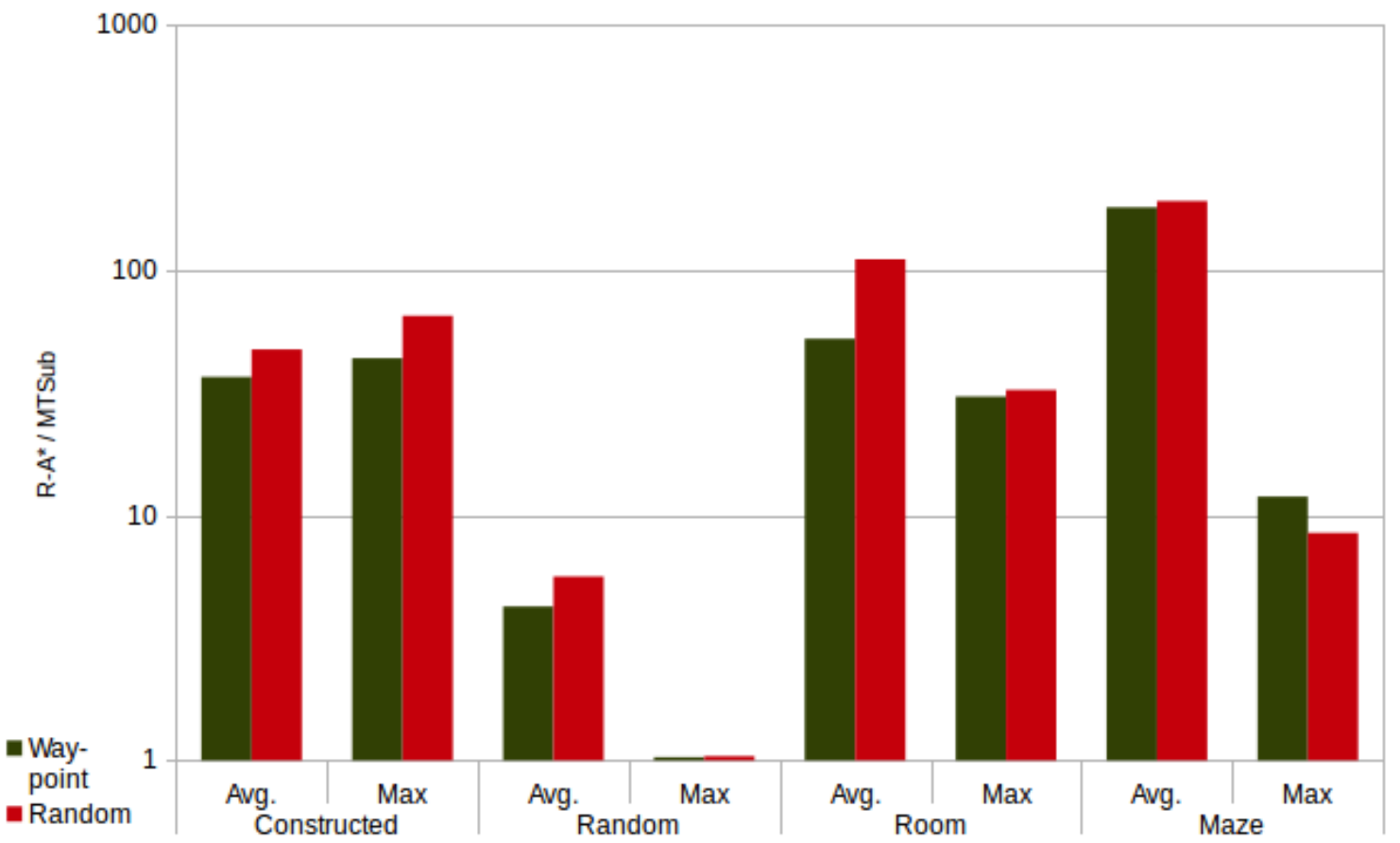

Figure 4.3: Effects of Target Movement Strategy on MTSub: A ratio between R-A* and MTSub is presented. Note that the scale is logarithmic. 
and the ratios of R-A* and MTSub average times is lower in all sections (see Figure 4.3). This is simply because way-point following strategies the target move on a cost minimal path between two points across the map. As a result, the previous plan is updated more often than when a random movement strategy is used.

MTSub outperforms all competing algorithms (in this set of the experiments) even in the cases where an incremental search is less effective with a way-point following target.

We have also examined search times individually in a Constructed Map AR0700SR to complement the experimental results. Below Figure 4.4 shows run times per search of MTSub and R-Sub in a scenario for the AR0700SR map. Search times are given per time $t$. In below Figure 4.4a, we compare MTSub and R-Sub with a randomly moving target. It is shown that after the first time step, MTSub finds paths faster than a repeating algorithm that also uses Two-Level Subgoal Graphs for searching. This implies that incremental search effectively decreases the response time of the agent. In below Figure 4.4b, we compare MTSub and R-Sub with a way-point following target. It is observed that MTSub performs better than R-Sub even if the target's movement strategy forces MTSub to make more computations to update the path. In both graphs, the algorithms spend less time as they come closer to the target.

In both graphs (Figures $4.4 \mathrm{a}$ and $4.4 \mathrm{~b}$ below) we observed spikes in the runtimes of MTSub. The spikes in the graphs occur when the target movement force MTSub to calculate a new search tree. When the target moves randomly, it usually stays in its local area an as such, the existing search path can be reused many times. This results in a few spikes in the graph (Figure 4.4a). Spikes in Figure 4.4b observed more 


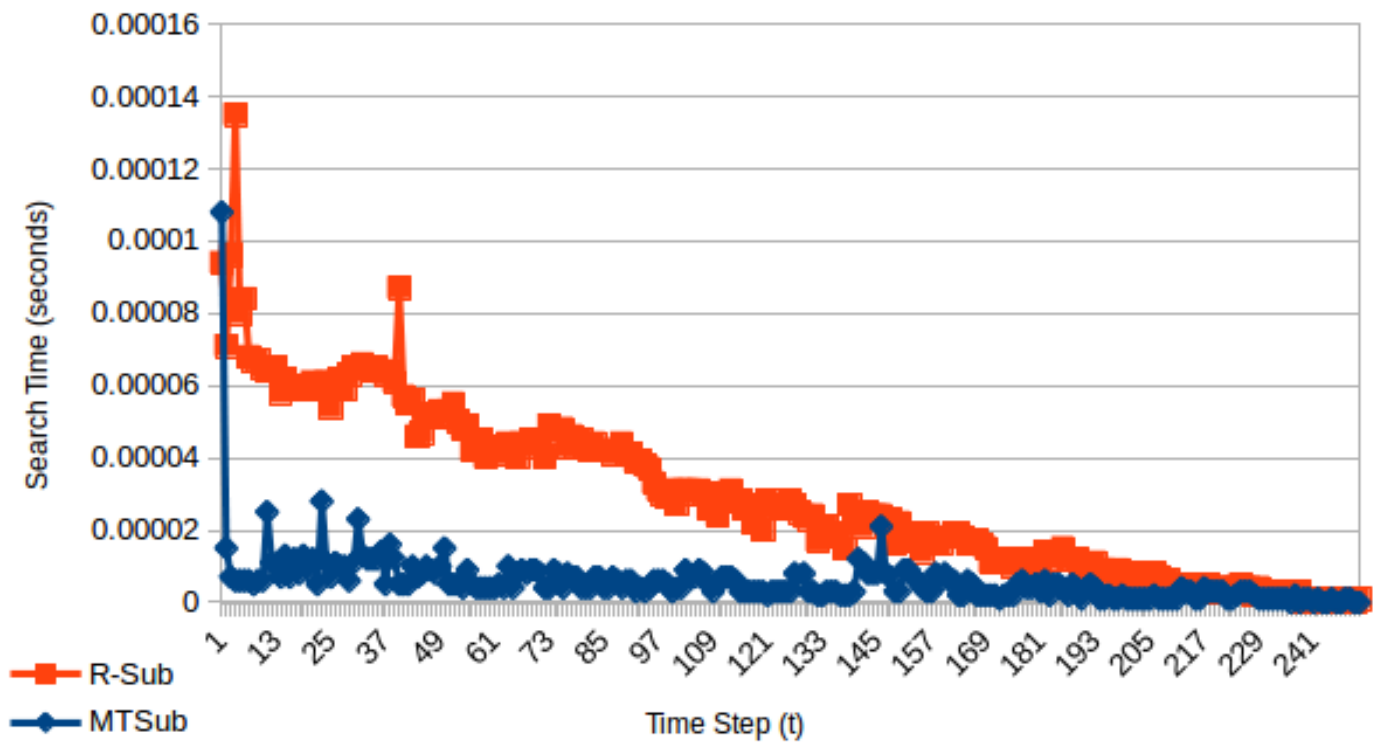

(a) Randomly Moving Target

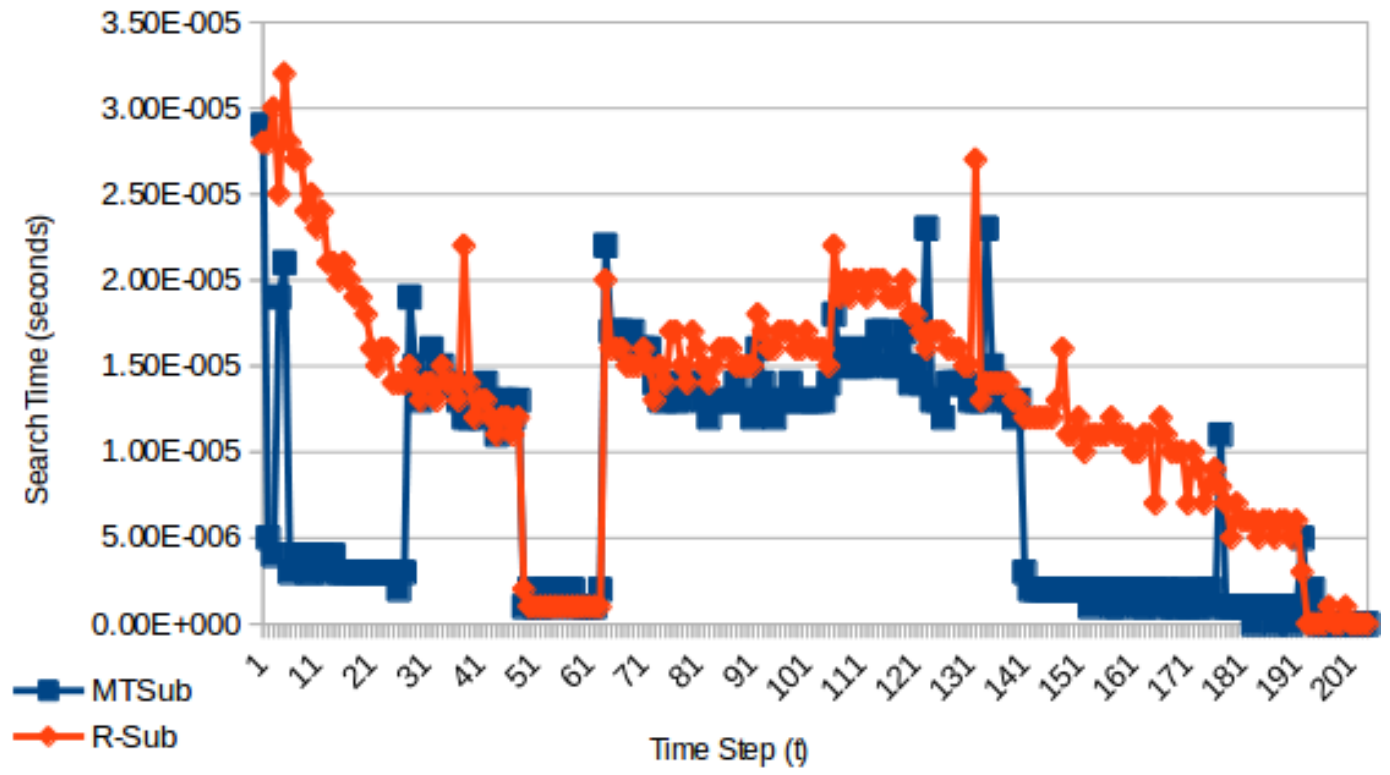

(b) Way-Point Following Target

Figure 4.4: Run Times per Search for MTSub and R-Sub in Map AR0700SR 
often since a way-point following target is more likely to move out of its local area and force MTSub to calculate a new search tree. MTSub and R-Sub have similar times between $t=51$ and $t=71$ in Figure $4.4 \mathrm{~b}$ because they use the same early termination technique, namely quickpath.

\subsubsection{Comparison with MTSCopa}

\begin{tabular}{|c|c|c|c|c|c|c|}
\hline & \multicolumn{2}{|c|}{ Map AR0700SR } & \multicolumn{2}{|c|}{ Map AR0500SR } & \multicolumn{2}{|c|}{ Map AR0300SR } \\
\hline & MTSub & MtsCopa & MTSub & MtsCopa & MTSub & MtsCopa \\
\hline Preprocessing & $0.18 \mathrm{~s}$ & $41299.2 \mathrm{~s}$ & $0.37 \mathrm{~s}$ & $12046 \mathrm{~s}$ & $0.5 \mathrm{~s}$ & $10489.2 \mathrm{~s}$ \\
\hline Memory & $545 \mathrm{~KB}$ & $24064 \mathrm{~KB}$ & $501 \mathrm{~KB}$ & $7680 \mathrm{~KB}$ & $487 \mathrm{~KB}$ & $4403 \mathrm{~KB}$ \\
\hline \multicolumn{7}{|l|}{ Random Move } \\
\hline Average & $6.93 \mu$ & $1.94 \mu$ & $\overline{77.75 \mu}$ & $1.99 \mu$ & $6.61 \mu$ & $1.75 \mu$ \\
\hline Maximum & $184 \mu$ & $27 \mu$ & $149 \mu$ & $84 \mu$ & $147 \mu$ & $25 \mu$ \\
\hline \multicolumn{7}{|l|}{ Waypoint Follow } \\
\hline Average & $\overline{5.68 \mu}$ & $\overline{\overline{1.9 \mu}}$ & 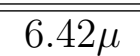 & $\bar{~} 1.42 \mu$ & 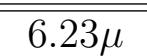 & 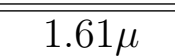 \\
\hline Maximum & $265 \mu$ & $24 \mu$ & $150 \mu$ & $25 \mu$ & $117 \mu$ & $28 \mu$ \\
\hline
\end{tabular}

Table 4.4: Comparison between MtsCopa and MTSub, with respect to preprocessing time and space, and performance. The Experiments were conducted on $320 \times 320$ Maps with a randomly moving target and a way-point following target. Preprocessing shows the preprocessing time in seconds and memory shows the space requirements in $\mathrm{KB}$ after the preprocessing was completed. Average and Maximum are the average time per step and maximum time per step respectively.

This section presents the results of the experiments that compare MTSub against MTSCopa, which in the preprocessing phase computes a path between any two nodes $s, s^{\prime} \in S$ and stores the results in a database. 


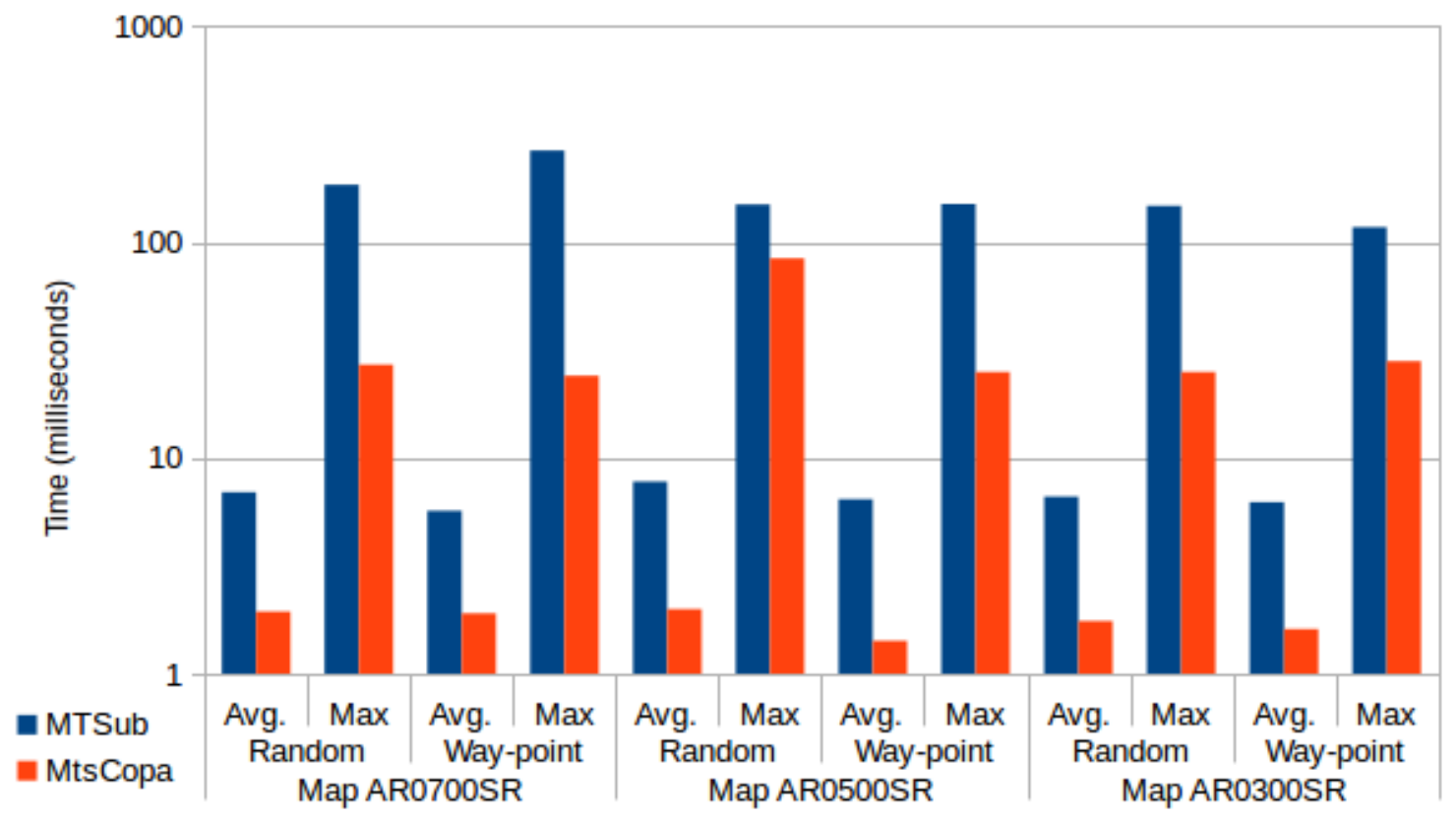

Figure 4.5: Run Time of MTSub and MTSCopa

The results of the experiments comparing MTSub against MTSCopa with a random moving and a way-point following target are shown in Table 4.4, depict preprocessing time and space consumption in addition to the average and maximum search times. Figure 4.5 also visualizes the results.

In our experiments, MTSub outperformed MtsCopa by up to 229400 times in terms of preprocessing time, and up to 44.15 times in terms of space requirements. While significantly better during the preprocessing phase, MTSub did not outperform MTSCopa at runtime. However, our experiments show that MTSub provides competitive runtimes to MTSCopa. In experiments with a random moving target, MtsCopa is better than MTSub by up to only 3.89 times in average search times and up to 6.81 times in maximum times. In experiments with a random moving target, MtsCopa is better than MTSub by only up to 4.52 times in average search times and 
up to 11 times in maximum times. These results confirm our previous observation that way-point movement strategy slightly hurts MTSub performance.

It is important to note that we conducted these experiments with only small sized Constructed Maps due to the significant preprocessing times of MTSCopa. We chose a constructed map because they are better representation of a real life environment than any other type of map. From the experiments we estimated the step time requirements of MTSCopa by using a ratio between the average step time and the path length. Also, analysis of the data from GPPC-2012 ${ }^{1}$ suggests that MTSCopa's ratios of average and maximum times to path length are not significantly affected by the map domain. The large amount of expected MTSCopa preprocessing time of $512 \times 512$ maps (estimated at 30-60 hours per map), and the fact that the performance of MTSCopa should not vary much from map to map, led to the conclusion that it is not necessary to conduct tests on a variety of maps. As discussed in Chapter 2 MTSCopa constructs a database that allows it to determine the next cell to move to (from the starting cell to the target cell) for given start and target cells. Therefore, the performance of MTSCopa is independent of the map domain and is depended on the speed in which the query can be answered. The results in graph 4.6 shows that the MTSCopa run times and we believe that minor differences in run times because of memory caching. Thus, each step of MTSCopa is a constant time regardless of the map domain. The expected performance of MTSub for random maps will not be as good as in the case of a constructed map. The performance degradation would depend on the number of random obstacles that are introduced to the environment. This is due to the direct impact of the size of the subgoal graphs. Here, it is expected

\footnotetext{
${ }^{1}$ http://movingai.com/GPPC/results.html
} 
that the performance of MTSub would be about 25-30 times slower than MTSCopa, which is more significant. On the other hand, MTSub performs better in Room and Maze maps. We expect that MTSub would be about 2-3 times slower than MTSCopa in these map classes.

\begin{tabular}{lccc} 
Map Name & Map Size & $\begin{array}{c}\text { Preprocessing } \\
\text { Time }\end{array}$ & Space \\
\hline \hline Aftershock & $512 \times 512$ & $1989.26 \mathrm{~ms}$ & $1.3 \mathrm{MB}$ \\
Backwoods & $512 \times 768$ & $754.164 \mathrm{~ms}$ & $1.9 \mathrm{MB}$ \\
RiverLethe & $512 \times 768$ & $1685.51 \mathrm{~ms}$ & $1.9 \mathrm{MB}$ \\
Tribes & $768 \times 768$ & $6381.02 \mathrm{~ms}$ & $2.7 \mathrm{MB}$ \\
Turbo & $768 \times 768$ & $1691.91 \mathrm{~ms}$ & $2.6 \mathrm{MB}$ \\
Nightshade & $768 \times 1024$ & $4348.23 \mathrm{~ms}$ & $3.7 \mathrm{MB}$ \\
TheFrozenSea & $1024 \times 1024$ & $7691.85 \mathrm{~ms}$ & $5.4 \mathrm{MB}$ \\
Octopus & $1024 \times 1024$ & $5571.44 \mathrm{~ms}$ & $4.9 \mathrm{MB}$ \\
Cauldron & $1024 \times 1024$ & $5063.05 \mathrm{~ms}$ & $5.2 \mathrm{MB}$
\end{tabular}

Table 4.5: Preprocessing Requirements of MTSub

We have provided the required preprocessing time and space for MTSub in various maps in Table 4.5 to complement the results of our experiments. The results show that MTSub is applicable not only to $512 \times 512$ maps but also to larger maps.

In Figure 4.6 below, we compare search times of MTSub and MtsCopa individually in a Constructed Map AR0700SR. Search times are given per time $t$. We exclude maximum run times of both of the algorithms from graphs to provide a better picture (Both maximum times occurred at $t=0$ in both cases). Both graphs (for a randomly moving target and a waypoint following target) show that the runtime of MTSub has jumps. The jumps occur when the existing search tree is no longer usable and MTSub constructs a search tree from scratch. These jumps do not exceed the maximum 


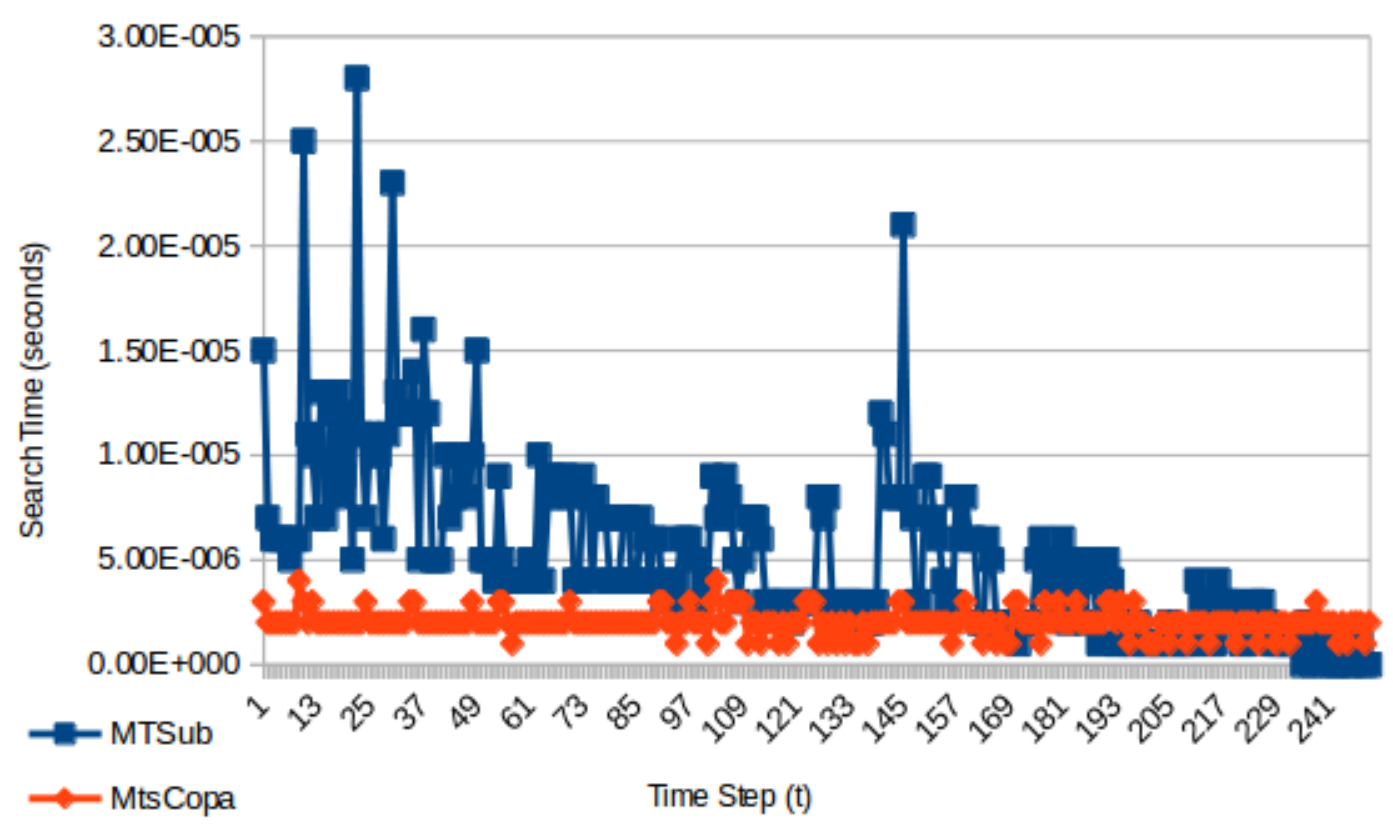

(a) Randomly Moving Target

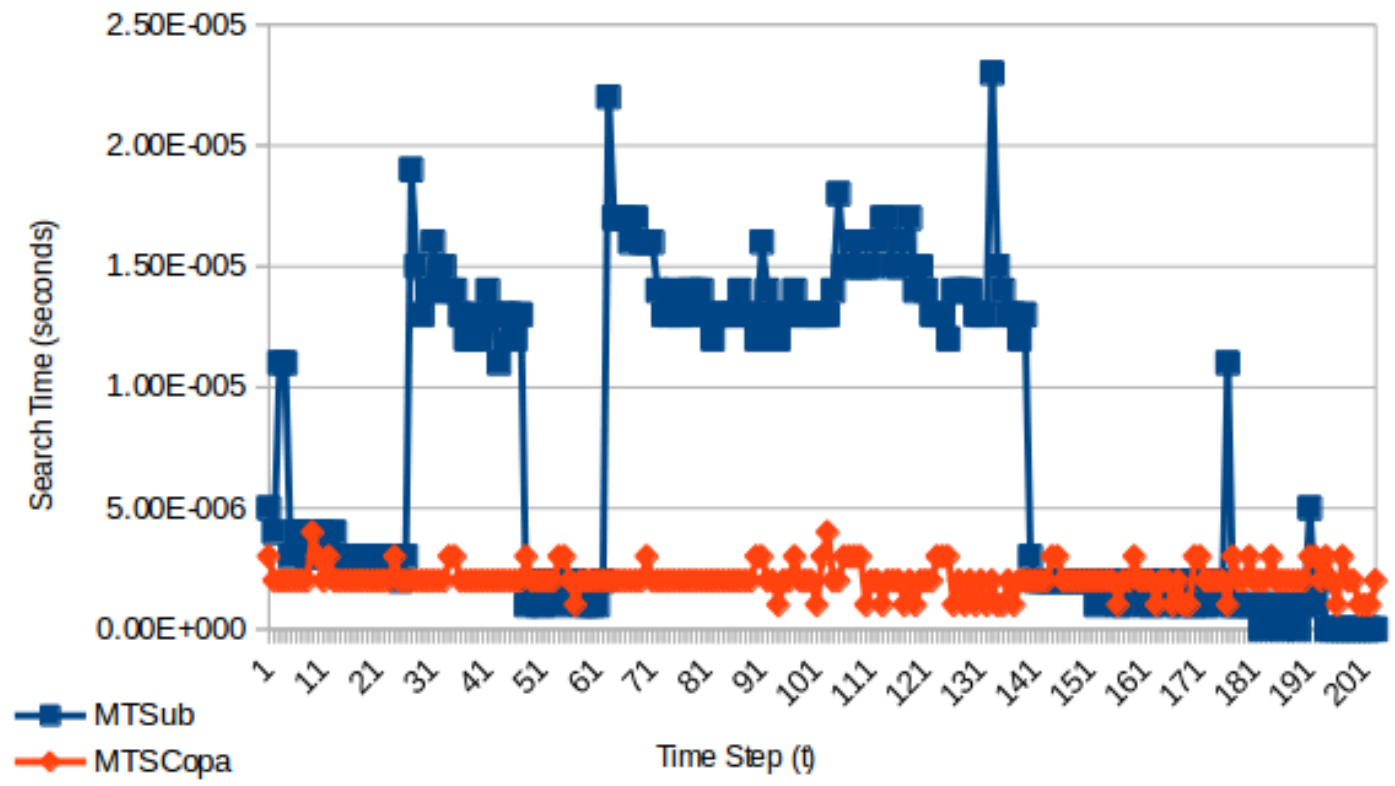

(b) Way-Point Following Target

Figure 4.6: Run Times per Search for MTSub and MTSCopa in Map AR0700SR 
runtime of MTSub and get smaller as the agent gets closer to the target. These kinds of jumps occur more often if a target is following a way-point because the target is more likely to move out of its local area and force updates. However, MtsCopa maintains almost the same runtime after the maximum time without being affected by the distance between the agent and the target, or the movement strategy of the target. This is because MtsCopa implements a look up operation to return a next move instead of returning a complete path and MTSCopa is not an incremental algorithm. This also forces MtsCopa to spend the same amount of time even if the target has not moved between time steps. This implies that the difference between MTSub and MtsCopa decreases as a target skips moves more often.

\subsubsection{Comparison with Extensions}

This section discusses possible extensions of MTSub aimed meeting different constraints and presents the preliminary results of the related experiments. We explain how response time constraints may be met and how it may be possible to use MTSub in different environment representations. This subsection also discusses how the MTSub algorithm can possibly catch a target with less cost than that of an optimal algorithm for MTS.

\section{Working Under Strict Time Limits}

In some applications, such as video games and dynamic robots, a tighter constraint is imposed on the response time. A response within the acceptable time limits is better than a late one even if quality of the resulting response is not optimal. For example, a 
fast moving mobile robot needs fast updates that match its speed to move smoothly.

MTSub can meet real time requirements but there is still room for improvement on response time. This can be achieved with trading space or optimality of the plan for shorter response times:

- Trading Memory: MTSub uses very little memory in preprocessing as seen in Table 4.5. It may be possible to use extra space to speed up response time. MTSub connects $s_{T}$ at every time $t$ to the Two-Level Subgoal Graph. According to our experiments in $512 \times 512$ Constructed Maps, this operation takes up to $21 \%$ of the maximum response time and up to $51 \%$ of the average time. It is possible to save the topologies of Two-Level Subgoal Graph after the possible locations of the target are connected to the graph. This can be done for each possible location of the target, saving edges that connect the target to the graph. We estimate that an improvement of up to $21 \%$ o thef maximum and $51 \%$ of the average time can be achieved with $20 \mathrm{~KB}$ extra space and $120 \mathrm{~ms}$ extra preprocessing time. This estimation depends on experiments in Constructed Maps. The performance of the technique may differ in other map classes. We conjecture that, as the number of unblocked grid cells goes up, space and preprocessing times to implement the method increases.

- Trading Optimality: Anytime Repairing A* (ARA*) [15] and ARA*'s extension for MTS, Incremental Anytime Repairing A* (I-ARA*) [13], are algorithms that sacrifice optimality of the plan to decrease response time. To do so, they inflate heuristic values with a constant value $(e)$ to speed up $\mathrm{A}^{*}$ search. According to the Theorem 1 in [15], an $\mathrm{A}^{*}$ search with an inflated heuristic value, $h(s)=$ 
$H\left(s, s_{T}\right) * e$ where $s \in S$, finds a path $\Pi$ where $C\left(s_{A}, s_{T}\right) \leq C\left(\Pi\left(s_{A}, s_{T}\right)\right) \leq$ $C\left(s_{A}, s_{T}\right) * e$. Our preliminary results with the value $e=2.5$ in Constructed Maps show that an improvement of $11 \%$ in average times and $22 \%$ in maximum times can be achieved at the expense of $36 \%$ additional cost of catching a target.

\section{Decreasing The Cost of Reaching A Target}

In some applications, more computation time is available to the agent during a search. This additional time can be used to estimate future locations of the target, which may lead to catching the target with less cost.

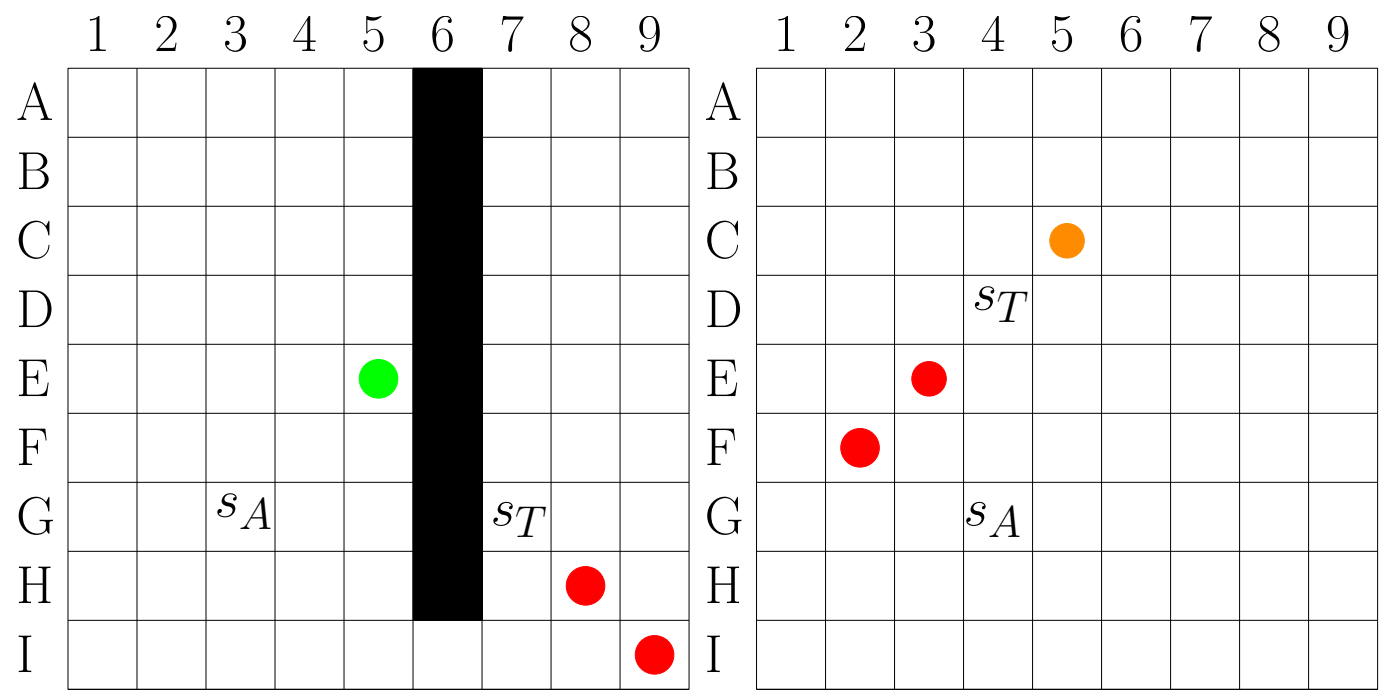

(a) Meeting Point is Found

(b) Meeting Point is not Found

Figure 4.7: Moving Window Example: Green dot represents the estimated meeting point. Red dots indicate past locations of the target. Yellow dot represents the future location of the target, which is the aim of the agent when calculating a meeting point is not possible.

We used a simple method to observe this technique's effect on the cost of reaching a target. First, we calculate a vector for a target by observing its past $k$ moves. We 
then calculate if it is possible to determine a meeting location with the target. Since the agent and the target move at the same speed, such a point exists if the target's movement vector makes a smaller angle than 90 with the vector between locations of the agent and the target. If a meeting location can be found, the agent goes to the meeting point. Otherwise, the agent follows the target's future location, which is $n$ time steps ahead of the current time step (if the agent can reach the target sooner than $n$ time steps, the agent goes directly to the target location.). We call this estimation method "Moving Window". The best values of $k$ and $n$ are specific to the environment and they may change during a search. An example of Moving Window can be seen in Figure 4.7. We use $k=2$ and $n=1$ for the example. In Figure 4.7a, since the target's movement vector makes a smaller angle than 90 with the vector between locations of the agent and the target, Moving Window finds a meeting point (green dot). Note that the estimation assumes that the agent and the target move in a free environment. In Figure 4.7b, it is not possible to calculate a meeting point since the angle is bigger than 90 degrees. The agent follows position $\left(T, t_{i+n}\right)$ (yellow dot).

\begin{tabular}{cccc} 
& MTSub & MTSub with Prediction & Improvement (\%) \\
\hline Random Maps & 181662 & 159701 & 13.75 \\
Constructed Maps & 277032 & 257622 & 7.53 \\
Maze Maps & 927936 & 1156361 & -19.75 \\
Room Maps & 375864 & 395557 & -4.97
\end{tabular}

Table 4.6: MTSub vs. MTSub with Moving Window: The amount of time required to catch a target given as time step $(t)$ for each competing algorithm. Improvement of the extension algorithm is indicated by its ratio to MTSub.

The results of the experiments that examine the effectiveness of target movement prediction are shown in Table 4.6. We present the best results that we were able to 
accomplish, achieved with the values, $k=7$ and $n=5$.

The cost of reaching a target is increased in Maze and Room Maps. This is because we estimate the future location the target as if there are no obstacles in the environment and the actual distance between the estimated and actual future locations of the target is high in these kinds of maps. In Random and Constructed Maps, where the correctness of the estimations are higher, MTSub with Prediction decreases the cost of reaching a target.

\section{Operating in Different Environment Representations}

MTSub operates in only 8 connected grid environments since the environment abstraction method that is used requires it. However, it might be possible to use the concepts proposed in MTSub with different environment abstraction techniques to work in other environment representations.

A generic algorithm for MTS with environment abstraction is given in Algorithm 4.1. This is to emphasize the requirements of algorithms like MTSub that are dictated by the environment abstraction. Incremental methods of MTSub can be directly implemented by other abstraction methods. The update of the abstraction graph (line 4) must be quick since it is a runtime operation. The improvement of the average time depends on this while we expect maximum times of the algorithms will be the same as that of the offline use of abstraction techniques. Visibility Graphs [11] and Navigation Meshes may be promising for this extension idea. 


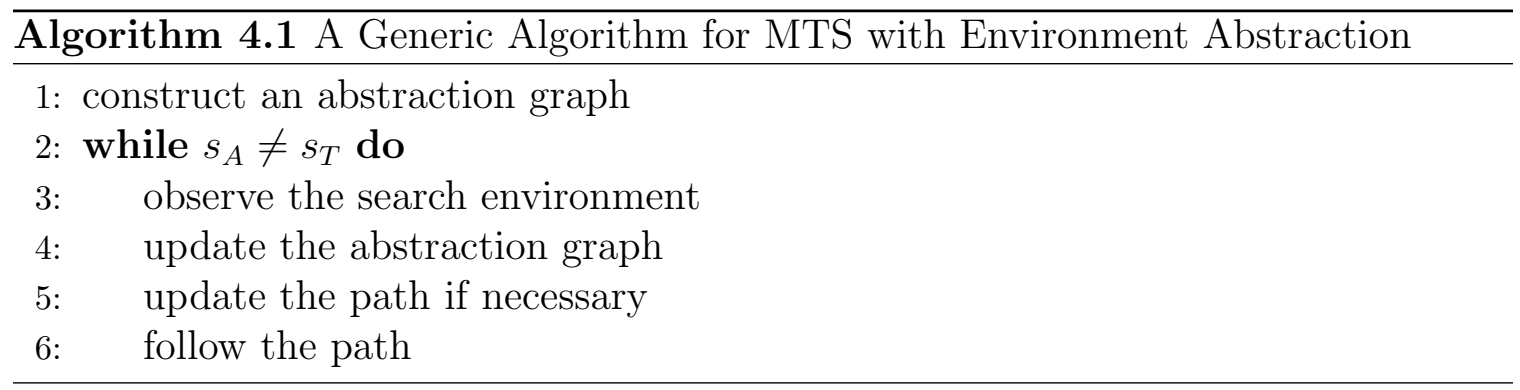

\subsection{Summary}

This chapter presented an empirical evaluation of MTSub and its variations.

In section 4.1, the objective of the experiments was to measure success of the suggested algorithms according to the criteria discussed in section 3.1. We explained that MTSub is compared against two algorithms for MTS. Namely, G-FRA*, which is an incremental algorithm, and MtsCopa which is the state-of-art algorithm where required preprocessing time and memory are available. MTSub is also compared with algorithms for fixed location path planning to demonstrate the benefits of incremental search ( $\mathrm{A}^{*}$ and Two-Level Subgoal Graphs).

Compared to G-FRA*, which is the best known dynamic algorithm so far, MTSub is up to 29 times faster in average time per step, and 186 times faster in maximum time per step. MTSub outperforms all competing dynamic algorithms even if incremental search is less effective with a way-point following target.

MTSub also compares fairly well against MtsCopa. Although in this case MTSub is up to 3.89 times slower in average response time and up to 6.81 times slower in maximum response time, it performed much better than MtsCopa in the processing phase - up to 220,000 times faster and requiring up to 44 times less space. 
The preliminary results show that suggested extensions of MTSub may be beneficial to meet the constraints of different applications. 


\section{Chapter 5}

\section{Conclusion}

Moving Target Search (MTS) is a path planning problem where an agent attempts to reach a moving target. In this regard, the agent and the target move at discrete time steps and at each step the agent can obtain the target's updated location. The objective of the agent is to reach the target while minimizing the cost of its route. In this work, we focus on moving target search where the agent has full knowledge of the search environment, which is static. This variant of MTS often arises in robotic applications that run in a known environment and in video games.

This thesis introduces an innovative algorithm for MTS, namely MTSub. MTSub uses incremental search and environment abstraction to decrease calculation time of a route. Empirical evaluation showed that the MTSub algorithm outperforms the competing dynamic online algorithms, G-FRA*, R-Sub and R-A*, in all the experiments. Empirical analysis also showed that MTSub, when compared against MTSCopa, which is a state-of-the-art algorithm for MTS, has a significantly smaller 
memory footprint and requires less preprocessing time and while slower, still has competitive average and maximum response times. Although slower than MtsCopa, which relies heavily on preprocessing, based on the experiment results, MTSub establishes itself as the new state-of-the-art in optimal moving target search without heavy-weight preprocessing. Experimental results show that MTSub can meet real time performance requirements (e.g., 5 microseconds per step). Compared to the best known dynamic algorithm to date, MTSub is up to 29 times faster in average time per step and 186 times faster in maximum time per step. MTSub also compares fairly well against an algorithm that requires heavy pre-processing. Although in this case MTSub is up to 3.89 times slower in average response time and up to 6.81 times slower in maximum response time, it performed much better than MTSCopa in the preprocessing phase - up to 220,000 times faster and requiring up to 44 times less space.

MTSub's novelty is in combining incremental search and environment abstraction techniques. This allows MTSub to reduce the number of times it needs to compute the cost minimal path between the agent and the target. Our empirical analysis showed that MTSub faces performance degradation in Random Map domains since Subgoal Graph is not much smaller than the original graph. MTSub performs best in the Room Map domain since the environment abstraction technique (Subgoal Graphs) is particularly good in that domain. The experiments also showed that the target movement strategy has an effect on the performance of MTSub. This is because the target movements determine the effectiveness of the incremental search. A way-point following target is more likely to move out of its local area and force MTSub to find a path from scratch. However, a target that moves random is more likely to stay in 
its local area and incremental search is more effective. MTSub performs better with random movers for those reasons. Even if MTSub performance varies according to the target movement strategy and the map domains, it still outperforms competing algorithms as mentioned previously.

The low response time of MTSub in comparison to the other algorithms makes MTSub an ideal algorithm for gaming and robotics applications. This implies that dynamic robotic and video game applications that have to operate in large search spaces can obtain optimal solutions for the Moving Target Search problem in realtime. For instance, using MTSub a policeman in a video game can catch a robber and the policeman motion can be viewed smoothly. This is because MTSub enables high frame rate.

In future work, we plan to adapt our algorithm to dynamic environments. Another research direction is to relax the optimality criteria and explore the impact of realtime constraints on the performance and quality of the generated path. 


\section{Bibliography}

[1] E. W. Dijkstra, "A note on two problems in connexion with graphs," Numerische mathematik, vol. 1, no. 1, pp. 269-271, 1959.

[2] P. E. Hart, N. J. Nilsson, and B. Raphael, "A formal basis for the heuristic determination of minimum cost paths," Systems Science and Cybernetics, IEEE Transactions on, vol. 4, no. 2, pp. 100-107, 1968.

[3] D. Ferguson and A. Stentz, "Field d*: An interpolation-based path planner and replanner," in Robotics Research, pp. 239-253, Springer, 2007.

[4] T. Ishida and R. E. Korf, "Moving target search.," in International Joint Conference on Artificial Intelligence (IJCAI), vol. 91, pp. 204-210, 1991.

[5] T. Ishida and R. E. Korf, "Moving-target search: A real-time search for changing goals," Pattern Analysis and Machine Intelligence, IEEE Transactions on, vol. 17, no. 6, pp. 609-619, 1995.

[6] C. Moldenhauer and N. R. Sturtevant, "Optimal solutions for moving target search," in Proceedings of The 8th International Conference on Autonomous 
Agents and Multiagent Systems-Volume 2, pp. 1249-1250, International Foundation for Autonomous Agents and Multiagent Systems, 2009.

[7] X. Sun, W. Yeoh, and S. Koenig, "Efficient incremental search for moving target search," in Twenty-First International Joint Conference on Artificial Intelligence, pp. 615-620, 2009.

[8] X. Sun, S. Koenig, and W. Yeoh, "Generalized adaptive a*," in Proceedings of the 7th international joint conference on Autonomous agents and multiagent systems-Volume 1, pp. 469-476, International Foundation for Autonomous Agents and Multiagent Systems, 2008.

[9] A. Botea, J. A. Baier, D. Harabor, and C. Hernández, "Moving target search with compressed path databases.," in International Conference of Automated Planning and Scheduling (ICAPS), pp. 288-292, 2013.

[10] V. Bulitko, Y. Björnsson, M. Lustrek, J. Schaeffer, and S. Sigmundarson, "Dynamic control in path-planning with real-time heuristic search.," in International Conference of Automated Planning and Scheduling (ICAPS), pp. 49-56, 2007.

[11] T. Lozano-Pérez and M. A. Wesley, "An algorithm for planning collision-free paths among polyhedral obstacles," Communications of the ACM, vol. 22, no. 10, pp. $560-570,1979$.

[12] X. Sun, W. Yeoh, and S. Koenig, "Moving target d* lite," in Proceedings of the 9th International Conference on Autonomous Agents and Multiagent Systems: volume 1-Volume 1, pp. 67-74, International Foundation for Autonomous Agents and Multiagent Systems, 2010. 
[13] X. Sun, W. Yeoh, T. Uras, and S. Koenig, "Incremental ara*: An incremental anytime search algorithm for moving-target search," in Twenty-Second International Conference on Automated Planning and Scheduling, pp. 243-232, 2012.

[14] C. Hernández, J. A. Baier, T. Uras, and S. Koenig, "Position paper: Incremental search algorithms considered poorly understood.," in International Annual Symposium on Combinatorial Search (SOCS), pp. 82-86.

[15] M. Likhachev, G. J. Gordon, and S. Thrun, "Ara*: Anytime a* with provable bounds on sub-optimality," in Advances in Neural Information Processing Systems, pp. 310-318, 2003.

[16] A. Patel, "Amits thoughts on pathfinding," 2015. http://theory.stanford.edu/ amitp/GameProgramming.html, visited 2015$02-11$.

[17] X. Cui and H. Shi, "A*-based pathfinding in modern computer games," International Journal of Computer Science and Network Security, vol. 11, no. 1, pp. 125-130, 2011.

[18] G. Hahn and G. MacGillivray, "A note on k-cop, l-robber games on graphs," Discrete mathematics, vol. 306, no. 19, pp. 2492-2497, 2006.

[19] M. A. Vieira, R. Govindan, and G. S. Sukhatme, "Optimal policy in discrete pursuit-evasion games," Department of Computer Science, University of Southern California, Tech. Rep, pp. 08-900, 2008. 
[20] M. A. Vieira, R. Govindan, and G. S. Sukhatme, "Scalable and practical pursuitevasion with networked robots," Intelligent Service Robotics, vol. 2, no. 4, pp. 247-263, 2009.

[21] B. Strasser, D. Harabor, and A. Botea, "Fast first-move queries through runlength encoding," in Seventh Annual Symposium on Combinatorial Search, pp. $157-165,2014$.

[22] S. Koenig and M. Likhachev, "Fast replanning for navigation in unknown terrain," Robotics, IEEE Transactions on, vol. 21, no. 3, pp. 354-363, 2005.

[23] X. Sun, W. Yeoh, and S. Koenig, "Generalized fringe-retrieving a*: faster moving target search on state lattices," in Proceedings of the 9th International Conference on Autonomous Agents and Multiagent Systems: volume 1-Volume 1, pp. 10811088, International Foundation for Autonomous Agents and Multiagent Systems, 2010.

[24] A. Botea, "Ultra-fast optimal pathfinding without runtime search.," in The Artificial Intelligence for Interactive Digital Entertainment Conference, pp. 123-127, 2011.

[25] J. Baier, A. Botea, D. Harabor, and C. Hernandez, "A fast algorithm for catching a prey quickly in known and partially known game maps," Computational Intelligence and AI in Games, IEEE Transactions on , vol.PP, no.99, pp. 1-1.

[26] T. Uras, S. Koenig, and C. Hernández, "Subgoal graphs for optimal pathfinding in eight-neighbor grids," in Twenty-Third International Conference on Automated Planning and Scheduling, pp. 224-232, 2013. 
[27] T. Uras and S. Koenig, "Identifying hierarchies for fast optimal search," in Seventh Annual Symposium on Combinatorial Search, pp. 878-884, 2014.

[28] S. Koenig, M. Likhachev, and X. Sun, "Speeding up moving-target search," in Proceedings of the 6th international joint conference on Autonomous agents and multiagent systems, p. 188, ACM, 2007.

[29] S. Koenig and M. Likhachev, "D* lite.," in AAAI/Innovative Applications of Artificial Intelligence Conference (IAAI), pp. 476-483, 2002.

[30] GPPC, "Grid based path-planning competition," 2015. http://movingai.com/GPPC/, visited 2015-02-11.

[31] HOG, "Hog2 library," 2015. https://code.google.com/p/hog2/, visited 2015-0211.

[32] N. R. Sturtevant, "Benchmarks for grid-based pathfinding," Computational Intelligence and AI in Games, IEEE Transactions on, vol. 4, no. 2, pp. 144-148, 2012. 
Appendices 


\section{Appendix A}

\section{Glossary}

$V: V$ represents all the grid cells.

$E$ : $E$ corresponds to allowable moves between neighbouring grid cells.

blocked(obstacle) / unblocked(free) cell: The blocked or obstacle cells are the cells which the agents can not traverse and sit on where as the unblocked or free cells are the grid cells which the agents can traverse and sit on.

$G=(V, E):$ An undirected graph $G=(V, E)$ represents the environment. The nodes can be either blocked or unblocked. The environment is static, which implies that nodes do not change their status over time (e.g., becoming an obstacle or ceasing being an obstacle).

$V_{s}: V_{s}$ represents all the subgoal nodes.

$E_{s}: E$ corresponds to allowable moves between neighbouring subgoals.

$G_{s}=\left(V_{s}, E_{s}\right)$ : An undirected graph $G_{s}=\left(V_{s}, E_{s}\right)$ represents a Subgoal Graph. 
$S$ : $S$ denotes all the unblocked nodes in $V$, where $S \subset V$.

$C\left(s, s^{\prime}\right)$ : We denote by $C\left(s, s^{\prime}\right)$ the smallest cost of travelling from $s$ to $s^{\prime}$ where $\left(s, s^{\prime}\right) \in S$.

neighbour $(s)$ : The notation neighbour $(s)$ denotes the set of all nodes $s^{\prime} \in S$ that $s$ can move to by an allowed move.

sg.neighbour(s): All nodes in a Subgoal Graph that is connected to $s$ with an edge.

$\Pi\left(s, s^{\prime}\right)$ : An ordered set of adjacent nodes that form a path from $s$ to $s^{\prime}$ where $s, s^{\prime} \in S$.

$C\left(\Pi\left(s, s^{\prime}\right)\right.$ : We define $C\left(\Pi\left(s, s^{\prime}\right)\right)$ as cost of the path $\Pi$.

$t$ : We use $t$ to indicate discrete time steps from the beginning of the search until the end of the search.

$\operatorname{position}\left(A, t_{i}\right)$ and position $\left(T, t_{i}\right)$ : Notations $\operatorname{position}\left(A, t_{i}\right)$ and $\operatorname{position}\left(T, t_{i}\right)$ are used to show locations of the agent and target at time $t_{i}$, respectively.

$s_{A}$ and $s_{T}$ : For simplicity we also use $s_{A}$ and $s_{T}$ to indicate the location of the agent and the target at the current time, respectively. They indicate initial positions of $A$ and $T$ before the initialization of a search.

route: We use route to denote an ordered collection of position $(A, t), t_{0} \leq t \leq t_{k}$, where $t_{0}$ is the time that the agent started to move towards the target and $t_{k}$ is the time that the agent reached the moving target.

$h(s)$ The approximation cost - used to denote the user provided approximation of $C\left(s, s_{T}\right)$ where $s, s_{T} \in S$. 
$H\left(s, s_{T}\right)$ : Represents the function used to compute $h(s)$.

$g(s)$ Calculated value for $C\left(s_{A}, s\right)$ - this value is denoted $g(s)$.

$f(s)$ : Hierarchical cost of reaching the target, where $f(s)=g(s)+h(s)$.

search tree: A tree data structure over a graph that is constructed by a search algorithm.

parent $(s)$ : Denotes the node that is the parent of $s$ in the search tree.

$O P E N$ : This list maintains the nodes that are going to be evaluated by $\mathrm{A}^{*}$.

CLOSED: This list contains all of the nodes that were evaluated (expanded) by A*.

way-point: A designated unblocked grid cell on the route of the target. 